\title{
DEPLETION MODELING OF LIQUID DOMINATED GEOTHERMAL RESERVOIRS
}

Gudmund Olsen

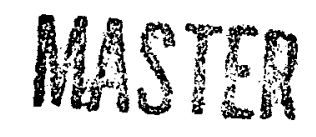

\footnotetext{
OT MICROFILM

COVER

June 1984
}

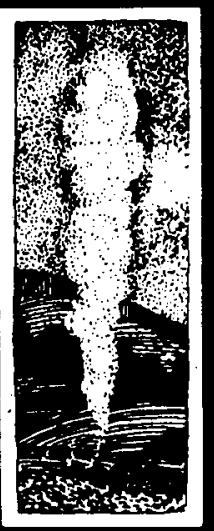

Stanford Geothermal Program INTERDISCIPLINARY RESEARCH IN ENGINEERING AND EARTH SCIENCES Stanford University, Stanford, California 


\section{DISCLAIMER}

This report was prepared as an account of work sponsored by an agency of the United States Government. Neither the United States Government nor any agency Thereof, nor any of their employees, makes any warranty, express or implied, or assumes any legal liability or responsibility for the accuracy, completeness, or usefulness of any information, apparatus, product, or process disclosed, or represents that its use would not infringe privately owned rights. Reference herein to any specific commercial product, process, or service by trade name, trademark, manufacturer, or otherwise does not necessarily constitute or imply its endorsement, recommendation, or favoring by the United States Government or any agency thereof. The views and opinions of authors expressed herein do not necessarily state or reflect those of the United States Government or any agency thereof. 


\section{DISCLAIMER}

Portions of this document may be illegible in electronic image products. Images are produced from the best available original document. 


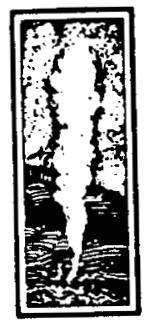

\author{
Stanford Geothermal Program \\ Interdisciplinary Research in \\ Engineering and Earth Sciences \\ STANFORD UNIVERSITY \\ Stanford, California
}

SGP-TR- -80

DE85 009923

SGP-TR-80

DEPLETION MODELING OF LIQUID DOMINATED GEOTHERMAL RESERVOIRS

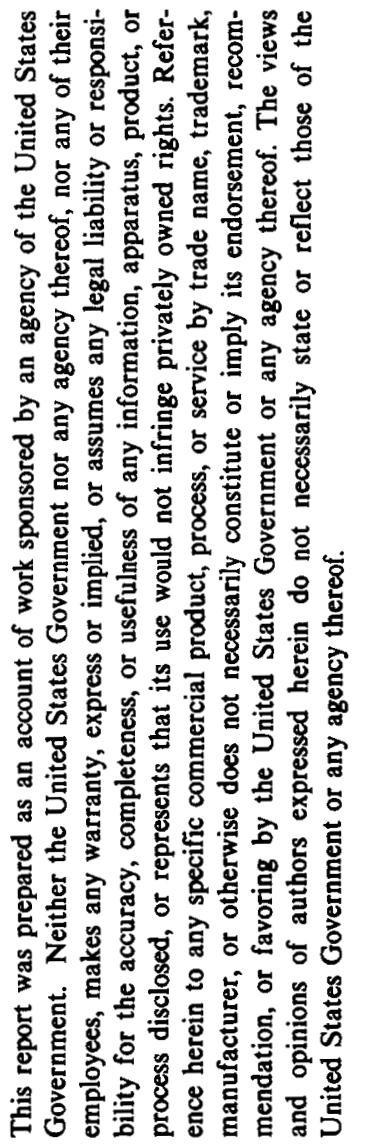

\author{
By \\ Gudmund Olsen \\ June 1984
}

Financial support was provided through the Stanford

Geothermal Program under Department of Energy Contract No. DE-AT03-80SF11459 and by. the Department of Petroleum Engineering, Stanford University. 


\section{ABSTRACT}

Depletion models for liquid-dominated geothermal reservoirs are derived and presented. The depletion models are divided into two categories: confined and unconfined. For both cases depletion models with no recharge (or influx), and depletion models including recharge, are used to match field data from the Svartsengi high temperature geothermal field in Iceland.

The influx models included with the mass and energy balances are adopted from the petroleum engineering literature. The match to production data from Svartsengi is improved when influx was included. The Schilthuis steady-state influx gives a satisfactory match. The finite aquifer method of Fetkovitch, and the unsteady state method of Hurst gave reasonable answers, but not as good. The best match is obtained using Hurst simplified solution when $\lambda=1.3 \times 10^{-4} \mathrm{~m}^{-1}$. From the match the cross-sectional area of the aquifer was calculated as $3.6 \mathrm{~km}^{2}$.

The drawdown was predicted using the Hurst simplified method, and compared with predicted drawdown from a boiling model and an empirical log-log model. A large difference between the models was obtained. The predicted drawdown using the Hurst simplified method falls between the other two.

Injection has been considered by defining the net rate as being the production rate minus the injection rate. No thermal or transient effects were taken into account. Prediction using three different net rates shows that the pressure can be maintained using the Hurst simplifled method if there is significant fluid reinjection. 
TABLE OF CONTENTS

\begin{abstract}
ACKNOWLEGEMENT

TABLE OF CONTENTS

LIST OF TABLES

LIST OF FIGURES
\end{abstract}

1. INTRODUCTION 1

2. THERMODYNAMICS 3

2.1. Liquid Only 3

2.2. Two-Phase 4

2.3. Saturation 4

3. MODELS OF LIQUID-DOMINATED GEOTHERMAL RESERVOIRS 6

3.1. Liquid Only 6

3.2. Liquid and Two-Phase Zone 8

3.2.1. Boiling Throughout the Reservoir 9

3.2.2. More Than One Lump 11

4. INFLUX MODELS 13

4.1. The Schilthuis Method 13

4.2. The Hurst Modified Method 14

4.3. General Solutions for Linear and Radial Cases 15

4.4. Hurst Simplifled Solution 15

4.4.1. Infinite Linear Aquifer 16

$\begin{array}{ll}\text { 4.4.2. Infinite Radial Aquifer } & 17\end{array}$

4.5. Superposition 18

$\begin{array}{ll}\text { 4.6. Fetkovitch } & 19\end{array}$ 
5. HISTORY MATCHING

5.1. Number of Data Points Equal to the Number of Constants to Fit 22

5.2. More Data Points Than Constants to Fit 25

5.2.1. Integrated Form $\quad 26$

5.2.2. Finite Difference Form 28

5.3. Determination of Opt imum Constants 29

6. EARLIER SVARTSENGI MODELS 32

6.1. Reservoir Description 32

6.2. Convection Model 33

6.3. Hydrological Model 34

6.4. Unit Response Function Model 35

$\begin{array}{ll}\text { 6.5. Linear Model } & 37\end{array}$

6.6. Boiling Model 38

7. DEPLETION ANALYSIS FOR SVARTSENGI 41

7.1. Liquid Models 41

7.1.1. Without Recharge $\quad 41$

$\begin{array}{ll}\text { 7.1.2. With recharge } & 43\end{array}$

7.1.2.1. Schilthuis Method 43

7.1.2.2. Hurst Modified Method $\quad 46$

7.1.2.3. Hurst Simplified Method 49

7.1.2.4. Fetkovitch Method 51

7.2. Two Zone Model 52

B. DISCUSSION

8.1. Liquid Only

8.2. Effect of Injection $\quad 55$

9. CONCLUSIONS

10. RECOMMENDATIONS 58 
REFERENCES

DEFINITIONS

APPENDIX A: Derivations of Equations

A-1

APPENDIX B: Data Files and Computer Programs

B-1

\section{LIST OF TABLES}

1 Solutions to the diffusivity equation for linear and radial geometries and different boundary conditions.

2 Example of history matching using the Schilthuis method.

\section{LIST OF FIGURES}

1 Pressure-temperature for pure water. (Whiting and Ramey, 1969)

2 Pressure-temperature for geothermal reservoirs containing pure water and a porosity of $25 \%$. (Martin, 1975)

3 Pressure-enthalpy diagram for pure water. (Whiting and Ramey, 1969)

4 Pressure vs. cumulative fluid production for geothermal reservoirs containing pure water and a porosity of $25 \%$. (Martin, 1975)

5 Temperature vs. cumulative fluid production for geothermal reservoirs containing pure water and porosity of $25 \%$. (Martin, 1975)

6 Pressure vs. steam saturation for geothermal reservoirs initially in compressed liquid state and porosity of 25\%. (Martin, 1975)

7 Steam and hot-water relative permeability curves. (Martin, 1975)

8 Lumped-parameter model. (Macias-Chapa)

9 Pressure vs. liquid saturation for a geothermal reservoir of initial temperature $240^{\circ} \mathrm{C}$, and porosity $10 \%$. (Macias-Chapa)

10 Reservoir model with three lumps. (Castanier, et al., 1980)

11 Dimensionless pressure and efflux functions for linear aquifers. (Nabor and Barham, 1964) 
12a Dimensionless water influx, constant terminal pressure, radial flow. (Van Everdingen and Hurst, 1949)

12b Dimensionless water influx, constant terminal pressure, radial flow. (Van Everdingen and Hurst, 1949)

13 Dividing continuous pressure changes into constant pressure steps for superposition.

14 Least squares fit for the example given in history matching.

15a Total mass rate produced at Svartsengi vs time.

15b Measured drawdown at Svartsengi vs. time.

16 Pressure-temperature with depth at Svartsengi.

17 The convection model for Svartsengi. (Kjaran et al. 1980)

18 Measured and calculated drawdown using the hydrological model. (Regalado, 1981)

19 Measured and calculated drawdown using the unit response function model. (Regalado, 1981)

20 The esker model (Regalado, 1981)

21 Measured and calculated drawdown using the esker model. (Regalado, 1981)

22 Measured and calculated drawdown using the boiling model with the constants reported by Thorhallsson. (1983)

23 Measured and calculated drawdown using the boiling model with the constants from our fit.

24 Drawdown vs. cumulative mass produced at Svartsengi.

25 Matching the Schilthuis model using all the data.

26 Measured and calculated drawdown for the Schilthuis model using all the data.

27 Matching the Schilthuis model without the first three points.

28 Measured and calculated drawdown for the Schilthuis model without using the first three points.

29 Fitting for $1 / a \log (b)$ and $1 / a$ in the Hurst modified method. 
30 Matching the Hurst modified method with infinite linear awuifer.

31 Measured and calculated drawdown for the Hurst modified method with infinite line ar aquifer.

32 Determination of $\lambda$ for the Hurst simplified method.

33 Measured and calculated drawdown for the Hurst simplified method.

34 Measured and calculated drawdown for a finite linear aquifer using the Fetkovitch method.

35 Measured and calculated drawdown for a finite radial aquifer using the Fetkovitch method.

36 Measured and calculated drawdown if the drainage from the two-phase zone is not instantaneous. The McNabb method.

37 Same model as in Fig.36, but using average rate.

38 Measured and calculated drawdown for the Schilthuis method based on early time data.

39 Log-log plot of measured drawdown vs. cumulative mass production.

40 Measured and calculated drawdown for the $\log -\log$ method.

41 Comparison of predicted drawdown for three models. The circles represent the log-log model, the dots represent the Hurst simplified method, and the solid line represents the boiling model for Svartsengi.

42 Prediction of the drawdown vs. cumulative production for a constant rate of $300 \mathrm{~kg} / \mathrm{s}$ using the Hurst simplified method.

43 Prediction of the drawdown vs. time for a constant rate of $300 \mathrm{~kg} / \mathrm{s}$ using the Hurst simplified method.

44 Prediction of the drawdown vs. time for three different rates using the Hurst simplified method. 


\section{INTRODUCTION}

Depletion models make it possible to predict the future variations of reservoir variables such as pressure, temperature, and chemical composition. In petroleum reservoir engineering, depletion models are used to predict the drawdown in reservoirs under a variety of production schedules. Methods developed for petroleum reservoirs involving a material balance on the reservoir have been adopted in geothermal reservoir engineering, and are the subject of this report.

In the case of geothermal fluids the heat is being mined, so it becomes important to predict the enthalpy changes with time (or production). Therefore, thermodynamics and heat transfer from the rock becomes important. Most of the heat is stored in the rock, thus a heat balance is often included in the equations. The initial state of the reservoir together with the production schedule dictates how the pressure and temperature will change upon exploitation. It therefore becomes important to determine the initial conditions of the reservoir in order to adequately describe the future behavior of the reservoir.

Depletion models can be made detailed and complex by dividing the reservoir into fine blocks and attempting to describe the variations of all reservoir parameters for each block. Parameters of interest are permeability, fluid properties, porosity, temperature, and saturation. It should be obvious that the computational methods become complicated, and that the results are limited to the input data, which are not always well known.

A simpler approach is known as lumped-parameter or zero-dimensional simulation. In this case average properties are assigned to the reservoir, and the changes of these properties are monitored and predicted. It becomes an important question then how these properties are obtained, and what the effects of material leaving and entering the system are. It is clear that these models have limitations, since the fluid as well as the rock properties are changing 
throughout the reservoir. However, it has been shown that reasonable results may be obtained from these types of models. Furthermore, important properties such as the volume of fluid in place, and whether or not there is significant recharge, may be obtained. In this study we have investigated these models to determine what models appear to be most appropriate.

Several depletion models have been reported in the literature $\mathbf{1 . 2 , 3 , 4 . 5 , 6 . 7 . 8 . 9 . 1 0}$ some of which will be considered in this study. We have attempted to make the models and computational procedures simple, and yet flexible, to be able to include a variety of liquid-dominated fields in terms of both geometry, fluid, and reservoir properties.

The influx or recharge calculations have been adopted from the petroleum literature $11,12,13,14,15$, and are therefore developed for porous media where Darcy's law is valid. It has been shown that in many cases good results may be obtained using these equations also for fractured systems. Finally the possibility of including an injection term is considered, in order to predict the pressure performance under injection.

The report consists of presentation and discussion of several depletion models and influx models. The models were evaluated using production-data from the Svartsengi field of Iceland as an example. The Svartsengi reservoir is a liquid dominated reservoir which has shown rapid drawdown, and reinjection has been considered. Derivations of the equations, presented in Appendix A, and the nomenclature are presented at the end of this report. 


\section{THERMODYNAMICS}

The thermodynamics of geothermal reservoirs have been discussed by Whiting and Ramey ${ }^{1}$ and Martin ${ }^{10}$. The initial temperature and pressure determine the state of the system. Fig. 1 is a pressure-temperature diagram for water. The solid line represents the saturation line. Gibbs' phase rule states that the number of degrees of freedom, is equal to the number of components minus the number of phases. By this, only one intensive property completely determines the thermodynamic state of the system at any point on the saturation line. For any other point on Fig.1, two intensive properties completely determine the thermodynamic state of the system.

The region of interest for this study, is in the liquid region (compressed liquid). Systems which fall in this region are liquid-dominated. This represents states such as $C$ and $B$ on Fig.1, and $C$ and $D$ on Fig.2, which in a pressuretemperature diagram for a geothermal reservoir with $25 \%$ porosity. Fig. 3 shows a pressure-enthalpy diagram for pure water. In the following, a more detailed discussion of the responses to production of reservoirs initially at states of compressed liquid, or saturated liquid-steam in equilibrium, is presented.

\subsection{Liquid Only}

The case of liquid only in the reservoir is represented by point $C$ on Fig.1, and points $\mathrm{C}$ and $\mathrm{D}$ on Fig.2. When production takes place, the pressure will decrease rapidly since the compressibility of the system is low. The path of the system is essentially isothermal and isoenthalpic (Fig.1 and Fig.3). The system will eventually reach the saturation line, and then follow a path similar to $B$ on Fig.1. 


\subsection{Two-Phase}

A two-phase reservoir corresponds to a system on the saturation-curve. The pressure-enthalpy diagram in Fig. 3 shows this state as the heavy arrow at 1000 psia. The pressure varies little when the system is boiling. This is illustrated for reservoirs with porosity of $25 \%$ in Fig.4. The pressure-cumulative fluid production diagram, shows that in reservoirs initially at compressed liquid state ( $C$ and D), the pressure drops very rapidly until the saturation-line is reached. When the reservoir follows the saturation-line, the pressure drops very slowly until the system has boiled dry, and then the pressure again drops more rapidly. The compressibility is indicated by the inverse of the slope of the lines on Fig.4. Fig.5 shows the temperature vs. cumulative fluid production for geothermal reservoirs containing pure water and porosity of $25 \%$. It shows that the temperature drops when there are two-phases in the system. Since the fluid temperature wants to decrease, heat may in this case be transferred from the surrounding rock where most of the heat is stored ${ }^{2}$.

\subsection{Saturation}

Lumped-parameter models can be developed for the two above cases (liquid only and two-phase reservoir) if the variation of saturation with pressure is known. Martin ${ }^{16}$ developed such relations. The results for a $25 \%$ porosity system are given in Fig. 6 that shows the variation of saturation with pressure. The equations presented include Darcy's law for each phase, steam and liquid water, and thus also relative permeability data. The relative permeability curves used by Martin $^{16}$ are given in Fig.7. Darcy's law was then coupled with the mass and heat continuity equations. Eq. 1 shows the final expression for saturation.

$$
\frac{d S_{w}}{d p}=\frac{\Lambda_{h}\left(\frac{\partial m_{f}}{\partial p}\right)-\Lambda_{f}\left(\frac{\partial H}{\partial p}\right)}{\Lambda_{f}\left(\frac{\partial H}{\partial S_{w}}\right)-\Lambda_{h}\left(\frac{\partial m_{f}}{\partial S_{w}}\right)}
$$


where:

$$
\begin{aligned}
& \Lambda_{h}=\frac{\left(\rho_{w} k\right)}{\mu_{w}}+\frac{\left(\rho_{s} h_{s} k\right)\left(k_{r s}\right)}{\mu_{s}}+k_{h} \psi^{\prime} \\
& \Lambda_{f}=\frac{\left(\rho_{w} k\right)\left(k_{r w}\right)}{\mu_{w}}+\frac{\left(\rho_{s} k\right)\left(k_{r s}\right)}{\mu_{s}} \\
& m_{f}=\varphi\left(\rho_{w} S_{w}+\rho_{s} S_{s}\right) \\
& H=\varphi\left[\rho_{w} h_{w} S_{w}+\rho_{s} h_{s} S_{s}+\left(\frac{1-\varphi}{\varphi}\right) \rho_{s} C_{\sigma} \psi\right] \\
& \psi(p)=\text { temperature on boiling curve. } \\
& \psi^{\prime}=\frac{d \psi(p)}{d p}
\end{aligned}
$$

Eq. 1 was numerically integrated to get the pressure vs. steam saturation curves given in Fig. 6 for reservoirs initially at compressed liquid state.

Macias-Chapa ${ }^{17}$ developed a lumped-parameter model where the system is described by Fig.8. Production may be steam only, or liquid/steam, or only liquid. A computer program was written to calculate the adiabatic or the apparent compressibilities including effects of heat transfer from the rock. In all cases the compressibility for a two-phase water system is much greater than that of only steam or liquid water.

The model includes impurities in form of non-condensable gasses and dissolved solids. The system is on the saturation-line, and after the pressure has been decreased by a specified amount, the saturation may be determined. An output of saturation vs. pressure for a $10 \%$ porosity and initial temperature of $240^{\circ} \mathrm{C}$, is given in Fig.9. The solid line represents a cubic fit trough the output values from the program. This relationship may be used in modeling the saturation variation with pressure. 


\section{MODELS OF LUUID-DOMINATED GEOTHERMAL RESERVOIRS}

\subsection{Liquid Only}

For reservoirs with liquid only, the production path, as described earlier, is for all practical purposes isothermal and isoenthalpic. The heat balance is thus omitted. The discussion may be divided into two cases: confined and unconfined systems. In a confined system, the production is due to expansion of the compressed fluid. The unconfined system is referred to as open. When this system is produced, the water level decreases in the same manner as emptying a tank. The reservoir in both cases is treated as one lump with average properties.

\subsubsection{Unconfined System}

In the simplest form the equation for the drawdown is given by:

$$
\Delta h=\frac{W_{p}}{A \varphi \rho_{w}}
$$

(See Appendix A for derivations of the equations).

If Eq. 2 is valid, the drawdown plotted vs. cumulative mass produced should give a straight line. However, if the points fall on a straight line, this does not guarantee that there is no recharge, or that the system is not confined. If the reservoir is produced at constant rate, the line could be straight even with strong recharge. The slope of the line would be different, but if there is a steady state influx, and constant discharge rate, the drawdown would be similar to that of no influx. Furthermore, if the comressibility is constant, the graph would be similar. On the other hand, even if the graph is not a straight line, there could be an unconfined system without recharge since $A \varphi \rho_{w}$ may change with production.

If there is recharge to the system, the rate of mass removed from the system becomes important. This question will be discussed in detail later, but one 
"particularly simple equation for the recharge will be presented here. In this equation which is known as the Schilthuis ${ }^{19}$ steady state equation, the influx rate is proportional to the drawdown.

$$
\frac{A \varphi}{g} \frac{d p}{d t}=K\left(p_{i}-p\right)-w
$$

In this model there are no transients in the reservoir, and the pressure distribution in the reservoir is hydrostatic.

It is possible that $\varphi$ or $A$ are functions of depth, in which Eq. 2 and Eq.3 are no longer valid, and the equations must be modified. By looking at resistivity measurements, it sometimes looks like the reservoir is pyramid, or cone shaped. In that case the area is a linear function of the height, and the solution would be:

$$
h_{i}^{2}-h^{2}=\frac{W_{p}}{a \varphi \rho_{w}}
$$

In this case there should see a straight line if cumulative production is plotted vs. $\left(h_{i}^{2}-h^{2}\right)$. Notice that $h_{i}^{2}-h^{2} \neq(\Delta h)^{2}$, so it becomes important in this case to identify $h_{i}$. Eq.4 could also be coupled with influx models. The procedure is analogous to what will be presented later in this study.

\subsubsection{Confined Systems}

This system is a in compressed liquid state, and the production is due to expansion of the liquid when the pressure drops. The equations for this system have been presented by Whiting and Ramey ${ }^{1}$ and applied to the Wairakei geothermal field:

$$
V \varphi\left(\frac{d \rho_{w}}{d p}\right)_{T} \frac{d p}{d t}=\frac{d W_{e}}{d t}-w
$$

Comparing Eq. 3 and Eq.5 shows that if $\left(d \rho_{w} / d p\right)_{T}$ is constant, the two equations are of the same form. The constants in front of $d p / d t$ are different in the two equations. The size of this constant helps identify which model is most rea- 
sonable for a given reservoir. Eq. 5 may be rewritten by introducing the compressibility of water:

$$
V \varphi \rho_{w} c_{w} \frac{d p}{d t}=\frac{d W_{B}}{d t}-w
$$

A variety of influx equations with various geometries and boundary conditions are possible. The influx models are discussed in a separate chapter.

When integrating Eq. 6 to get the drawdown, it is assumed that $c_{w}$ and $\rho_{w}$ are constant. This is a good approximation for a relatively small pressure change, and will be a good approximation in the early development when the drawdown is not too severe. However, over a long prediction this may not be a good assumption. This is especially true when boiling starts occurring in the reservoir. It may therefore be necessary to update $p_{w}$ and $c_{w}$ as time goes on by discretizing Eq.6, and calculate increments of production and drawdown.

\subsection{Liquid and Two-Phase Zone}

When the pressure in the reservoir falls below the boiling point, a two-phase zone will develop. The depth of geothermal reservoirs is sometimes several kilometers, so the pressure will vary with depth. This makes it difficult to assign average properties to the entire reservoir when there is boiling in some parts of the reservoir. In some reservoirs the temperature profile follows the boiling point with depth curve. In that case boiling will start throughout the reservoir, and the models presented here will apply. But many reservoirs are essentially isothermal with depth due to convection. It is clear that in this case, the fluids closest to the surface will start to boil first. Low in the reservoir (except for local low-pressure regions around the wells), boiling will normally not occur due to the higher pressure.

Martin ${ }^{18}$ discussed how rapid gravity segregation of liberated steam causes a zone of two-phases to develop at the top of the reservoir with higher liquid sa- 
turation deeper in the reservoir. The question of being able to define an average liquid saturation for the whole reservoir and the total compressibility of the system becomes very important. For a detailed analysis of the system, it may be necessary to divide the reservoir into several lumps.

\subsubsection{Boiling Throughout the Reservoir}

When saturation pressure and temperature are reached, the system follows the saturation curve (Fig.1). Since energy is transferred in the process of evaporization, and heat is transferred between the rock and the fluid, a heat balance must be included. The heat and energy balance was written by Whiting and Ramey as:

$$
\begin{aligned}
& W_{p}\left(h_{p}-E_{c}\right)+W_{l}\left(h_{l}-E_{c}\right)+Q= \\
& W_{i}\left(E_{i}-E_{c}+\left(\frac{1-\varphi}{\varphi}\right)\left[x_{i} \nu_{s i}+\left(1-x_{i}\right) \nu_{w i}\right] p_{\sigma} C_{v \sigma}\left(T_{i}-T_{c}\right)\right)+ \\
& \left(h_{e}-E_{c}\right) \frac{B}{v_{w e}} \sum W_{D}\left(t_{D}\right) \Delta p_{n}
\end{aligned}
$$

This equation was developed for predicting the response of the Wairakei reservoir. When trying to match the data, it was concluded that the system was initially in a compressed liquid state, for which Eq. 7 reduces to:

$$
\left(W_{p}+W_{l}\right) \nu_{w}=W_{i}\left(\nu_{w}-v_{w i}\right)+B \sum W_{D}\left(t_{D}\right) \Delta p
$$

Eq. 8 is a volumetric balance where the last term is the influx term, and is essentially the basis for Eq.5. If we define the total density of the system and assume that the mass loss can be neglected. the mass balance becomes:

$$
V \varphi \rho_{t}=V \varphi \rho_{t i}+W_{e}-W_{p}
$$

In this equation the total density is a function of the density of each phase and the saturation: If we are able to get a relationship between the saturation and the pressure, Eq.9 could be used to calculate the drawdown. The procedure is discussed in the chapter on history matching. 
In order to use either Eq.7 or Eq.9, the initial state of the system must be known. In the chapter on history matching, methods to determine optimum initial parameters are described. Notice that the heat balance is not included in Eq.9. The heat balance is included in the relationship to determine the saturation, and is therefore present in the determination of the total density. The temperature and enthalpy are fixed, once the pressure has been determined, since we have saturated conditions.

A different equation was presented by $\mathrm{McNabb}^{4.5,6}$. The drainage from the two-phase zone was assumed not to be instantaneous, and the variation of saturation above the declining boiling level had to be described. If the relative permeability to liquid water is assumed linear with saturation, and that rapid draining fractures are surrounding less permeable porous blocks, the equation for the pressure is:

$$
\frac{d p}{d t}=a\left(p_{i}-p\right)+b w+c \frac{d w}{d t}
$$

Eq.10 was also applied to the Wairakei field, giving reasonable answers 4 . The coefficients in Eq.10 can be expressed in terms of $A \varphi, k$, and $S_{w_{0}}{ }^{6}$ :

$$
\begin{aligned}
& a=\frac{K \frac{g}{A \varphi} \tau_{0}^{-1}}{K \frac{g}{A \varphi}+\left(1-S_{w_{0}}\right) \tau_{0}^{-1}} \\
& b=\frac{\frac{g}{A \varphi} \tau_{0}^{-1}}{K \frac{g}{A \varphi}+\left(1-S_{w_{0}}\right) \tau_{0}^{-1}} \\
& c=\frac{\frac{g}{A \varphi}}{\frac{g}{A \varphi}+\left(1-S_{w_{0}}\right) \tau_{0}^{-1}}
\end{aligned}
$$

In the above equations $K$ is the Schilthuis influx constant used in Eq.3, and $\tau_{0}$ is a characteristic time of the fractured permeable medium. 


\subsubsection{More Than One Lump}

Castanier et al. ${ }^{10}$ divided the reservoir into three lumps as shown in Fig.10. The central zone from which the production occurs, is represented by a lumped parameter model to predict the production of mass and energy. This zone is treated as a homogeneous tank, and the production of mass and enthalpy was calculated using the following mass and energy balance:

$$
W_{c} E_{c}+V(1-\varphi) \rho_{\sigma} C_{v \sigma}\left(T_{c}-T_{i}\right)-W_{i} E_{i}+Q=W_{\varepsilon} h_{i}-W_{p} h_{p}-W_{l} h_{l}
$$

Eq.11 is the same as Eq.7 as presented by Whiting and Ramey ${ }^{1}$. However, there is an intermediate zone in which neither production nor injection occurs. Nevertheless, there is heat and mass transfer in this zone during depletion. Mass transfer occurs from the outside to the inner production zone. The temperature breakthrough time of the fluid leaving this zone (entering the inner zone), is computed using the fluid breakthrough time multiplied by ${ }^{10}$.

$$
\frac{\varphi \rho_{w} C_{w}+(1-\varphi) \rho_{\sigma} C_{0}}{\varphi \rho_{w} C_{w}}
$$

Finally the outer zone has fluid flow only. There is no heat flow in this zone, only natural recharge or fluid injection.

For each depletion step, the production of mass and energy is calculated. In order to calculate the time taken by this step of depletion, constraints on production such as constant enthalpy, flow rate, or available energy is taken into account. The pressure distribution in the central zone is then calculated using a superposition of pressure distributions of off-centered wells. Finally the water and heat influx from the intermediate zone are calculated. The entire process is repeated until abandonment conditions are reached.

This model was compared to a reservoir simulation study by Morris and Cambell ${ }^{18}$ of the East Mesa reservoir in the Imperial Valley, and good agreements were obtained. It was concluded that although being able to handle fluid 
flow and heat transfer more realistically compared to a one lump model, this method is simpler and less expensive than a three-dimensional finite difference simulation model. It should also be noted that in the limit of the intermediate zone having zero width, this model reduces to a lumped parameter model similar to the Whiting and Ramey ${ }^{1}$ model. 


\section{INFLUX MODEIS}

If the drawdown history of a geothermal reservoir can not be explained by simple mass removal, there may be water influx or recharge into the reservoir. Recharge will maintain pressure in the reservoir, by replacing the produced fluids by usually colder fluids. A term of influx mass has to be added to the mass balance equations. Assuming no mass loss, the mass balance becomes Eq.A-2:

$$
W_{c}=W_{i}-W_{p}+W_{e}
$$

\subsection{The Schilthuis Method}

In the Schilthuis ${ }^{10}$ steady-state model, the influx rate is equal to a constant, times the pressure change. If the outer boundary pressure of the aquifer is assumed to always be at initial pressure, and that there is steady state flow, the influx rate may be given by Darcy's law as:

$$
w_{\mathrm{a}}=\rho A u=\frac{k A \rho}{\mu} \frac{\Delta p}{\Delta L}
$$

in the linear case. For different geometries, Eq.14 has a different constant, but in all cases:

$$
w_{e}=K \Delta p
$$

where $\Delta p=p_{i}-p . K$ is known as the influx constant. To get the cumulative influx, the rate is given by:

$$
\frac{d W_{e}}{d t}=w_{e}
$$

Integrating Eq.15 (using Eq.14) gives:

$$
W_{e}=\int_{0}^{t} w_{e} d t=K \int_{0}^{t}\left(p_{i}-p\right) d t
$$

If pressure is known as a function of time, Eq.17 can be integrated. If not, numerical integration yields:

$$
W_{o n}=K \sum_{j=1}^{n}\left(p_{i}-p_{j}\right) \Delta t_{j}
$$


This is the simplest form of an influx equation and expresses the cumulative mass recharge in a steady state system. It has been presented in the geothermal literature with models applied to the Wairakei reservoir. ${ }^{4.5 .6}$ and the Bangore reservoir. ${ }^{32}$

\subsection{The Hurst Modified Method}

Sometimes the transient effects are important in aquifer behavior and the aquifer may act as infinite in extent. For this case a well known solution to the diffusivity equation is the line source solution. For large values of $t_{D}$, the log approximation can be used for the line source solution $\left(t_{D}>10\right)$ :

$$
p_{D}=\frac{1}{2}\left[\ln \left(t_{D}\right)+0.80907\right]
$$

By the definition of $t_{D}$, it becomes apparent that Eq.18 is valid for large times and small radii. By introducing the definition of $p_{D}$ for radial geometry, Eq.18 becomes:

$$
\frac{1}{2}\left[\ln \left(t_{D}\right)+0.80907\right]=\frac{2 \pi k h \Delta p}{q_{B} \mu}
$$

Multiplying by $q_{\mathrm{e}}$ and rewriting gives:

$$
q_{0}=\frac{2 \pi k h \Delta p}{\frac{1}{2} \mu\left[\ln \left(t_{D}\right)+0.80907\right]}
$$

Since $q_{e}=\frac{w_{e}}{\rho}$, if constant $\mu$ is assumed, te mass rate can be expressed as:

$$
w_{\mathrm{a}}=\frac{a \Delta p}{\log (b t)}
$$

and the influx is given by:

$$
W_{c}=\int_{0}^{t} w_{s} d t=\int_{0}^{t} \frac{a \Delta p}{\log (b t)} d t
$$

where $\mathrm{a}$ and $\mathrm{b}$ are constants. Eq.22 is usually numerically integrated:

$$
W_{a n}=\sum_{j=1}^{n} \frac{a \Delta p_{j} \Delta t_{j}}{\log \left(b \bar{t}_{j}\right)}
$$


.where $\bar{t}_{j}=\left(t_{j}+t_{j-1}\right) / 2$, the average time for the j'th step of depletion.

\subsection{General Solutions for Linear and Radial Cases}

The type of influx given in Eq.23, is generally known as the Hurst modified solution. Van Everdingen and Hurst ${ }^{20}$ presented solutions to the influx equations similar to Eq.18 for different boundary conditions in the radial case. Miller ${ }^{11}$ presented solutions for the linear case. The work by Miller ${ }^{11}$ was later extended by Nabor and Barham ${ }^{12}$ to include solutions for a constant pressure outer boundary. By changing the definition of $t_{D}$, they came up with three working equations for all boundary conditions.

The solutions to these problems are summarized in Table 1. Tables of numerical values for these solutions have been presented in the literature. ${ }^{15.20}$ The solutions can also be presented in graphical form: Fig.11 is the linear case, and Fig.12a and Fig.12b the radial cases.

Van Everdingen and Hurst ${ }^{20}$ presented how the cumulative water influx is calculated in terms of $Q_{D}$. The equations assume that the inside pressure is constant. For a constant pressure drop, the cumulative water influx is given as a function of time by:

$$
W_{\mathrm{a}}=B Q_{D}\left(t_{D}\right) \Delta p
$$

For varying pressure, the method of superposition, is used as discussed in a later section.

\subsection{Hurst Simplified Solution}

The equations presented in Table 1 were solved using the Laplace transformation. ${ }^{20}$ By coupling the influx equations with the material balance equation on the reservoir, a particularly interesting solution arises. Hurst ${ }^{19}$ presented this solution for a petroleum reservoir. A geothermal reservoir with only liquid, is 
analogous to a petroleum reservoir above the bubble point pressure. In this case the compressibilities in the reservoir and the aquifer may be treated as constants, and the equations may be solved using the Laplace transformation. Two solutions are presented here: the infinite linear and infinite radial cases. However, the method can be used with any geometry if $\bar{Q}_{D}$ is known in Laplace space. Sometimes an analytical transformation is not possible. In that case, numerical methods can be used.

\subsubsection{Infinite Linear Aquifer}

By defining $\lambda=\frac{c_{\text {aq }} \rho_{\text {aq }}}{l c_{\text {res }} \rho_{\text {res }}}$. Hurst ${ }^{13}$ was able to present two limiting solutions.

1. $\lambda$ very large

In this case the aquifer becomes the important factor in the response: the reservoir is ignored. The compressibility of the reservoir is negligible, so this corresponds to an unconfined system with infinite linear influx. Note that this case will also occur when $l \rightarrow 0$. The solution then reduces to the aquifer solution only, and the drawdown is given by:

$$
\Delta p=\frac{\mu}{k A} \sum_{j=0}^{n} \Delta w_{j} F_{\frac{1}{2}}\left(t_{D}-t_{D j}\right)
$$

Eq.24 is the superposition sum for varying rate, and $F_{1 / 2}$ is the solution for an infinite linear system given by Nabor and Barham. ${ }^{12}$

\section{2. $\lambda$ very small}

In this case the reservoir becomes the dominant factor, and if the compressibility of the reservoir is large but constant, this situation may be occurring. The aquifer is now completely ignored, and the equation for the drawdown simply reduces to the tank decompression in Eq.6 without recharge. The system is now confined:

$$
\Delta p=\frac{1}{V \varphi c_{\text {res }} \rho_{\text {res }}} w_{p}
$$




\section{3. $\lambda$ intermediate}

The effects of both the reservoir and the aquifer become important, and the total expression must be used. This situation lies between the limiting cases of confined and unconfined. For the infinite linear case, an analytical solution exists. In cases where such inversions are not possible, there are numerical methods that transform a solution from Laplace space to real space. One such method is the Stehfest ${ }^{21}$ algorithm. The equation for the drawdown becomes:

$$
\Delta p=\frac{\mu_{\text {oq }} c_{\text {oq }}}{k V \rho_{\text {res }} c_{\text {res }}} \sum_{j=0}^{n} \Delta w_{j} M\left[\lambda^{2}\left(t_{D}-t_{D_{j}}\right)\right]
$$

where

$$
M\left(\lambda^{2} t_{D}\right)=\frac{1}{\lambda^{2}}\left(e^{\lambda^{2} t_{D}} \operatorname{erfc}\left(\lambda t_{D}^{\frac{1}{2}}\right)-1+\frac{2 \lambda t_{D}^{\frac{1}{2}}}{\pi^{\frac{1}{2}}}\right)
$$

\subsubsection{Infinite Radial Aquifer}

The solution is analogous to the linear case. In this case define $\lambda_{r}=\frac{2 c_{e q} \rho_{a q}}{c_{\text {res }} \rho_{\text {res }}}$

1. $\lambda_{r}$ very large

Again the system acts as if the reservoir is not there. The drawdown is given by the solution for a infinite radial system

$$
\Delta p=\frac{\mu}{2 \pi k h p} \sum_{j=0}^{n} \Delta w_{\theta} p_{D}\left(t_{D}-t_{D j}\right)
$$

For $t_{D} \geq 10$ we can use Eq.18. Complete solutions are given by Van Everdingen and Hurst ${ }^{20}$ and Chatas ${ }^{15}$.

2. $\lambda_{r}$ very small

Again the aquifer is ignored, and tank decompression is the solution. Therefore the equations reduce to Eq.25 in this case also. 
3. $\lambda_{r}$ intermediate

Now the entire expression must be used. In this case there is no analytical solution available. Solutions were presented in graphical form by Hurst ${ }^{19}$. The drawdown is given by

$$
\Delta p=\frac{\mu_{a q}}{2 \pi k h \rho_{\alpha q}} \sum_{j=0}^{n} \Delta w_{\varepsilon j}\left[\sigma N\left(\sigma, t_{D}-t_{D j}\right)\right]
$$

where

$$
\sigma N\left(\sigma, t_{D}\right)=L^{-1}\left(\frac{K_{0}(\sqrt{s})}{s^{\frac{s}{2}}\left[\sigma K_{2}(\sqrt{s})+\sqrt{s} K_{0}(\sqrt{s})\right]}\right)
$$

\subsection{Superposition}

Since the equations presented in the petroleum literature are limited to the boundary conditions of either constant rate, or constant pressure at the inner boundary, it becomes important to be able to modify the equations take into account varying pressure or rate. This is done by dividing the changes into discrete steps, and adding the effects of each constant pressure step. The method for doing this is known as the principle of superposition, and can be applied in both space and time (see Fig.13).

The idea is that the effects on a point in space is the algebraic sum of the effects from each contributing change. For example, to determine the effects of several wells in a reservoir on the pressure at a certain location in the reservoir. simply calculate the effect from each well assuming there are no other wells in the reservoir, and then add the pressure changes from each well to get the total effect. Similarly the effects of several changes at different points in time on a point later in time, are additive. The principle of superposition is discussed in more detail in petroleum engineering textbooks and was discussed by Van Everdingen and Hurst ${ }^{13}$ 
- Using the Van Everdingen and Hurst equation (Eq.23), the influx is given by the superposition theorem as:

$$
W_{0}=B \sum_{j=0}^{n} Q_{D}\left(t_{D}-t_{D j}\right) \Delta p_{j}
$$

In this equation $t_{D}$ represents current time. The initial pressure drop is effective from the beginning until present time, and the additional pressure drops are in effect for successively shorter times. This is illustrated on Fig.13. It should also be noted that in the limit of infinite steps, Eq.29 may be written in integral form. This is known as the convolution integral, and can be used directly if the variation of pressure as a function of time is known. Since the limit of the integration is from initial to present, and that the initial conditions are generally known for reservoirs, this integral is suited for solutions by the Laplace transformation. The integral is given by:

$$
W_{a}=B \int_{0}^{t_{D}} \frac{d \Delta p}{d t_{D}^{\dot{B}}} Q_{D}\left(t_{D}-t_{\dot{D}}\right) d t_{D}
$$

\subsection{Fetkovitch}

It becomes complicated to always have to use the superposition theorem, especially if computers are not available, and if the reservoir is closed since there are no simple analytical solutions to the problem. A different method was developed by Fetkovitch, ${ }^{22}$ which is especially useful with closed reservoirs. In this method the average pressure of the reservoir is assumed to be the inside pressure of the aquifer. The average pressures of the reservoir and the aquifer are calculated using material balance. For each time-step (the size of the timesteps need not be equal), the average pressure in the reservoir is held constant, while the average pressure in the aquifer is allowed to decrease.

The method is based on defining a resistance function between the aquifer and the reservoir. Since a finite reservoir which is closed is assumed, pseudo- 
steady state is assumed to be reached immediately. This is not true, but the time to reach pseudo-steady state is usually much smaller than the time for the prediction. The equations for pseudo-steady state flow for radial geometry are:

$$
w_{e}=\frac{2 \pi k h\left(p_{a v}-p_{w f}\right)}{\mu \frac{r_{e}^{4}}{\left(r_{e}^{2}-r_{w}^{2}\right)^{2}}\left(\ln \frac{r_{e}}{r_{w}}-\frac{3}{4}+\left(\frac{r_{w}}{r_{e}}\right)^{2}-\frac{1}{4}\left(\frac{r_{w}}{r_{a}}\right)^{4}\right)}
$$

which if $\frac{r_{\varepsilon}}{r_{w}}>10$ reduces to

$$
w_{c}=\frac{2 \pi k h\left(p_{a v}-p_{w f}\right)}{\mu\left[\ln \frac{r_{a}}{r_{w}}-\frac{3}{4}\right]}
$$

for linear geometry:

$$
w_{e}=\frac{k b h\left(p_{a v}-p_{w}\right)}{\mu \frac{L}{3}}
$$

The method is based on a constant productivity index. For a closed system, the rate may be expressed as (see Appendix B of Fetkovitch ${ }^{22}$ )

$$
w_{c}=J_{w}\left(p_{i}-p_{w f}\right) \exp \left(-\frac{w_{e i, \max }}{W_{e i}} t\right)
$$

Eq.33 is the most important equation in this method. The procedure for doing the actual calculations are as follows:

1. Calculate $w_{\text {si.max }}$ (the maximum influx rate using one of the equations for pseudo-steady state flow with $p_{w f}=0$ ).

2. Calculate $A_{n}$ for the time step.

$$
A_{n} \equiv \frac{W_{s i}}{p_{i}}\left[1-\exp \left(-\frac{w_{B i, m a x}}{W_{a i}} \Delta t_{n}\right)\right]
$$

where

$$
W_{\mathrm{ei}}=V_{\mathrm{aq}} \rho \varphi c_{t w} p_{i}
$$

3. Calculate the average pressure in the reservoir after the end of the time step. This is $p_{w f(n)}$ in the aquifer. For a constant compressibility decompres- 
sion

$$
P_{w f(n)}=\frac{-W_{p_{n}}+W_{e_{n-1}}+A_{n}\left(p_{w(n-1)}-\frac{p_{w \rho(n-1)}}{2}\right)+p_{i}\left(V \varphi \rho_{r e s} c_{r e s}\right)}{V_{\varphi \rho_{\text {res }} c_{\text {res }}+\frac{A_{n}}{2}}}
$$

If the reservoir is unconfined, the material balance in the reservoir is given by Eq.3, and then Eq. 35 will have $V \varphi \rho_{\text {res }} c_{\text {res }}$ replaced by $\frac{A \varphi}{g}$.

4. Calculate the incremental influx for this time step.

$$
\Delta W_{a_{n}}=\left(p_{a v(n-1)}-\frac{p_{w f(n-1)}}{2}-\frac{p_{w f(n)}}{2}\right) A_{n}
$$

5. Calculate the average pressure in the aquifer at the end of the time step

$$
p_{a v(n)}=p_{i}-\frac{p_{i}}{W_{a i}}\left(W_{e_{n-1}}+\Delta W_{B_{n}}\right)
$$

6. Finally we can calculate the cumulative influx at the end of the time step

$$
W_{a_{n}}=W_{e_{n-1}}+\Delta W_{a_{n}}
$$

If the pressure can be represented by the hydrostatic column of water in the observation well, the drawdown over the time step is given by

$$
\Delta h_{n}=\Delta h_{n-1}+\frac{p_{w f(n-1)}-p_{w f(n)}}{\rho_{\text {res }} g}
$$

The procedure is then repeated from step 2. This method is especially suited for solving on a programmable calculator, or a computer. It is possible to update the compressibility and the density as time goes on. Using data on drawdown and rate, one can determine the constants that will give the best results. Note also that for this method, the initial pressure in the system must be identified. 


\section{HISTORY MATCHING}

After a geothermal reservoir has been produced for a period of time, a depletion model can be matched to the production data. The production history is used to obtain optimum parameters of a particular model. As more data becomes available, more and more becomes known about the reservoir. With time there are data available which may be used to improve the understanding of the reservoir. This becomes important in future modeling of other similar reservoirs. In all cases data must be collected as the reservoir is produced. The model is limited to the data used, so all the pressure responses must be included.

In matching production data it is possible to get as many parameters as there are data points. If there are three data points it is possible to fit three constants. A more usual situation is that there are more data points than constants to fit. In that case, the method of least squares fitting may be used. It is also possible to use graphical techniques. These concepts are best illustrated by examples.

\subsection{Number of Data Points Equal to the Number of Constants to Fit}

A drawdown of 10 meters is shown after producing $4 \times 10^{9} \mathrm{~kg}$ of water. The reservoir is filled with liquid of density $870 \mathrm{~kg} / \mathrm{m}^{3}$, and from resistivity measurements the volume was estimated to be $3.5 \times 10^{9} \mathrm{~m}^{3}$. The porosity from core samples is thought to be $25 \%$. No recharge is thought to be significant.

There is only one data point available, so only one constant can be determined. For liquid only the possibilities of a confined or an unconfined system can be tried. The compressibility of liquid water at these conditions is about 1. $2 \times 10^{-9} \mathrm{~Pa}^{-1}$. If the system is confined, Eq.6 is used in an integrated form without recharge: 


$$
V \varphi \rho_{w} c_{w}\left(p-p_{i}\right)=-W_{p}
$$

which becomes:

$$
V \varphi \rho_{w}^{2} c_{w} g \Delta h=W_{p}
$$

Solving for the volume gives:

$$
V=\frac{W_{p}}{\varphi \rho_{w}^{2} c_{w} g \Delta h}
$$

Substituting the values gives:

$$
V=\frac{4 \times 10^{9} \mathrm{~kg}}{(0.25)(870)^{2} \frac{\mathrm{kg}^{2}}{\mathrm{~m}^{8}}\left(1.2 \times 10^{-9}\right) \mathrm{Pa}^{-1}(9.81) \frac{\mathrm{m}}{\mathrm{s}^{2}}(10) \mathrm{m}}=1.8 \times 10^{11} \mathrm{~m}^{3}
$$

which is much higher than the believed $3.5 \times 10^{9} \mathrm{~m}^{3}$. If the system is unconfined, Eq. 2 is used and solving for A gives:

$$
A=\frac{W_{p}}{\varphi p_{w} \Delta h}
$$

Using the values given:

$$
A=\frac{4 \times 10^{9} \mathrm{~kg}}{(0.25)(870) \frac{\mathrm{kg}}{\mathrm{m}^{\mathrm{g}}}(10) \mathrm{m}}=1.8 \times 10^{6} \mathrm{~m}^{2}
$$

If the volume is $3.5 \times 19^{9} \mathrm{~m}^{3}$, this would give a vertical height of:

$$
h=\frac{V}{A}=\frac{3.5 \times 10^{9} \mathrm{~m}^{3}}{1.8 \times 10^{8} \mathrm{~m}^{2}}=1900 \mathrm{~m}
$$

This number is more reasonable, indicating that an unconfined reservoir is more likely.

If an unconfined model appears reasonable, what will the drawdown be after 30 years of production if the rate is constant at $100 \mathrm{~kg} / \mathrm{s}$ ?

The total mass produced after 30 years is:

$$
W_{p}=w t=(100) \frac{\mathrm{kg}}{\mathrm{s}}(30) \text { years }\left(31.536 \times 10^{6}\right) \frac{\mathrm{sec}}{\text { years }}=9.46 \times 10^{10} \mathrm{~kg}
$$

Added to the produced fluid when the drawdown was 10 meters, the total mass produced in 30 years will be: 


$$
W_{p t}=9.46 \times 10^{10} \mathrm{~kg}+4 \times 10^{9} \mathrm{~kg}=9.86 \times 10^{10} \mathrm{~kg}
$$

The drawdown is obtained from Eq.2:

$$
\Delta h=\frac{W_{p t}}{A \varphi \rho_{w}}=\frac{9.86 \times 10^{10} \mathrm{~kg}}{\left(1.8 \times 10^{6}\right) \mathrm{m}^{2}(0.25)(870) \frac{\mathrm{kg}}{\mathrm{m}^{3}}}=252 \mathrm{~m}
$$

Now assume that when $10 \times 10^{10} \mathrm{~kg}$ was produced, the drawdown was measured as $150 \mathrm{~m}$. Therefore, the model is predicting too large a drawdown. This may show that there is some pressure maintenance in the system.

Assume that a more careful study showed that there is influx from a permeable zone along a fault. Two constants can now be obtained since there are two data points. Notice that the temperature and enthalpy are assumed constant so the heat balance can be ignored. If a Schilthuis ${ }^{19}$ type equation is assumed, Eq.3 can be used. The integrated form of Eq. 3 becomes:

$$
\frac{A \varphi}{g}\left(p-p_{i}\right)=K\left(p_{i}-p\right) t-w t
$$

Since the rate has been assumed constant, $W_{p}=w t$. Writing this equation for the two data points and since $p=p g h$ :

$$
\begin{aligned}
& -A \varphi \rho_{w} \Delta h_{1}=K \Delta h_{1} t_{1}-W_{p 1} \\
& -A \varphi \rho_{w} \Delta h_{2}=K \Delta h_{2} t_{2}-W_{p 2}
\end{aligned}
$$

Solving the first equation for $A \varphi \rho_{w}$ gives:

$$
-A \varphi p_{w}=\frac{K \Delta h_{1} t_{1}-W_{p 1}}{\Delta h_{1}}
$$

Substituting in the second equation gives:

$$
\frac{K \Delta h_{1} t_{1}-W_{p 1}}{\Delta h_{2}} \Delta h_{2}=K \Delta h_{2} t_{2}-W_{p 2}
$$

Solving for the influx constant gives:

$$
K=\frac{W_{p 1}-W_{p 2} \frac{\Delta h_{1}}{\Delta h_{2}}}{\Delta h_{1} t_{1}-\Delta h_{1} t_{2}}
$$

where $t_{1}=4 \times 10^{7} \mathrm{~s}$, and $t_{2}=9.46 \times 10^{8} \mathrm{~s}$. Substituting the values given, an expres- 
sion for $\mathrm{K}$ is obtained:

$$
K=\frac{\left(4 \times 10^{9}\right) \mathrm{kg}-\left(9.86 \times 10^{10}\right) \mathrm{kg} \frac{10}{150}}{(10) \mathrm{m}\left(4 \times 10^{7}\right) \mathrm{s}-(10) \mathrm{m}\left(9.46 \times 10^{8}\right) \mathrm{s}}=0.28 \frac{\mathrm{kg}}{\mathrm{m} \cdot \mathrm{s}}
$$

using this and substituting in the first equation:

$$
A \varphi \rho_{w}=\frac{\left(4 \times 10^{9}\right) \mathrm{kg}-(0.28) \frac{\mathrm{kg}}{\mathrm{m} \cdot \mathrm{s}}(10) \mathrm{m}\left(4 \times 10^{7}\right) \mathrm{s}}{10 \mathrm{~m}}=3.9 \times 10^{8} \frac{\mathrm{kg}}{\mathrm{m}}
$$

With $\varphi=0.25$ and $\rho_{w}=870 \mathrm{~kg} / \mathrm{m}^{3}$, the area becomes:

$$
A=\frac{3.9 \times 10^{8} \frac{\mathrm{kg}}{\mathrm{m}}}{(0.25)(870) \frac{\mathrm{kg}}{\mathrm{m}^{3}}}=1.8 \times 10^{6} \mathrm{~m}^{2}
$$

This is the same as found in the first example. Now the drawdown after 50 years can be predicted. After 30 years, the rate is increased to $150 \mathrm{~kg} / \mathrm{s}$. After 30 years $\Delta h=150 \mathrm{~m}$. The next 20 years, the rate is $150 \mathrm{~kg} / \mathrm{s}$, so for this period:

$$
\Delta W_{p}=(20) \text { years }(150) \frac{k g}{s}\left(31.536 \times 10^{6}\right) \frac{\mathrm{s}}{\text { years }}=9.46 \times 10^{10} \mathrm{~kg}
$$

and the drawdown for the period is:

$$
\Delta h_{2}=\frac{\Delta W_{p}}{A \varphi p_{w}+K t_{2}}=\frac{9.46 \times 10^{10} \mathrm{~kg}}{\left(3.9 \times 10^{8}\right) \frac{\mathrm{kg}}{\mathrm{m}}+(0.28) \frac{\mathrm{kg}}{\mathrm{m} \cdot \mathrm{s}}(20)\left(31.536 \times 10^{6}\right) \mathrm{s}}=167 \mathrm{~m}
$$

Therefore the total drawdown after 50 years will be:

$$
\Delta h=150 m+167 m=317 m
$$

\subsection{More Data Points Thain Constants to Fit}

Assume that the following data are available from the reservoir described in the previous example:

Time(days) Rate(kg/s) Drawdown(m) 


\subsubsection{Integrated Form}

Assume that the same model applies as before. By rewriting Eq.3 in an integrated form, the result becomes:

$$
A \varphi \rho_{w} \Delta h_{n}=W_{p n}-K \sum_{j=1}^{n} \Delta h_{j} \Delta t_{j}
$$

dividing by $\sum_{j=1}^{n} \Delta h_{j} \Delta t_{j}$ and rearranging gives:

$$
\frac{W_{p n}}{\sum_{j=1}^{n} \Delta h_{j} \Delta t_{j}}=A \varphi \rho_{w} \frac{\Delta h_{n}}{\sum_{j=1}^{n} \Delta h_{j} \Delta t_{j}}+K
$$

Now defining:

$$
x_{n}=\frac{\Delta h_{n}}{\sum_{j=1}^{n} \Delta h_{j} \Delta t_{j}}
$$

and

$$
y_{n}=\frac{W_{p n}}{\sum_{j=1}^{n} \Delta h_{j} \Delta t_{j}}
$$

Substituting, the relationship becomes:

$$
y_{n}=A \varphi p_{w} x_{n}+K
$$

This equation may be used to identify the constants $A \varphi \rho_{w}$ and K. There are several ways to do that. One is using a least squares fitting technique. The purpose is to minimize the distance between the data points and the points calculated using an equation of the form given above. The data points can also be graphed on cartesian graph paper. In this case the line should be straight. This is always recommended if the equations can be reduced to an equation of a 
straight line on some form of graph paper. If the points fall on a straight line, the model used is likely to apply. The scatter of data points may be due to the sensitivity of the method. It does make a difference how the equations are written. If dividing by $\Delta h$ rather than the sum, $W_{p n} / \Delta h_{n}$ is close to being constant. Writing the equation of a straight line like that would therefore not be a good choice.

Returning to the example, the values of $x_{n}$ and $y_{n}$ were calculated. The results of the calculations are given in Table 2. A graph of the data points is given in Fig.14. As can be seen from this graph, the points fall on a straight line. The solid line represents the least squares fit through the data. The two constants from the fit are the slope and the intercept with the ordinate. The values are:

$A \varphi \rho_{\omega}=4.03 \times 10^{8} \mathrm{~kg} / \mathrm{m}$

$\mathrm{K}=0.3 \mathrm{~kg} / \mathrm{m} \mathrm{s}$

$K$ becomes inaccurate if the points are scattered, because the value of $K$ is small. If the uncertainty in measurements is high, it may be necessary to smooth the data using statistical methods.

If there are more than two coefficients to determine, it is not possible to write the equations in the simple straight line form, and a numerical method is necessary. The general form of writing equations with constant coefficients is:

$$
y_{j}=a_{0}+a_{1} x_{1, j}+a_{2} x_{2, j}+\ldots .+a_{m} x_{m, j}
$$

In matrix form this becomes:

$$
\left[\begin{array}{ccccc}
1 & x_{1,1} & x_{2,1} & \cdots & x_{m, 1} \\
\vdots & \vdots & \vdots & & \vdots \\
i & x_{1, n} & x_{2, n} & \cdots & x_{m, n}
\end{array}\right] \times\left[\begin{array}{c}
a_{0} \\
\cdot \\
\cdot \\
a_{m}
\end{array}\right]=\left[\begin{array}{c}
y_{1} \\
\vdots \\
y_{n}
\end{array}\right]
$$

If $m=n$, there are the same number of data points as constants to fit, and the matrix equation can be solved. If $m>n$, there is not a unique solution. If $m<n$, 
there are more data points than constants, and the least squares method must be used.

\subsubsection{Finite Difference Form}

Previously Eq. 3 was written in an integrated form, assuming $A \varphi \rho_{w}$ and $\mathrm{K}$ constant. Eq. 3 could also be written in a finite difference form. There are several ways of doing this, many of which are used in numerical simulation. One way would be:

$$
A \varphi \rho_{w} \frac{h_{n}-h_{n-1}}{t_{n}-t_{n-1}}=K \Delta h_{n}-w_{n}
$$

Note

that

$\Delta h_{n}=h_{i}-h_{n} \quad$ implies

that

$h_{n}-h_{n-1}=\left(h_{i}-h_{n-1}\right)-\left(h_{i}-h_{n}\right)=\Delta h_{n-1}-\Delta h_{n}$. Dividing by $\Delta h_{n}$ gives:

$$
A \varphi \rho_{w} \frac{\Delta h_{n-1}-\Delta h_{n}}{\Delta h_{n} \Delta t_{n}}=K-\frac{w_{n}}{\Delta h_{n}}
$$

where $\Delta t_{n}=t_{n}-t_{n-1}$. Rearranging gives:

$$
\frac{w_{n}}{\Delta h_{n}}=K+A \varphi \rho_{w} \frac{\Delta h_{n}-\Delta h_{n-1}}{\Delta h_{n} \Delta t_{n}}
$$

and by defining $y_{n}=\frac{w_{n}}{\Delta h_{n}}$ and $x_{n}=\frac{\Delta h_{n}-\Delta h_{n-1}}{\Delta h_{n} \Delta t_{n}}$, a similar straight line relationship results.

Knowing $A \varphi \rho_{w}$, the drawdown after 2000 days of production, if the rate is $110 \mathrm{~kg} / \mathrm{s}$ after 1000 days, can be predicted.

The equation is:

$$
A \varphi p_{w} \Delta h_{n}=W_{p n}-K \sum_{j=1}^{n} \Delta h_{j} \Delta t_{j}=W_{p n}-K \sum_{j=1}^{n-1} \Delta h_{j} \Delta t_{j}-K \Delta h_{n} \Delta t_{n}
$$

Solving for $\Delta h_{n}$ gives:

$$
\Delta h_{n}=\frac{W_{p n}-K \sum_{j=1}^{n-1} \Delta h_{j} \Delta t_{j}}{A \varphi \rho_{w}+K \Delta t_{n}}
$$


From Table 2 the sum is known, and the other terms are known:

$$
\begin{aligned}
W_{p n}=W_{p n-1}+\Delta W_{p n}=7.344 \times 10^{9} \mathrm{~kg} & +\left(86.4 \times 10^{6}\right) \mathrm{s}(110) \frac{\mathrm{kg}}{\mathrm{s}}=16.85 \times 10^{9} \mathrm{~kg} \\
K & =0.3 \frac{\mathrm{kg}}{\mathrm{ms}} \\
A \varphi \rho_{w} & =4.03 \times 10^{8} \frac{\mathrm{kg}}{\mathrm{m}} \\
\Delta t_{n} & =86.4 \times 10^{6} \mathrm{~s} \\
\sum_{j=1}^{n-1} \Delta h_{j} \Delta t_{j} & =841.1 \times 10^{6} \mathrm{~ms}
\end{aligned}
$$

Substituting these values gives the drawdown after 2000 days:

$$
\Delta h_{n}=\frac{16.85 \times 10^{9} \mathrm{~kg}-(0.3) \frac{\mathrm{kg}}{\mathrm{ms}}\left(841.1 \times 10^{6}\right) \mathrm{ms}}{\left(4.03 \times 10^{8}\right) \frac{\mathrm{kg}}{\mathrm{m}}+(0.3) \frac{\mathrm{kg}}{\mathrm{ms}}\left(86.4 \times 10^{6}\right) \mathrm{s}}=38.7 \mathrm{~m}
$$

In all the calculations the simplest possible way of numerically integrate the influx equation have been used. It should be noted that this introduces an error in the determination of the influx constant. Using the average drawdown between the current and the present level of depletion in calculating the sum would approximate the integral better. However, with the number of time steps, and the frequency of values when the drawdown changes mast rapidly; the formulation used above gives a good approximation. The additional terms added to each depletion step are also nearly constant, so even though the influx constant may not be the true Schilthuis constant, the predicted drawdown should be the same.

\subsection{Determination of Optimum Constants}

$U_{p}$ to this point cases have been considered where the constants could be obtained through a least squares fitting technique. Some constants can not be obtained in this manner, and other method must be used. Some of the most important constants to identify, are the initial values of the parameters. This is important when the variations of saturation, temperature, and pressure need to be 
described with time. Initially, there may be only liquid, but the drawdown may not follow this type of model for a very long time. There will eventually be boiling in the reservoir, and to be able to predict when the pressure falls on the saturation curve, the initial pressure in the reservoir must be known. As an example, the simplified mass balance given in Eq.9 with Schilthuis influx can be used:

$$
V \varphi\left(\rho_{t i}-\rho_{t n}\right)=W_{p}-K \sum_{j=1}^{n} \Delta h_{j} \Delta t_{j}
$$

In this case the initial density needs to be identifed, which is a function of the initial thermodynamic properties in the reservoir. The initial temperature is usually well known, therefore assume that the only initial parameter to fit is the initial pressure. If there are more than one parameter to fit, they can be fitted one at a time, and iterating until-convergence. The procedure is as follows:

1. Assume initial pressure $p_{i}$.

2. Calculate the corresponding $\rho_{t i}$. (There may be steam present, or the fluid is on the saturation curve, depending on the temperature and the chemical composition of the fluid.)

3. Perform the history match as discussed in the previous section.

4. Determine the standard deviation from the fit:

$$
\text { s.d. }=\sqrt{\frac{\sum \Delta^{2}}{m-1}}
$$

where

$\Delta=y_{n}-y_{n}^{\prime}$

$y_{n}$ is from the data

$y_{n}^{\prime}$ is calculated using the fit, and

$m=$ the number of data points.

5. Change the value of $p_{i}$, and repeat from step 2 . 
When the procedure has been finished for several values of $p_{i}$, the assumed values of $p_{i}$, vs. the standard deviation can be graphed. If there is a minimum s.d., the corresponding initial pressure is assumed to be the correct value. The same procedure may be used to identify other constants. 


\section{EARIIER SVARTSENGI MODEIS}

\subsection{Reservoir Description}

The Svartsengi field in Iceland is a liquid dominated reservoir with fluids of nearly constant temperature at $235^{\circ} \mathrm{C}$. The reservoir is located on Reykjanes Peninsula in southwest Iceland. From resistivity measurements the reservoir is believed to cover an area of $5 \mathrm{~km}^{2}$ at $200 \mathrm{~m}$ depth, increasing to $7 \mathrm{~km}^{2}$ at $600 \mathrm{~m}$ below sea level. The salinity of the geothermal fluid is about two thirds that of sea water.

The following reservoir and fluid properties were discussed by Regalado. ${ }^{23}$ Fluid production from the reservoir started in 1976. The production data have been provided by Thorhallsson. ${ }^{30}$ The total mass rate output from the field is shown in Fig.15a. The water level was measured in wells 5 and 6 until 1000 days of production. After 1200 days of production, the drawdown was measured in well 4. The measured drawdown vs. time is given in Fig.15b. A pressuretemperature diagram for the wells is shown in Fig.16. It shows the feed zone pressures of the wells and the water saturation temperature corresponding to the pressure profile measured in well 4 . Note that only well 3 is initially at saturated conditions, and that the temperature from $350 \mathrm{~m}$ to $1650 \mathrm{~m}$ depth is nearly constant. Most of the wells are completed in the deeper liquid zone. The pressure with depth is linearly increasing, but there was initially a 16 bar pressure difference between the inside and the outside of the reservoir at $1000 \mathrm{~m}$ depth. The density was measured as nearly constant at $854 \mathrm{~kg} / \mathrm{m}^{3}$. The result of enthalpy measurements in wells $7,8,9,10$, and 11 , show average enthalpy of 1074 $\mathrm{kJ} / \mathrm{kg}$. From well testing the porosity and permeability have been reported as 0.1 and 1 darcy, respectively. This permeability appears rather high compared to other liquid dominated geothermal reservoirs. 


\subsection{Convection Model}

The deuterium and chloride concentrations of the reservoir fluid give conflicting information about the relative amounts of fresh and seawater at Svartsengi. The deuterium indicates $50 \%$ meteoric water and $50 \%$ seawater while the chloride indicates $1 / 3$ meteoric and $2 / 3$ sea water. This problem was addressed in the convection model developed by Eliasson ${ }^{24}$ and reported by Regala$\mathrm{do}^{23}$ and Kjaran ${ }^{25}$ where boiling was assumed to explain the high salinity. Also since the temperature in the reservoir is almost constant below $350 \mathrm{~m}$ depth, convection was assumed to occur. It can be shown that a vertical permeability of only about 1 millidarcy is a sufficient condition for convection. ${ }^{26}$ A convection model was therefore proposed for the Svartsengi reservoir. ${ }^{25}$ This natural state model is illustrated in Fig.17. The conservation equations for points $A$ and $B$ can be written as follows:

\section{Point A:}

Mass: $w_{u}=w_{c}+w_{d}+w_{s}$

Energy: $w_{u} h_{u}=w_{s} h_{s}+w_{d} h_{d}+w_{c} h_{c}$

Concentration: $w_{u} \beta=w_{d} \gamma+w_{c} \gamma$

Point B:

Mass: $w_{u}=w_{c}+w_{b}$

Energy: $W_{u} h_{u}=w_{c} h_{c}+w_{b} h_{b}$

Concentration: $w_{u} \beta=w_{c} \gamma+w_{b} \alpha$

Rearranging these equations we get the expressions for the mass flowrates:

$$
\begin{aligned}
w_{c} & =w_{u} \frac{\beta-\alpha}{\gamma-\alpha} \\
w_{b} & =w_{u} \frac{\gamma-\beta}{\gamma-\alpha} \\
w_{d} & =w_{u} \frac{\alpha}{\gamma} \frac{\gamma-\beta}{\gamma-\alpha}
\end{aligned}
$$




$$
\begin{aligned}
& w_{s}=w_{u} \frac{\gamma-\beta}{\gamma} \\
& h_{c}=h_{u}-L \frac{\gamma-\beta}{\gamma} \\
& h_{b}=h_{u}+L \frac{\beta-\alpha}{\gamma}
\end{aligned}
$$

where $L$ is the latent heat of vaporization, and $\alpha, \beta, \gamma$, are the chloride concentrations at the points given in Fig.17. There are now six equations with twelve unknowns. To solve them it becomes necessary to make some assumptions. Assuming that the chloride concentrations are known, the unknowns are limited to seven. The problem can be solved, for example, if the mass flowrate up can be estimated. This was done by using the following expression: ${ }^{2 \theta}$

$$
w_{u}=K A(1-\varepsilon)\left(\rho\left(T_{\varepsilon}, \gamma\right)-\rho\left(T_{u}, \beta\right)\right)
$$

where $K$ is the coefficient of permeability, $\mathrm{m} / \mathrm{s}, A$ is the area of the up flow zone, and $\varepsilon$ is the fraction of the energy disipation which occurs in the down flow (must be smaller than 0.5).

The natural heat loss was calculated as the difference between the upward and the downward heat flow. The value arrived at by Kjaran et al. ${ }^{24}$ was 300 MWthermal. This model describes the reservoir in its natural state.

\subsection{Hydrological Model}

The early production model developed by Kjaran et al. ${ }^{24}$ for the Svartsengi field was a hydrological model. It was based on a rectangular geometry of the fleld where the wells were assumed to be close to one end of the rectangle. The opposite boundary was assumed to be far enough away so the effects of it could be ignored. Writing the boundary value equation for fluid flow in a porous medium lead to the solution:

$$
\Delta h(x, y, t)=\frac{1}{A S} \sum_{n=0}^{\infty} \sum_{m=0}^{\infty} C_{n m} \Phi_{n m}(x, y) \Phi_{n m}(\xi, \eta) \int_{0}^{t} q(\tau) e^{-(t-\tau) / K_{n m}} d \tau
$$

where: 
$A=$ area of the rectangle $\mathrm{m}^{2}$

$S=$ storage coefficient

$\mathrm{T}=$ transmissivity $\mathrm{m}^{2} / \mathrm{s}$

$(x, y)=$ coordinates of observation well, $m$

$(\xi, \eta)=$ coordinates of the producing well, $\mathrm{m}$

$\Delta h(x, y, t)=$ the drawdown in the observation well, $m$

The matrices are given by:

$$
\begin{gathered}
C_{n m}=\left\{\begin{array}{cc}
4 & \begin{array}{c}
n \neq 0, m \neq 0 \\
2
\end{array} \\
1 & (n \neq 0 \Lambda m=0) V(n=0 \Lambda m \neq 0) \\
n=0 \Lambda m=0
\end{array}\right. \\
K_{n m}=\frac{S}{\pi^{2} T\left(\frac{m^{2}}{s^{2}}+\frac{n^{2}}{b^{2}}\right)} \\
\Phi_{n m}=\cos \frac{m \pi x}{a} \cos \frac{n \pi y}{b}
\end{gathered}
$$

This solution corresponds to the solution for a well located in a rectangle. The effects of superimposed wells are added in an infinite array to create the boundaries.

For step changes in the rate, this equation may be written as a superposition sum. The resulting equation was used to calculate the drawdown in the observation well. The result of the calculated drawdown compared to the measured drawdown is given in Fig.18. We see that there is a good agreement during the first 1600 days of exploitation.

\subsection{Unit Response Function Model}

The unit response function was defined by Barelli et al. ${ }^{27}$ as the solution to the diffusivity equation: 


$$
\eta \nabla^{2} p(\vec{r}, t)-\frac{\partial p(\vec{r}, t)}{\partial t}=0
$$

with the boundary conditions along a boundary S:

$$
\begin{aligned}
& p(\vec{r}, t)=\bar{p}(\vec{r}, t) \text { on } S_{1} \\
& p(\vec{r}, 0)=p_{0}(\vec{r}) \text { in } \mathrm{V} \\
& A_{2} \frac{k}{\mu} \Delta p(\vec{r}, t) \cdot \vec{n}=q(t)+q^{\prime}(t) \text { on } S_{2} \\
& \frac{k}{\mu} \Delta p(\vec{r}, t) \cdot \vec{n}=v(\vec{r}, t) \text { on } S_{3}
\end{aligned}
$$

In these equations, $\eta$ is the diffusivity $\left(\mathrm{m}^{2} \mathrm{~s}^{-1}\right), \mathrm{v}$ is the Darcy velocity, and $q^{\prime}(t)$ is the flow rate variation on $S_{2}$. $S_{1}$ is the part of the boundary on which pressure is known, $S_{2}$ represents the well where the rate variation takes place, and the rate is known on $S_{\mathrm{g}}$. The solutions to $\mathrm{Eq} .43$ are presented for some boundary conditions in Table 1. However, a more general solution may be obtained without putting further restrictions on the boundaries than what is stated above. If allowing the flowrate to change stepwise, the solution becomes: 27

$$
p_{d}(\vec{r}, t)=q(0+) p_{r}(\vec{r}, t)+\int_{0}^{t} p_{r}(\vec{r}, \tau) \frac{d g(t-\tau)}{d t} d \tau
$$

where $p_{r}\left(P a m^{-3} s\right)$ is the special unit response function, which is the solution to the boundary condition of step changes in rate, and $p_{d}(P a)$ is the pressure field difference between disturbed and undisturbed solution. ${ }^{27}$

Eq.44 can be rewritten for the step changes in rate using the superposition sum:

$$
\Delta h=q(0+) F(t)+\sum_{j=1}^{n} \Delta q_{j} F\left(t_{n}-t_{j}\right)
$$

Now the problem becomes to determine the unit response function $F(t)$. The unit response function has been used to model the Svartsengi field. ${ }^{23}$ The function $F(t)$ was determined using a least squares fitting routine with the drawdown history in the reservoir. The calculated drawdown is shown in Fig.19. The unit 
response function is determined using the measured drawdown and the rate variations in the field. The solution is general, and any function which fits the data may be used. When $F(t)$ has been determined, conventional well testing techniques may be used to determine permeability, storage, and skin, by log-log type curve matching.

\subsection{Linear Model}

A linear model developed by Swedish authors was used for the Svartsengi reservoir by Regalado. ${ }^{23}$ The model was developed for a long flow channel called an esker that forms under glaciers. Regalado ${ }^{23}$ adopted this model because geothermal fields are geologically active with many parallel faults. The diffusivity equation was written:

$$
\frac{\partial^{2} h}{\partial x^{2}}=\frac{S}{T} \frac{\partial h}{\partial t}
$$

and solved with the boundary conditions:

$$
\begin{gathered}
h(x, 0)=h(\infty, t)=h_{0} \\
\frac{\partial h}{\partial x}(0, t)=-\frac{q}{2 T b}
\end{gathered}
$$

In these equations $T$ is the transmissivity $\left(\mathrm{m}^{2} / \mathrm{s}\right)$, and $S$ is the storativity coefficient. The initial condition, and the first boundary condition say that the initial pressure is the same as the pressure at the infinite distance from the well. The second boundary condition is Darcy's law written at the producing well positioned at $x=0$. This well is considered a plane source, and the observation well is located a distance $\mathbf{x}$ from this source. The model assumes infinite linear aquifer, but the reservoir is assumed to be closed at one end. To create this boundary, an additional production well, producing at a rate q, was placed at a distance 21-x from the observation well. This well was referred to as the image well. The model is illustrated on Fig.20. The solution to this problem becomes:

$$
\Delta h=\frac{1}{2 \sqrt{\pi}} \frac{q(x)}{T b} \cdot D\left(w_{1}\right)+\frac{1}{2 \sqrt{\pi}} \frac{q(21-x)}{T b} D\left(w_{2}\right)
$$


where:

$$
\begin{gathered}
w_{1}=\frac{x^{2} S}{4 T t} \\
w_{2}=\frac{(21-x)^{2} S}{4 T t} \\
D(w)=\frac{e^{-w}}{\sqrt{w}}-\sqrt{\pi}+2 \int_{0}^{\sqrt{w}} e^{-z^{2}} d z
\end{gathered}
$$

The first term in Eq.48 is the drawdown due to the production well, and the second term is due to the image well. This model is the same as a semi-infinite aquifer with the superimposed effect of the image well to create the no flow boundary. The model was used to calculate the drawdown for the first 1600 days of production using relevant reservoir dimensions, properties, and flowrate data. The results are given in Fig.21. The model agrees well with the observed drawdown in the field.

\subsection{Boiling Model}

The pressure-temperature diagram in Fig.16 shows that drawdown in the field will result in boiling in the upper parts of the reservoir. As the liquid level drops, there will be a boiling zone above the liquid level where the pressure and the temperature follow the boiling point with depth curve. This has been observed at Svartsengi, where steam is now rising from the ground in the region of the shallow wells. A model was developed by Kjaran et al. ${ }^{28}$ using the storativity given by:

$$
S_{1}=\varphi\left(1-S_{w}\right)
$$

where the subscript 1 referres to the zone where there is boiling. This quantity is assumed constant. The total production rate is given by the sum of the rate from the lower liquid zone, and the rate from the two phase zone:

$$
\begin{gathered}
w_{2}=w_{1}+\rho_{2} A_{2} S_{2} \frac{d h_{2}}{d t} \\
w_{1}=\rho_{1} A_{1} S_{1} \frac{d h_{1}}{d t}
\end{gathered}
$$


where $\mathrm{h}$ is the drawdown in meters, and the subscript 2 referres to the liquid zone deeper in the reservoir. These equations are similar to the unconfined system with the influx term replaced by $w_{1}$; the rate from the two phase zone. Furthermore, it was assumed that the rate from the two phase zone was proportional to the difference in pressure between the two zones:

$$
w_{1}=c\left(h_{2}-h_{1}\right)
$$

There are now three equations with three unknowns, $w_{1}, h_{1}$, and $h_{2}$. The drawdown in the deep zone, $h_{2}$, is of interest since this is what is monitored in the observation well. The solution to these equations is:

$$
h_{2}(t)=C_{1} W_{p}(t)-C_{2} \int_{0}^{t} W_{p}(t-\tau) e^{-T / K} d \tau
$$

where:

$$
\begin{gathered}
C_{1}=\frac{1}{\rho_{2} A_{2} S_{2}} \\
C_{2}=c C_{1}^{2} \\
K=\frac{1}{c} \frac{\left(\rho_{1} A_{1} S_{1}\right)\left(\rho_{2} A_{2} S_{2}\right)}{\rho_{1} A_{1} S_{1}+\rho_{2} A_{2} S_{2}}
\end{gathered}
$$

It is also possible to calculate the drawdown in the two phase zone:

$$
h_{1}(t)=C_{3} \int_{0}^{t} w_{p}(t-\tau) e^{-\tau / K} d \tau
$$

where:

$$
c_{9}=\frac{c}{\left(\rho_{1} A_{1} S_{1}\right)\left(\rho_{2} A_{2} S_{2}\right)}
$$

The constants in Eq.56 were reported as being: ${ }^{2 \theta}$.

$C_{1}=1.488 \times 10^{-8} \mathrm{~m} / \mathrm{kg}$

$C_{2}=8.716 \times 10^{-11} \mathrm{~m} / \mathrm{kg}$ day 
$K=150$ days.

To use this model, the equations must be numerically integrated. The drawdown was calculated here using the constants given above and the following equation:

$$
\Delta h_{n}=1.488 \times 10^{-8} W_{p n}-8.716 \times 10^{-11} \sum_{j=1}^{n} W_{p}\left(t_{n}-t_{j}\right) e^{-t_{j} / 150} \Delta t_{j}
$$

This equation is the same as Eq.56 for $h_{2}$ but is now written in numerically integrated form. The results of the calculations are given in Fig.22. The fit is not very good, although the shape of the curve is similar to the data. $K$ was changed and history matching was performed to get a better match. $\mathrm{K}$ was determined by trial and error, but $C_{1}$ and $C_{2}$ were determined using a least squares method similar to the example presented in the chapter on history matching. The best match is given in Fig.23. The constants used were:

$C_{1}=1.129 \times 10^{-8} \mathrm{~m} / \mathrm{kg}$

$C_{2}=4.932 \times 10^{-11} \mathrm{~m} / \mathrm{kg}$ day

$K=250$ days

This model is similar to the Schilthuis model, exept in this case the pressure support is from the two-phase zone rather than from a supporting aquifer. From the formulation of the problem, there is no way to distinguish between these two cases. The drawdown in the two-phase region could just as well be representing drawdown in an aquifer. In the Schilthuis method there is no drawdown in the aquifer, the external pressure is always initial pressure. 


\section{DEPLETION ANALYSIS FOR SVARTSENGI}

A spectrum of depletion models have now been developed or modified. The data from Svartsengi were analyzed using these depletion models. In the analysis the simplest models are considered first.

\subsection{Liquid Models}

\subsubsection{Without Recharge}

The production data for the Svartsengi field are given in Appendix B. After producing $3 \times 10^{10} \mathrm{~kg}$ of fluids from the reservoir, the drawdown was measured about $100 \mathrm{~m}$. Eq. 2 and Eq. 6 for for unconfined and confined, respectively, can be used to identify the kind of reservoir. At reservoir conditions, the compressibility of the liquid water is about $1.2 \times 10^{-8} \mathrm{~Pa}^{-1}$. Using the reported values for porosity $(0.1)$ and density $\left(850 \mathrm{~kg} / \mathrm{m}^{3}\right)$, the volume of the reservoir can be determined from Eq.6 if the production is due to decompression ; a confined system. Integrating Eq. 6 with $W_{e}=0$ gives:

$$
V \varphi \rho_{w}^{2} g c_{t w} \Delta h=W_{p}
$$

Solving for $\mathrm{V}$ gives:

$$
V=\frac{W_{p}}{\varphi \rho_{w}^{2} g c_{t w} \Delta h}
$$

where $c_{t w}=c_{w}+c_{\sigma}$. Assume that $c_{\sigma}=1.15 \times 10^{-9} \mathrm{~Pa}^{-1}$. Then $c_{t w}=2.35 \times 10^{-9} \mathrm{~Pa}^{-1}$. Substituting the volume becomes:

$$
V=\frac{3 \times 10^{10} \mathrm{~kg}}{(0.1)(850)^{2}\left(\mathrm{~kg} / \mathrm{m}^{3}\right)^{2}(9.81) \mathrm{m} / \sec ^{2}\left(2.35 \times 10^{-9}\right) \mathrm{Pa}^{-1}(100) \mathrm{m}}=180 \times 10^{9} \mathrm{~m}^{3}
$$

If assuming that the area, as determined from the resistivity measurements, is about $7 \mathrm{~km}^{2}$, the height of the reservoir should be:

$$
h_{i}=\frac{V}{A}=\frac{180 \times 10^{9} \mathrm{~m}^{3}}{7 \times 10^{6} \mathrm{~m}^{2}}=26 \mathrm{~km}
$$

This height is impossibly large. It can therefore be concluded that unless the area is much larger than assumed, the model is unlikely to apply. However, it is 
possible that the production was supported by a large aquifer outside the reservoir. In that case the reservoir and aquifer must be considered as one unit.

Next, assume that the reservoir is like a completely open tank; an unconfined system. Eq.2 can then be used. Solving for the surface or lateral area:

$$
A=\frac{W_{p}}{\varphi \rho_{w} \Delta h}=\frac{3 \times 10^{10}}{(0.1)(850) \mathrm{kg} / \mathrm{m}^{3}(100) \mathrm{m}}=3.5 \times 10^{6} \mathrm{~m}^{2}
$$

This value of $3.5 \mathrm{~km}^{2}$ is more realistic since it is about half the measured surface area. The value of the porosity is uncertain. With a porosity of $5 \%$ the area would be calculated as $7 \mathrm{~km}^{2}$ by Eq.2, which is the value expected from resistivity measurements. Therefore, the unconfined model appears to be applicable. Note that if there is recharge to the reservoir then $\Delta h$ will be smaller. So the area calculated by Eq. 2 will be less. The drawdown without recharge would be larger.

Graphing $W_{p}$ vs. $\Delta h$ should give a straight line if there is no water recharge or recharge. Fig. 24 shows that the drawdown vs. production is not a straight line indicating recharge. Also notice the close relationship between the rate and the drawdown in Fig.15a and Fig.15b.

The pressure measured in the observation well is not necessarily representative of the average pressure in the reservoir. There may be interference from the producing wells around the observation well, causing the pressure to appear lower. To get the true average pressure, the reservoir should be shut in and allowing the pressure to stabilize. This is impossible since the reservoir is continually producing. To include the effects from each well, a superposition of the effects from all the wells would be necessary. However, in all the models presented in this report, the measured pressure is assumed to be representative for the reservoir. 


\section{- 7.1.2. With Recharge}

\subsubsection{Schilthuis Method}

This model was presented in the section on history matching. When graphing:

$$
x_{n}=\frac{\Delta h_{n}}{\sum_{j=1}^{n} \Delta h_{j} \Delta t_{j}}
$$

vs.

$$
y_{n}=\frac{W_{p n}}{\sum_{j=1}^{n} \Delta h_{j} \Delta t_{j}}
$$

the points on Fig.25 were obtained. The first few points are distinctly larger in magnitude than the rest of the points. The solid line in Fig.25 represents the least squares fit using all the points. The constants from the fit are:

$a_{0}=3.04377 \mathrm{~kg} / \mathrm{m} \mathrm{s}$

and

$a_{1}=5.33611 \times 10^{7} \mathrm{~kg} / \mathrm{m}$

The group of terms in front of $\mathrm{dh} / \mathrm{dt}$ in Eq. 2 and Eq. 6 is a constant. Define this constant to be $S_{W}$, the mass storativity coefficient. Thus, for the unconfined case:

$$
S_{\eta}=A \varphi \rho_{w}
$$

and for the confined case:

$$
S_{\nabla}=V \varphi \rho_{w}^{2} g c_{t w}
$$

The equations for the confined and unconfined models now have the same form. For The Schilthuis model $S_{\eta}=a_{1}$, and $K=a_{0}$. Assuming an unconfined system:

$$
a_{1}=A \varphi \rho_{w}
$$

and 


$$
A=\frac{a_{1}}{\varphi \rho_{w}}=\frac{5.33611 \times 10^{7} \mathrm{~kg} / \mathrm{m}}{(0.1)(850) \mathrm{kg} / \mathrm{m}^{3}}=0.63 \times 10^{6} \mathrm{~m}^{2}
$$

For a confined system, however, the volume is given by:

$V=\frac{a_{1}}{\varphi \rho_{w}^{2} g c_{t w}}=\frac{5.33611 \times 10^{7} \mathrm{~kg} / \mathrm{m}}{(0.1)(840)^{2}\left(\mathrm{~kg} / \mathrm{m}^{3}\right)^{2}(9.81) \mathrm{m} / \mathrm{sec}^{2}\left(2.35 \times 10^{-9}\right) P a^{-1}}=3.2 \times 10^{10} \mathrm{~m}^{3}$ Assuming that the area is $7 \mathrm{~km}^{2}$, the height of the reservoir is:

$$
h_{i}=\frac{V}{A}=\frac{3.2 \times 10^{10} \mathrm{~m}^{3}}{7 \times 10^{6} \mathrm{~m}^{2}}=4.6 \mathrm{~km}
$$

The drawdown was calculated using Eq.40. The result is given in Fig.26. The solid line represents the calculated values, the circles are the measured values. This plot is the same for the confined and the unconfined system. The difference between the two is in the formulation of $S_{W}$.

The real system seems to behave somewhere in between these cases. Notice also that the fit is good for the early data, but the influx is too strong for the later part of the data. If the three first points are excluded from the fit, we get a slightly different fit. The fit is given in Fig.27, and the drawdown in Fig.28. We see that the influx is not as strong in this case. From the least squares fit, $\mathrm{K}=2.75447$. The value for $S_{N}$ is $6.60567 \times 10^{7}$. The influx is however still too strong, although this fit is better.

Consider what information can be obtained from the Schilthuis influx constant K. From Eq.14 for the linear case $p=\rho_{w} g h$ :

$$
K=\frac{k A \rho_{w}^{2} g}{\mu \Delta L}
$$

In the radial case Darcy's law gives:

$$
K=\frac{2 \pi k h \rho_{w}^{2} g}{\mu \ln \frac{r_{g}}{r_{w}}} \frac{\theta}{360}
$$

where $\theta$ is the angle open to flow. In the linear case the length of the aquifer can be obtained from the first equation using $k \sim 1$ darcy: 
$\Delta L=\frac{k A \rho_{w}^{2} g}{\mu K}=\frac{\left(1 \times 10^{-12}\right) \mathrm{m}^{2}(2500) \mathrm{m}(1500) \mathrm{m}(850)^{2}\left(\mathrm{~kg} / \mathrm{m}^{9}\right)^{2}(9.81) \mathrm{m} / \mathrm{s}^{2}}{\left(110 \times 10^{-6}\right) \mathrm{Pa} \cdot \mathrm{s}(3) \mathrm{kg} / \mathrm{m} \cdot \mathrm{s}}=80 \mathrm{~km}$ In this equation $A$ is the cross-sectional area of the aquifer. This was taken as $2500 \mathrm{~m} \times 1500 \mathrm{~m}$, since $2500^{2} \mathrm{~m}^{2}=6.25 \mathrm{~km}^{2}$, and $1500 \mathrm{~m}$ is a reasonable height. The distance from the reservoir to the ocean, which may perhaps act as a constant pressure outer boundary, is about $20 \mathrm{~km}$. The cross-sectional area of the aquifer may be less than what was used and the permeability of the aquifer is likely to be much less. The calculated length is therefore of reasonable magnitude. Assuming radial geometry, $\theta$ can be determined from the second equation. Assuming $r_{e} / r_{w}=10$ :

$$
\begin{gathered}
\theta=\frac{360^{\circ} K \mu \ln \frac{r_{e}}{r_{w}}}{2 \pi k h \rho_{w}^{2} g} \\
\theta=\frac{\left(360^{\circ}\right)(3) \mathrm{kg} / \mathrm{m} \cdot \mathrm{s}\left(110 \times 10^{-\theta}\right) \mathrm{Pa} \cdot \mathrm{s} \ln 10}{2 \pi\left(1 \times 10^{-12}\right) \mathrm{m}^{2}(1500) \mathrm{m}(850)^{2}\left(\mathrm{~kg} / \mathrm{m}^{9}\right)^{2}(9.81) \mathrm{m} / \mathrm{s}^{2}}=4^{\circ}
\end{gathered}
$$

This small angle shows that a linear geometry is more valid than a radial system.

\subsubsection{Hurst Modified Method}

In this method the influx rate is a function of time. The influx rate is given by Eq.21. Combining it with Eq.3, the following results:

$$
\frac{A \varphi}{g} \frac{d p}{d t}=\frac{a\left(p_{i}-p\right)}{\log (b t)}-w
$$

This is for the unconfined system. Integrating, and substituting the mass storativity coefficient:

$$
-S_{\#}=\int_{0}^{t} \frac{a \Delta h}{\log (b t)} d t-w
$$

Notice that $a$ has different meanings in the two equations. Numerically integrating the water influx:

$$
-S_{\sharp} \Delta h_{n}=\sum_{j=1}^{n} \frac{a \Delta h_{j} \Delta t_{j}}{\log \left(b \bar{t}_{j}\right)}-W_{p n}
$$


Assuming that for each step of depletion, the following holds:

$$
K_{j}=\frac{a}{\log \left(b \bar{t}_{j}\right)} \quad, \bar{t}_{j}=\frac{t_{j}+t_{j-1}}{2}
$$

it follows that:

$$
-S_{\Downarrow} \Delta h_{n}=\sum_{j=1}^{n} K_{j} \Delta h_{j} \Delta t_{j}-W_{p n}
$$

Solving for $K_{n}$ gives:

$$
K_{n}=\frac{W_{p n}-S_{\#} \Delta h_{n}-\sum_{j=1}^{n-1} K_{j} \Delta h_{j} \Delta t_{j}}{\Delta h_{n} \Delta t_{n}}
$$

If $S_{n}$ is known, $K_{n}$ can be calculated using the above equation. The problem is to determine the constants $a$ and $b$. Rewriting the equation for $K_{n}$ :

$$
\frac{1}{a} \log b+\frac{1}{a} \log \bar{t}_{n}=\frac{1}{K_{n}}
$$

Now define:

$$
\begin{aligned}
& x_{n}=\log \bar{t}_{n} \\
& y_{n}=\frac{1}{K_{n}}
\end{aligned}
$$

A plot of $x_{n}$ vs. $y_{n}$ should give a straight line, and the constants $b$ and a may be determined from the slope and the intercept.

To illustrate this model, $S_{\#}=5 \times 10^{7} \mathrm{~kg} / \mathrm{m}$, equal to the value for $S_{y}$ found in the Schilthuis method was assumed. The fit for $\frac{1}{a} \log b$ and $\frac{1}{a}$ is given in Fig.29. The solid line is the fit, and the circles are the calculated values. Notice that there is a lot of scatter. The computational procedure must be wrong, or this model does not apply.

The above influx equation was based on the log approximation of the line source solution for infinite radial aquifers. If infinite linear aquifer is assumed, the material balance equation becomes:

$$
-S_{W} \Delta h=B \rho g \sum_{j=2}^{n} F_{1 / 2}\left(t_{D n}-t_{D j}\right) \Delta h_{j}^{\prime}-W_{p n}
$$


- Substituting the definitions of $B$ and $F_{1 / 2}$ :

$$
-S_{\sharp} \Delta h=\varphi \operatorname{cbh} L \rho^{2} g \sum_{j=2}^{n} 2\left(\frac{\left(t_{D n}-t_{D j}\right)}{\pi}\right)^{1 / 2} \Delta^{\prime} h_{j}-W_{p n}
$$

By the definition of $T_{D}(\mathrm{~L}=1)$ :

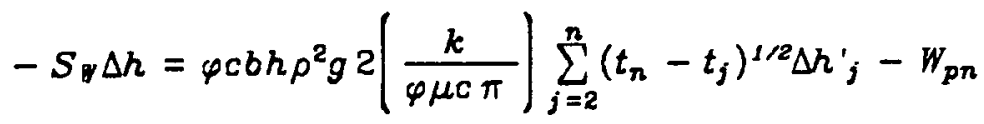

In the above equations, $\Delta h^{\prime}{ }_{j}$ is the pressure difference between each depletion step:

$$
\Delta h^{\prime}{ }_{j}=\Delta h_{j}-\Delta h_{j-1}
$$

Substituting Eq. 64 in Eq. 63 and dividing by $\Delta h_{n}$ :

$$
\begin{aligned}
& \frac{\sum_{j=2}^{n}\left(\Delta h_{j}-\Delta h_{j-1}\right)\left(t_{n}-t_{j-1}\right)^{1 / 2}}{\Delta h_{n}}= \\
& \qquad \frac{1}{\left(\frac{\varphi c k}{\mu \pi}\right)^{1 / 2} b h \rho^{2} g 2}\left(\frac{W_{p n}}{\Delta h_{n}}\right)-\frac{S_{\pi}}{\left(\frac{\varphi c k}{\mu \pi}\right)^{1 / 2} b h \rho^{2} g 2}
\end{aligned}
$$

Eq. 65 can be written in the form:

$$
y_{n}=a_{1} x_{n}+a_{0}
$$

A plot of this match is given in Fig. 30. The points seam to give a positive value of $a_{0}$ which corresponds to a negative value of $S_{y} !$ It is therefore concluded that $S_{\eta}$ is zero, and that the reservoir behaves as infinite linear. With $S_{\#}=0$, the slope is:

$$
a_{1}=1.05 \times 10^{-7} \mathrm{~s}^{1 / 2} \mathrm{~m} / \mathrm{kg}
$$

With $S_{\eta}=0$, Eq. 63 becomes:

$$
W_{p n}=\frac{1}{a_{1}}\left[\sum_{j=2}^{n-1}\left(t_{n}-t_{j-1}\right)^{1 / 2}\left(\Delta h_{j}-\Delta h_{j-1}\right)+\left(\Delta h_{n}-\Delta h_{n-1}\right)\left(t_{n}-t_{n-1}\right)^{1 / 2}\right]
$$

Solving for $\Delta h_{n}$ :

$$
\Delta h_{n}=\Delta h_{n-1}+\left[\frac{a_{1} W_{p n}-\sum_{j=2}^{n-1}\left(\Delta h_{j}-\Delta h_{j-1}\right)\left(t_{n}-t_{j-1}\right)^{1 / 2}}{\left(t_{n}-t_{n-1}\right)^{1 / 2}}\right]
$$


and to get started:

$$
\Delta h_{1}=0, \quad \Delta h_{2}=\frac{a_{1} W_{p 2}}{\sqrt{t_{2}}}
$$

The reason for starting the superposition sum at 2 is that in the input file the first entry is 0 , having subscript 1 . When dividing by $\Delta h_{n}$, the solution blows up for $\Delta h_{1}=0$.

The calculated drawdown is shown in Fig. 31. The match is good, but the aquifer response is too high. The cross-sectional area of the aquifer can be determined from the constant $a_{1}$ :

$$
\begin{aligned}
& a_{1}=\frac{1}{\left(\frac{\varphi c k}{\mu \pi}\right)^{1 / 2} b h \rho^{2} g 2} \\
& b h=\frac{1}{\left(\frac{\varphi c k}{\mu \pi}\right)^{1 / 2} a_{1} \rho^{2} g 2}
\end{aligned}
$$

which becomes:

$$
\begin{gathered}
A=\frac{1}{\left(\frac{(0.1)\left(1 \times 10^{-12}\right) \mathrm{m}^{2}\left(2.35 \times 10^{-9}\right) P a^{-1}}{\left(110 \times 10^{-8}\right) P a \cdot s(\pi)}\right)^{1 / 2}\left(1.05 \times 10^{-7}\right) \frac{\sqrt{s} \mathrm{~m}}{k g}(850)^{2}\left(\mathrm{~kg} / \mathrm{m}^{3}\right)^{2}(9.81) \mathrm{m} / \mathrm{s}^{2}(2)(1) \mathrm{m}} \\
A=8.1 \times 10^{8} \mathrm{~m}^{2}=810 \mathrm{~km}^{2}
\end{gathered}
$$

This value is impossibly large.

\subsubsection{Hurst Simplified Method}

The case of infinite linear aquifer will be considered here. The matching procedure becomes simple in this case. From Eq.26, a straight line through the origin should be obtained by plotting:

$$
x_{n}=\sum_{j=0}^{n} \Delta w_{j} M\left[\lambda^{2}\left(t_{D}-t_{D j}\right)\right]
$$

vs.

$$
y_{n}=\Delta h_{n}
$$


with slope:

$$
a=\frac{\mu_{a q} c_{a q}}{k V \rho_{\text {res }} c_{\text {res }} \rho_{\text {res }} g}
$$

By the definition of $\lambda$, a becomes:

$$
a=\frac{\mu_{a q}}{k A \rho_{\alpha q} \rho_{\text {res }} g} \lambda
$$

where $\mathrm{A}$ is now the cross-sectional area of the aquifer $A=h \cdot b$.

The problem then becomes to determine $\lambda$. Again the method of minimizing the standard deviation will be used. The procedure is as follows:

1. Choose a value for $\lambda$.

2. Calculate $x_{n}$ and $y_{n}$.

3. Find a using least squares fit on:

$$
y_{n}=a \cdot x_{n}
$$

4. Calculate s.d.

5. Change the value of $\lambda_{1}$ and repeat from step 2 .

6. Graph the standard deviation as a function of $\lambda$. The minimum standard deviation corresponds to the value of $\lambda$ which gives the best fit.

When the best fit for $a$ and $\lambda$ is obtained, the drawdown is calculated using Eq. 26 .

The result from the fitting for $\lambda$ is given in Fig.32. The s.d. is decreasing to a minimum at $\lambda=1.3 \times 10^{-4} \mathrm{~m}^{-1}$. Notice that for small values, the s.d. is large, but for large values, the s.d. is low almost constant. When $\lambda$ is large the reservoir is not important in the response. This occurs when the compressibility of the reservoir is low, and points in the direction of an unconfined system.

Assuming that the length of the reservoir is $2500 \mathrm{~m}$ :

$$
\lambda \cdot l=\left(1.3 \times 10^{-4}\right) m^{-1}(2500) m=0.33
$$

since $\lambda=\frac{c_{a q} \rho_{a q}}{l \cdot c_{\text {res }} \rho_{\text {res }}}$ 
Because the reservoir is hotter than the aquifer, the reservoir will have higher compressibility. Any two-phase region at the top of the reservoir may be responsible for a higher compressibility. The constant from the fit was:

$$
a=4.755 \times 10^{-10} \frac{\mathrm{m} \cdot \mathrm{s}}{\mathrm{kg}}
$$

Solving for the cross-sectional area of the aquifer gives (assuming $\rho_{\alpha q}=1000$ $\left.\mathrm{kg} / \mathrm{m}^{3}\right)$ :

$$
\begin{gathered}
A=\frac{\mu_{a q} \lambda}{k \rho_{a q} \rho_{\text {res }} g a} \\
A=\frac{\left(110 \times 10^{-6}\right) P a \cdot s\left(1.3 \times 10^{-4}\right) \mathrm{m}^{-1}}{\left(1.0 \times 10^{-12}\right) \mathrm{m}^{2}(850)(1000)\left(\mathrm{kg} / \mathrm{m}^{3}\right)^{2}(9.81) \mathrm{m} / \mathrm{s}^{2}\left(4.755 \times 10^{-10}\right) \mathrm{m} \cdot \mathrm{s} / \mathrm{kg}}=3.6 \times 10^{6} \mathrm{~m}^{2} \\
\text { If } h=1500 \mathrm{~m} \text {, the width becomes: }
\end{gathered}
$$

$$
b=\frac{A}{h}=\frac{3.6 \times 10^{6} \mathrm{~m}^{2}}{1500 \mathrm{~m}}=2400 \mathrm{~m}
$$

This width agrees well with what would be expected. The calculated drawdown is given in Fig.33.

\subsubsection{Fetkovitch Method}

To history match using this method, trial and error has to be used by changing the parameters in the reservoir and the aquifer. Two cases were investigated: linear and radial geometries. The calculated drawdown is shown in Fig.34 for the linear case, and Fig.35 for the radial case. In the linear case, the best fit was obtained with:

$$
S_{W}=V \varphi \rho_{r e s} c_{r e s}=6.6 \frac{\mathrm{kg}}{P a}
$$

Using the same values for $\varphi, c_{\text {res }}$, and $\rho_{\text {res }}$ as before:

$$
V=\frac{6.6}{\varphi \rho_{\text {res }} c_{\text {res }}}=\frac{6.6 \frac{\mathrm{kg}}{\mathrm{Pa}}}{(0.05)(850) \frac{\mathrm{kg}}{\mathrm{m}^{3}}\left(2.35 \times 10^{-9}\right) P a^{-1}}=6.6 \times 10^{7} \mathrm{~m}^{3}
$$

Assuming $h_{i}=1500 \mathrm{~m}$ : 


$$
A=\frac{V}{h_{i}}=\frac{6.6 \times 10^{7} \mathrm{~m}^{3}}{1500 \mathrm{~m}}=4.4 \times 10^{4} \mathrm{~m}^{2}
$$

This is much smaller than expected. The value of $\frac{3 k b h}{\mu L}$ in Eq.32 was found to be $2.846 \times 10^{-8} \frac{m^{3}}{P_{a} \cdot s}$ Using $b=2500 \mathrm{~m}$ and $k=1 \times 10^{-12} \mathrm{~m}^{2}$, the length of the finite aquifer can be calculated:

$$
L=\frac{3 k b h}{2.846 \times 10^{-6} \frac{m^{3}}{P a \cdot s} \mu}=\frac{(3)\left(0.5 \times 10^{-12}\right) m^{2}(2500) m(1500) \mathrm{m}}{\left(2.846 \times 10^{-6}\right) \frac{m^{3}}{P a \cdot s}\left(110 \times 10^{-6}\right) P a \cdot s}=18 \mathrm{~km}
$$

This is approximately the distance to the ocean from Svartsengi along the fault zone. The model predicts too large a drawdown for late times. This may be due to changes in compressibility as a two phase region forms at the top of the reservoir.

The radial fit was not very satisfactory. so the constants from the fit on Fig. 35 were not determined.

\subsection{Two Zone Model}

Rewriting Eq.10 in a numerically integrated form yields:

$$
-\Delta h_{n}=a \sum_{j=1}^{n} \Delta h_{j} \Delta t_{j}+\frac{b}{\rho g} W_{p n}+\frac{c}{\rho g} w_{n}
$$

which can be written as:

$$
y_{n}=a_{1} x_{1, n}+a_{2} x_{2, n}+a_{3} x_{3, n}
$$

$a_{1}, a_{2}$, and $a_{3}$ are then determined using a least squares fit. From the fit these values were:

$$
\begin{aligned}
& a_{1}=a=0.3261 \times 10^{-7} \\
& a_{2}=\frac{b}{\rho g}=-0.1192 \times 10^{-7} \\
& a_{3}=\frac{c}{\rho g}=-0.3537 \times 10^{-1}
\end{aligned}
$$

Using these constants, the drawdown was calculated using Eq.10 in a slightly different form: 


$$
-\Delta h_{n}=a_{1} \sum_{j=1}^{n-1} \Delta h_{j} \Delta t_{j}+a_{2} W_{p n}+a_{3} w_{n}+a_{1} \Delta h_{n} \Delta t_{n}
$$

Solving for $\Delta h_{n}$ gives:

$$
\Delta h_{n}=\frac{a_{1} \sum_{j=1}^{n-1} \Delta h_{j} \Delta t_{j}+a_{2} w_{p n}+a_{3} w_{n}}{-1-a_{1} \Delta t_{n}}
$$

The result is given in Fig.36. The curve has the right trend, but the line is not smooth because of noise in the data. A discussion of these problems is given by Fradkin et al. ${ }^{5}$. A difference form of this equation does not work since the difference $w_{j}-w_{j-1}$ does not adequately describe $d w$. Note also that it may be difficult to identify $a_{3}$ since when the production stabilizes, the rate is close to being constant. The high fluctuation of $w$ (see Fig.15) may be responsible for the noise in the data. Therefore, using the average rate from the beginning to the time when the drawdown is calculated was tried. The difference is minor, and the result is given in Fig.37. In this case, the fit gave:

$$
\begin{gathered}
a_{1}=a=0.2582 \times 10^{-7} \\
a_{2}=\frac{b}{\rho g}=-0.9583 \times 10^{-8} \\
a_{s}=\frac{c}{\rho g}=-0.1396
\end{gathered}
$$

Eq.10 can be used to solve for $A \varphi, K$, and $\tau_{0}$ assuming a value for $S_{w o}$. From Eq.10c it can be seen that the only way $c$ can be negative, is if $\tau_{0}$ is negative. This makes no physical sense. Thus, even if it is possible to solve for $A \varphi$ and $K$ using Eq.10, the match gives erroneous answers. 


\section{DISCUSSION}

In this study several lumped-parameter depletion models have been used to model the production history of the Svartsengi field. In most cases it was possible to match the drawdown history adequately. The question becomes: What information about the reservoir can be obtained from the results.

\subsection{Liquid Models}

In Fig.24, there must have been recharge in the reservoir if $S_{W}$ is constant. This recharge may be from the edges within the reservoir, or there may actually be a channel where water flows into the reservoir. The models are not able to determine where the recharge comes from, but a better match was obtained when influx was included. First, assuming no transients in the reservoir or the aquifer, two models were considered. For a constant pressure outer boundary, there is an instantaneous steady state, and the Schilthuis method is used. For a closed outer boundary, there is an instantaneous pseudo steady state, and the Fetkovitch method is used. The steady state influx was too strong (Fig.28) causing the calculated drawdown to overreact to rate changes. When only the first half of the data is used, a weeker influx is obtained, but the predicted drawdown is too large for late times (see Fig.38). Assuming a finite aquifer with instantaneous pseudo steady state (this corresponds to when the value of $Q_{D}$ becomes constant in Fig.11 and Fig.12), the trend of the drawdown plotted vs. cumulative mass produced will be straight if $S_{\text {in }}$ is constant. But how good is the assump-

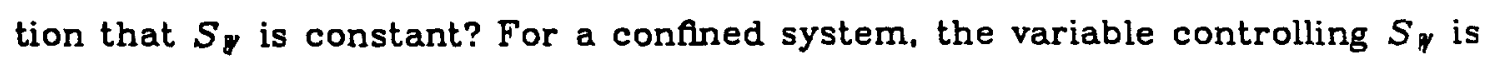
the total compressibility of the system. By the tremendous increase in the total compressibility when boiling occurs, even local boiling can be very important in controlling the compressibility. (See Appendix A for the equations for total compressibility.) This may explain why the calculated drawdown is high for later times. In the case of an unconfined system, this effect may simply be due to an 
increase of the areal extent of the reservoir with depth. The resistivity measurements suggest that the reservoir is only "the tip of the iceberg", connected to a large underlying system.

One way to determine if the Schilthuis model applies, is to try the Hurst modified method for an infinite radial aquifer. If the influx constant is constant with time, the Schilthuis method applies. In this study the Hurst modified method did not improve the match. When an infinite linear aquifer model was used in the Hurst modified method, the match showed $S_{y}=0$. This means that the reservoir and the aquifer are the same. The superposition sum of an infinite aquifer gave a good match with the data, but the variations with rate were too high, leading to the conclusion that there are some effects from the reservoir as well. In order to include these effects, the Hurst simplified method was used. This method gave the best match.

Plotting the $\log$ of drawdown vs. the log of cumulative mass produced gives a straight line. This plot is shown in Fig.39 with a least squares fit represented by the straight line. From the least squares fit, the drawdown becomes:

$$
\Delta h=2.23 \times 10^{-6} W_{p}^{0.732}
$$

In the above equation $W_{p}$ is the cumulative mass in $\mathrm{kg}$, and $\Delta h$ is the drawdown in meters. Using this empirical relationship, the calculated drawdown vs. cumulative mass produced is shown in Fig.40. Considering the straight line in Fig.39, using only the first few points will give almost exactly the same straight line. This model is therefore able to predict the drawdown. The question arises: Why bother going through the complicated depletion models if there is a simple relationship like this one? There are no physical reasons why there should be a log$\log$ relationship between the drawdown and the production. The empirical equation is not a function of rate, and will not be able to predict any build up in the reservoir. 
For comparison of three of the models, the drawdown was predicted using the Hurst simplified method, the boiling model using the best fit discussed earlier, and the empirical $\log -\log$ equation. The result for a rate of $300 \mathrm{~kg} / \mathrm{s}$ is shown in Fig.41. All three models fit the measured data adequately, but the predicted drawdown is very different for the three models. The models should not only match the data, but be physically realistic. The $\log -\log$ method does not take into account a change in the relative amounts of produced fluid and fluid recharge. When the pressure is decreasing, this ratio will change. The empirical model is expected to underestimate the recharge, and it estimates larger drawdown than the two other models. There is also a significant difference between the Hurst simplified and the boiling model. The Hurst simplified method assumes an infinite linear aquifer maintaining the pressure in the reservoir. The boiling model assumes drainage from a two-phase zone without recharge. From the large difference in the predictions it can be concluded that the chose of model makes a difference in predictions.

\section{B.2. Effect of Injection}

In order to maintain pressure in a reservoir, reinjection may be considered. The injected fluid will be colder and will cool down the reservoir. When the volume injected is known, an estimate of the heat depletion in the reservoir can be made. Injection at Svartsengi was discussed by Gudmundsson. ${ }^{29}$ However, some of the injected fluid will break through to wells vie fractures causing production of some of the injected fluid. In depletion modeling the injected fluid must be included in the mass balance:

$$
W_{c}=W_{i}-W_{p}-W_{l}+W_{e}+W_{i n}
$$

Assuming that the injection of cold fluid will not change the compressibility or total density of the system very much, the injection and production terms can be lumped in a net production term: 


$$
W_{p . n t}=W_{p}-W_{i n}
$$

which in differential form becomes:

$$
w_{p, \text { net }}=w_{p}-w_{\text {in }}
$$

Using this, the drawdown for a variety of production schedules can be predicted. As an example, the drawdown using the Hurst simplified method was prediced. The prediction for a constant rate output of $300 \mathrm{~kg} / \mathrm{s}$ is shown in Fig.42, as drawdown vs. cumulative mass produced, and as drawdown vs. time in Fig.43. When reinjection is considered, the net rate will be less. Fig. 44 shows the drawdown vs. time for three different rates. The figure shows that if two thirds of the fluid are reinjected, the pressure will be maintained above the current level for a long time.

It should be noted that no transient effects in the reservoir and changes in temperature, density and compressibility as a result of injecting cold water have been considered.

The natural mass loss due to natural discharge or evaporation has been assumed negligible in all the calculations. This may not be a good approximation. If the rate of mass loss is constant, this error is most pronounced for early time, since that is when the rate was low. 


\section{CONCLUSIONS}

- Lumped parameter models although computationally simple, adequately match drawdown-production data.

- Better results were obtained when influx was included.

- The transient models gave better match than the steady-and pseudosteady state models.

- The best fit was obtained using an infinite linear aquifer model with the Hurst simplified method.

- Determination of constants from the models help to identify the most reasonable model.

- Detection of changes in the mass storativity coefficient may give information about changes in reservoir properties with production.

- Important information about how the reservoir properties change can be obtained when different parts of the data are matched.

- Determination of recharge is possible. 


\section{RECOMMENDATIONS}

The effects of local boiling have been discussed in this report. By including the total compressibility as a variable in the equations, it is possible to estimate the drawdown using the Schilthuis or the Fetkovitch method where the compressibility changes with depletion. The volume of the two-phase zone can be estimated as being the surface area times the drawdown in the reservoir. If there is boiling in this volume, a total compressibility can be calculated using Eq.A-69. Effects of local boiling on mass storativity coefficient could be investigated using this equation.

The total density method discussed in this report allows effects of saturation changes to be included. However, to predict the drawdown using this method, iterative methods must be used. When this method is developed, it will be possible to include effects of a saturation change in the mass balance equation.

For injection studies, including the heat balance will improve the calculations. If the breakthrough time for the fluid is known, the breakthrough of the temperature can be calculated as discussed by Castanier et al. ${ }^{10}$ The effects of injecting cold fluid into the Svartsengi reservoir need to be considered. 


\section{NOMENCLATURE}

A Area of the reservoir or cross-sectional area of the aquifer(m)

$a, b, c \quad$ Coefficients

$b \quad$ Width of aquifer $(\mathrm{m})$

$B \quad$ Van Everdingen and Hurst influx constant $(\mathrm{kg} / \mathrm{Pa})$

C Heat capacity $\left(\mathrm{kJ} / \mathrm{kg} \cdot{ }^{\circ} \mathrm{K}\right)$

c Compressibility $\left(\mathrm{Pa}^{-1}\right)$

$E \quad$ Internal energy $(\mathrm{kJ} / \mathrm{kg})$

erfo Complimentary error function

F Nabor and Barham dimensionless function

g Acceleration constant $\left(\mathrm{m} / \mathrm{s}^{2}\right)$

h Enthalpy $(\mathrm{kJ} / \mathrm{kg})$

$h \quad$ Height of reservoir (m)

$h_{i} \quad$ Initial height of reservoir $(m)$

$\Delta h \quad$ Drawdown $(\mathrm{m})$

I. Modified Bessel function of the first kind of order 0

$I_{1} \quad$ Modified Bessel function of the first kind of order 1

$J \quad$ Productivity index $(\mathrm{kg} / \mathrm{s} \cdot \mathrm{Pa})$

$k \quad$ Permeability $\left(\mathrm{m}^{2}\right)$

$K \quad$ Schilthuis influx constant $(\mathrm{kg} / \mathrm{m} \mathrm{s})$

$K_{0} \quad$ Modified Bessel function of the third kind of order 0

$K_{1} \quad$ Modified Bessel function of the third kind of order 1

$l \quad$ Length of reservoir $(\mathrm{m})$ 
$L \quad$ Length of aquifer (m)

$L^{-1} \quad$ Inverse Laplace transformation

$\boldsymbol{p} \quad$ Pressure $(\mathrm{Pa})$

$\boldsymbol{P}_{D} \quad$ Dimensionless pressure

$\Delta \bar{p} \quad$ Drawdown function in Laplace space

$\bar{p}_{D} \quad$ Dimensionless pressure function in Laplace space

$q \quad$ Volumetric rate $\left(\mathrm{m}^{3} / \mathrm{s}\right)$

$Q \quad$ Cumulative volume $\left(\mathrm{m}^{3}\right)$

$Q_{D} \quad$ Dimensionless cumulative influx

$\bar{Q}_{D} \quad$ Cumulative dimensionless influx function in Laplace space

$\boldsymbol{r} \quad \operatorname{Radius}(\mathrm{m})$

$r_{6} \quad$ External radius

$r_{w} \quad$ Inside radius

$r_{D} \quad$ Dimensionless radius $\left(r_{\mathrm{g}} / r_{w}\right)$

$\boldsymbol{s} \quad$ Variable in Laplace space

s.d. Standard deviation

$S_{w} \quad$ Water saturation (volumetric fraction)

$S_{\text {wo }} \quad$ Irreducable water saturation

$S_{\text {y }} \quad$ Mass storativity coefficient $(\mathrm{kg} / \mathrm{m})$

$t \quad$ Time (s)

$t_{D} \quad$ Dimensionless time

$t_{\dot{D}} \quad$ Variable of integration

$T \quad$ Temperature (K)

$u$ Darcy velocity $(\mathrm{m} / \mathrm{s})$ 


$\begin{array}{ll}V & \text { Volume }\left(\mathrm{m}^{3}\right) \\ w & \text { Mass rate }(\mathrm{kg} / \mathrm{s}) \\ w & \text { Mass }(\mathrm{kg}) \\ \boldsymbol{\tau} & \text { Steam quality (mass fraction of steam) } \\ \mu & \text { Viscosity (Pa s) } \\ \nu & \left.\text { Specific volume ( } \mathrm{m}^{3} / \mathrm{kg}\right) \\ \varphi & \text { Porosity } \\ \rho & \left.\text { Density (kg/m } \mathrm{m}^{3}\right) \\ \tau & \text { Variable of integration } \\ \theta & \text { Angle open to flow in a radial geometry }\end{array}$

\section{SUBSCRIPIS}

$\begin{array}{ll}a q & \text { Aquifer } \\ a v & \text { Average } \\ c & \text { Current } \\ \text { calc } & \text { Calculated } \\ \boldsymbol{e} & \text { Influx } \\ \boldsymbol{i} & \text { Initial } \\ l & \text { Loss } \\ n & \text { Level of depletion } \\ \boldsymbol{p} & \text { Produced } \\ r & \text { Relative (in relative permeamility) } \\ r e s & \text { Reservoir }\end{array}$


s

$\boldsymbol{t}$

$v$

w

$w f$

$\sigma$

$2 p h$
Steam

Total

Constant volume

Liquid water

Well flowing

Rock matrix

Two-phase 


\section{REFERENCES}

1 Whiting,R.L.,Ramey,H.J.Jr,:"Application of Material and Energy Balances to Geothermal. Steam Production," J.Pet.Tech.(July, 1969) 893.

2 Brigham,W.E..Ramey,H.J.Jr,:"Material and Energy Balance in Geothermal Reservoirs." "Reservoir Engineering Assesment of Geothermal Systems," Ramey,H.J.Jr.(editor), Petroleum Engineering Department, Stanford University, (Oct. 1981).

3 Grant,M.A.:"Approximate Calculations Based on a Simple One Phase Model of a Geothermal Field," New Zealand Journal of Science, Vol.20,19,(1977).

4 Sorey,M.L..Fradkin,L.J.:"Validation and Comparison of Different Models of the Wairakei Geothermal Reservoir," Fifth Workshop on Geothermal Reservoir Engineering, Stanford University, (Dec. 12-14, 1979), SGP-TR-40.

5 Fradkin;L.J.:"Identification of the Wairakei Geothermal System," Water Resoursers Research, Vol.17. 921, (Aug. 1981).

6 Fradkin,L.J.,Sorey,M.L.,McNabb,A.:"On Identification and Validation of Some Geothermal Models," Water Resources Research, Vol.17, 929, (Aug. 1981).

7 Brigham, W.E.,Neri,G.:"A Depletion Model for the Gabbro Zone (Northern Part of Larderello Field)," Proceedings Second DOE-ENEL Workshop for Cooperative Research in Geothermal Energy, (Oct.20-22, 1980), LBL-11555.

8 Grant,M.A.:"Geothermal Reservoir Modeling," Geothermics, Vol.12, No.4, 251, (1983).

9 Castanier,L.M.,Brigham,W.E.:"Use of Lumped Parameter Modeling for Geothermal Engineering," SPE Cal. Regional Meeting, Ventura, CA, March 23-25, 1983, SPE 11730.

10 Castanier,L.M.,Sanyal,S.K.,Brigham,W.E. :"A Practical Analytical Model for Geothermal Reservoir Simulation," SPE Cal. Regional Meeting, Los Angeles, Ca, April 9-11, 1980, SPE 8887.

11 Miller,F.G.:"Theory of Unsteady-State Influx of Water in Linear Reservoirs," J.Inst.Pet., Vol.48, 365,(Nov. 1962).

12 Nabor,G.W.,Barham,R.H.:"Linear Aquifer Behavior," Jour.Pet.Tech., Trans. AIME Vol.231, 561, (May 1964).

13 Hurst, W.:"The Simplification of the Material Balance Formulas by the Laplace Transformation," Trans. AlME Vol.213, 292. (1958).

14 Carter,R.D.,Tracy,G.W.:"An Improved Method for Calculating Water Influx,"J.Pet.Tech.(Dec.1960) 38. 
15 Chatas,A.T.:"A Practical Treatment of Nonsteady-state Flow Problems in Resevoir Systems," From Pet.Engin. Series Begin. May, 1953, PET.E. 438-17.

16 Martin.J.C.:"Analysis of Internal Steam Drive in Geothermal Reservoirs," J.Pet.Tech. 1493 (Dec. 1975).

17. Macias Chapa,L.: Personal communication, 1984.

18 Morris,C.W.,Campbell,D.A.:"Geothermal Reservoir Energy Recovery: A Three Dimensional Simulation Study of the East Mesa Field," SPE 8229, Annual Fall Meeting of the SPE, 1979, Las Vegas, NV.

19 Schilthuis, R.J.:"Active Oil and Reservoir Energy", Trans. AIME (1936) 118,31.

20 Van Everdingen;A.F.,Hurst,W.:"The Application of the Laplace Transformation to Flow Problems in Reservoirs", Trans. AIME (1949) 186, 305.

21 Stehfest,H.:"Algorithm 368, Numerical Inversion of Laplace Transforms," D-5 Communications of the ACM (Jan.1970), 13, No.1, 47-49.

22 Fetkovitch, M.J.:"A Simplified Approach to Water Influx Calculations-Finite Aquifer Systems," Jour.Pet.Tech., AIME 814 (July 1971).

23 Regalado,J.R.:"A Study of the Responce to Exploitation of the Svartsengi Geothermal Field, SW-Iceland," UNU Geothermal Training Programme, Iceland, Report 1981-7.

24 Kjaran,S.P.,Eliasson,J.,Halldorsson,G.K.:"Svartsengi, Reservoir Engineering Study",National Energy Authority, Reykjavik, Iceland, OS 80021/ROD10JHD 17.(Report in Icelandic), 1980.

25 Kjaran,S.P.,Halldorsson,G.K.,Thorhallsson,S.,Eliasson,J.:"Reservoir Engineering Aspects of Svartsengi Geothermal Area," Geothermal Research Council, Trans. Vol.3,(Sep. 1979).

26 Kjaran,S.P.,Eliasson,J.:"Geothermal Reservoir Engineering Lecture Notes," UNU Geothermal Training Programme, Report 1983-2.

27 Barelli,A.,Palama,A.:"On Some Computationa Methods of Unit Responce Functions From Varying-Rate Data," Geothermics, Vol.9, pp 261-269, 1980.

28 Kjaran,S.P.,Halldorsson,G.K.,Eliasson,J.:"Svartsengi: Drawdown with Production,"Vatnaskil h/f Consulting Engineers, 1983, (in Icelandic).

29 Gudmundsson,J.S.:"Injection Testing in 1982 at the Svartsengi HighTemperature Field in Iceland," Transactions Geothermal Resource Council,7.Oct. 1983.

30 Thorhallsson,S.: Personal communication, 1983. 
31 Grant,M.A.,Sorey.M.L.:"The Compressibility and Hydraulic Diffusivity of a Water-Steam Flow," Water Resources Research, Vol. 15, No. 3, 684 (June 1979).

32 Atkinson,P.G.,Celati,R.,Corsi,R.,Kucuk.F., and Ramey,H.J.Jr.:"Thrmodynamic Behavior of the Bagnore Geothermal Field, Geothermics, Vol 7, No.2-4, 185208. 


\section{DEFINITIONS}

Dimensionless pressure:

Radial geometry: Linear geometry:

$p_{D}=\frac{2 \pi k h \Delta p}{q \mu} \quad p_{D}=\frac{k b h \Delta p}{q \mu L}$

Dimensionless time:

Radial geometry: Linear geometry:

$t_{D}=\frac{k t}{\varphi \mu c r^{2}} \quad t_{D}=\frac{k t}{\varphi \mu c L^{2}}, \quad(L=1$ if $\infty)$

Dimensionless cumulative production:

Radial geometry: Linear geometry:

$Q_{D}=\frac{Q}{2 \pi h \varphi c r_{w}^{2} \Delta p} \cdot Q_{D}=\frac{Q}{b h L \varphi c \Delta p}$

Van Everdingen \& Hurst Influx Consant (pressure in Pa):

Radial geometry: Linear geometry:

$B=2 \pi \varphi c r_{w}^{2} h \frac{\theta}{360^{\circ}} \rho\left(\frac{k g}{P a}\right) \quad B=\varphi c b h L \rho\left(\frac{k g}{P a}\right) \quad(L=1$ if $\infty)$

Schilthuis Influx Constant (pressure in meters of water):

Radial geometry: Linear geometry:

$K=\frac{2 \pi k h \rho_{w g}^{2} g}{\mu \ln \frac{r_{e}}{r_{w}}} \frac{\theta}{360^{\circ}}\left(\frac{k g}{m \cdot s}\right) \quad K=\frac{k b h \rho_{w}^{2} g}{\mu L}\left(\frac{k g}{m \cdot s}\right)$ 
TABLE-2. Example of history matching using the Schilthis method.

\begin{tabular}{|c|c|c|c|c|c|c|c|c|}
\hline (step of depletion) & $\begin{array}{l}t_{n} \\
(s)\end{array}$ & $\begin{array}{l}\Delta t_{n} \\
(s)\end{array}$ & $\begin{array}{c}w_{n} \\
(\mathrm{~kg} / \mathrm{s})\end{array}$ & $\begin{array}{l}\Delta h_{n} \\
(\mathrm{~m})\end{array}$ & $\begin{array}{l}W_{p n} \\
(\mathrm{~kg})\end{array}$ & $\begin{array}{l}\sum_{j=1}^{n} \Delta h_{j} \Delta t_{j} \\
(\mathrm{~m} \cdot \mathrm{s})\end{array}$ & \multicolumn{2}{|c|}{$\begin{array}{c}y_{n} \\
(\mathrm{~kg} / \mathrm{m} \cdot \mathrm{s})\end{array}$} \\
\hline 1 & $8.64 \times 10^{6}$ & $8.64 \times 10^{8}$ & 40 & 0.85 & $0.346 \times 10^{9}$ & $7.34 \times 10^{6}$ & $11.6 \times 10^{-8}$ & 47.14 \\
\hline 2 & $17.28 \times 10^{6}$ & $8.64 \times 10^{8}$ & 30 & 1.5 & $0.605 \times 10^{\theta}$ & $20.3 \times 10^{8}$ & $7.39 \times 10^{-8}$ & 29.80 \\
\hline 3 & $34.56 \times 10^{8} \mid$ & $17.28 \times 10^{6}$ & 60 & 4.0 & $1.642 \times 10^{9}$ & $89.4 \times 10^{6}$ & $4.47 \times 10^{-8}$ & 18.37 \\
\hline 4 & $60.48 \times 10^{8}$ & $25.92 \times 10^{8}$ & 120 & 11.5 & $4.752 \times 10^{9}$ & $387.5 \times 10^{6}$ & $2.97 \times 10^{-8}$ & 12.26 \\
\hline 5 & $86.41 \times 10^{6}$ & & 100 & 17.5 & $7.344 \times 10^{0}$ & $841.1 \times 10^{8}$ & $2.08 \times 10^{-8}$ & 8.73 \\
\hline
\end{tabular}




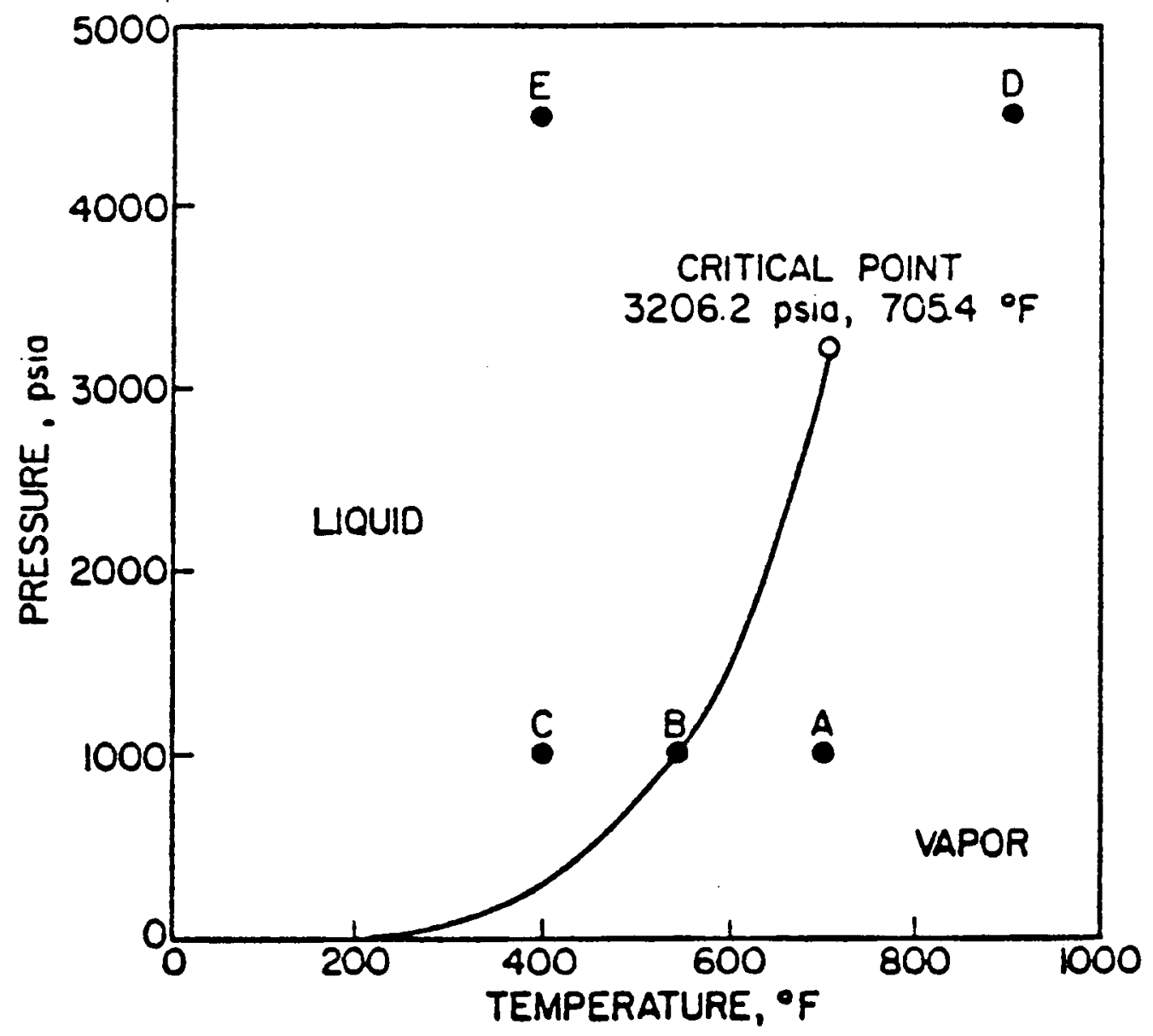

FIGURE 1. Pressure-temperature for pure water. (Whiting and Ramey, 1969) 


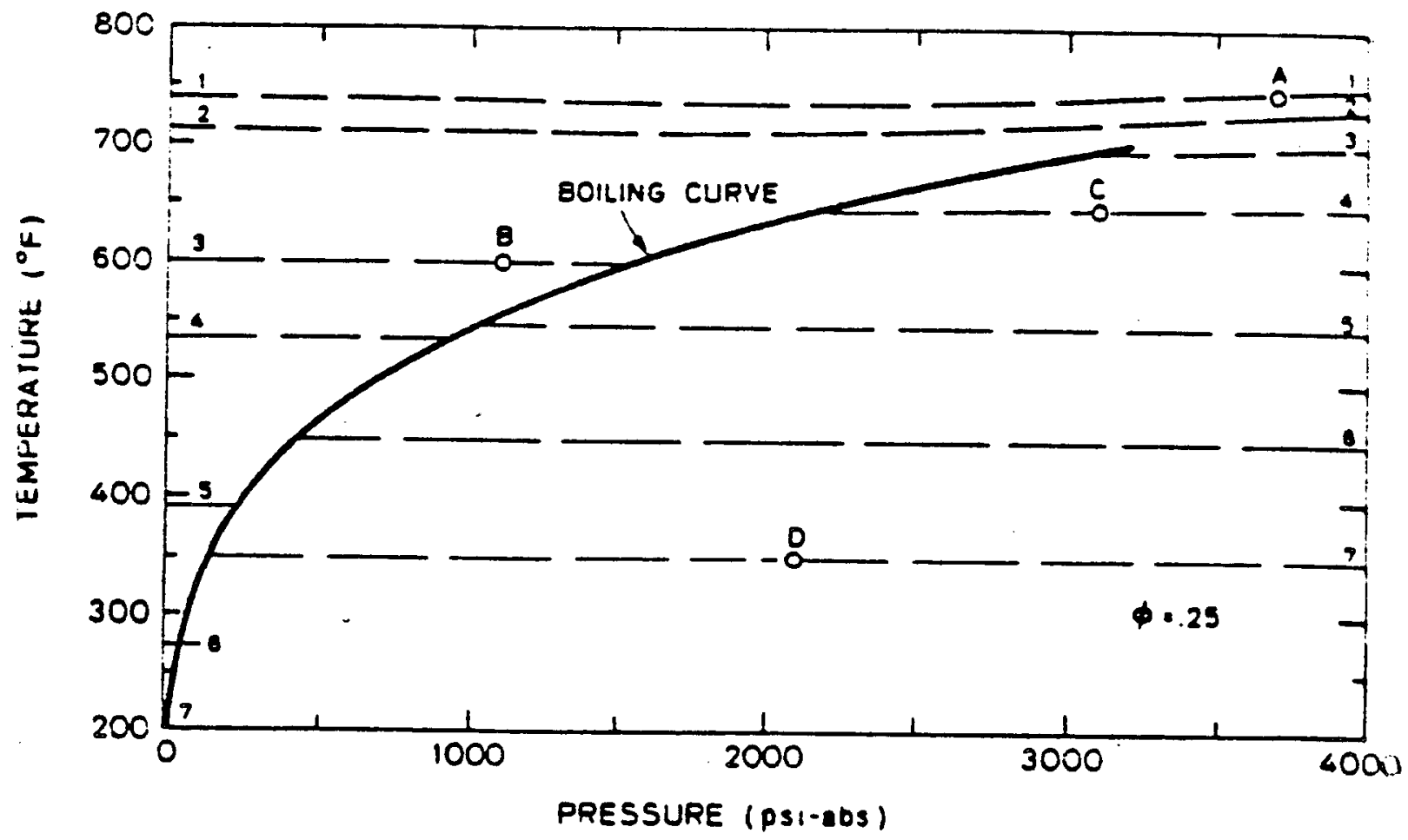

Pressure-temperature for geothermal reservoirs containing pure water an FIGURE 2. porosity of $25 \%$. (Martin, 1975) 


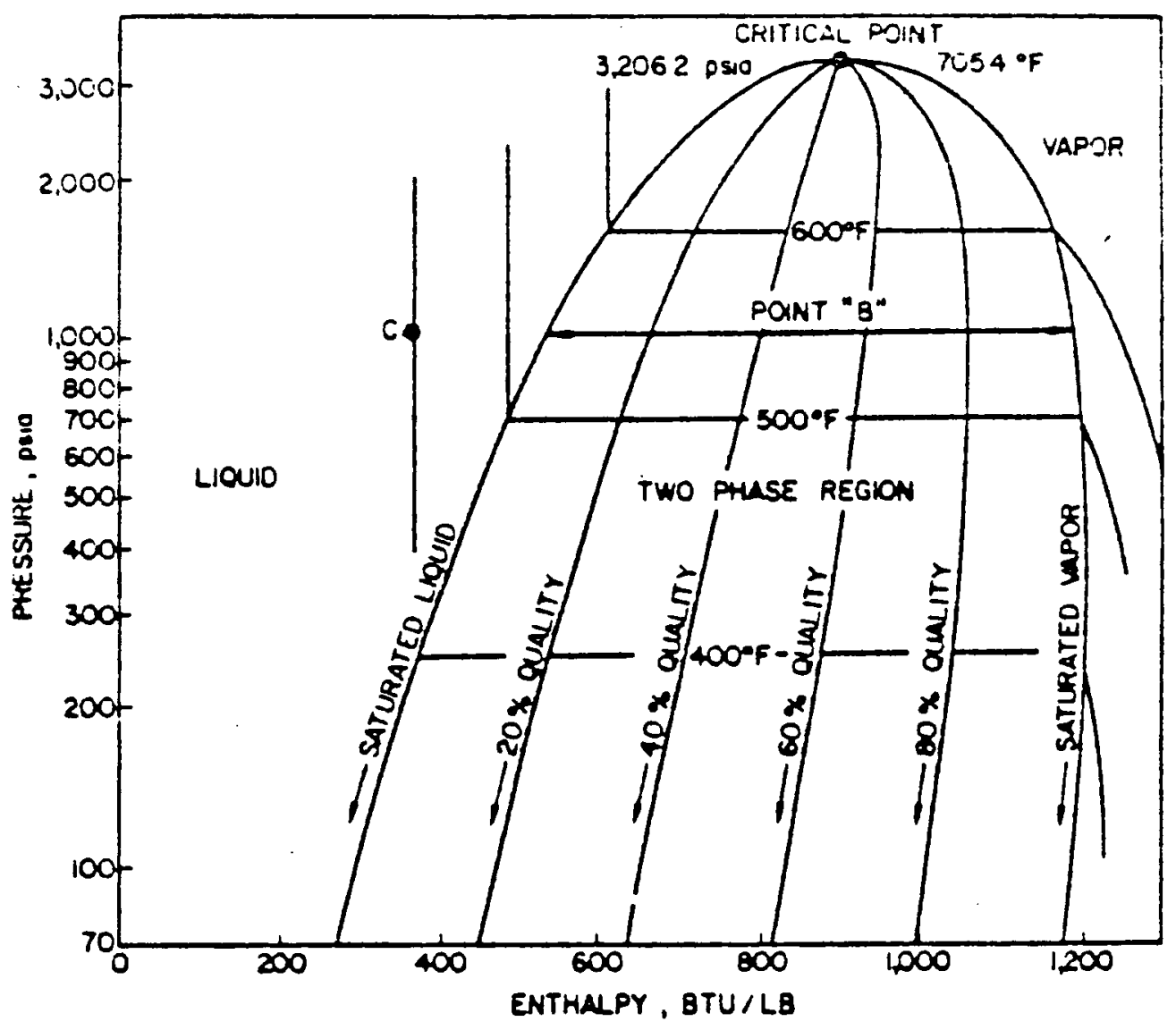

FIGURE 3. Pressure-enthalpy diagram for pure water. (Whiting and Ramey, 1969) 


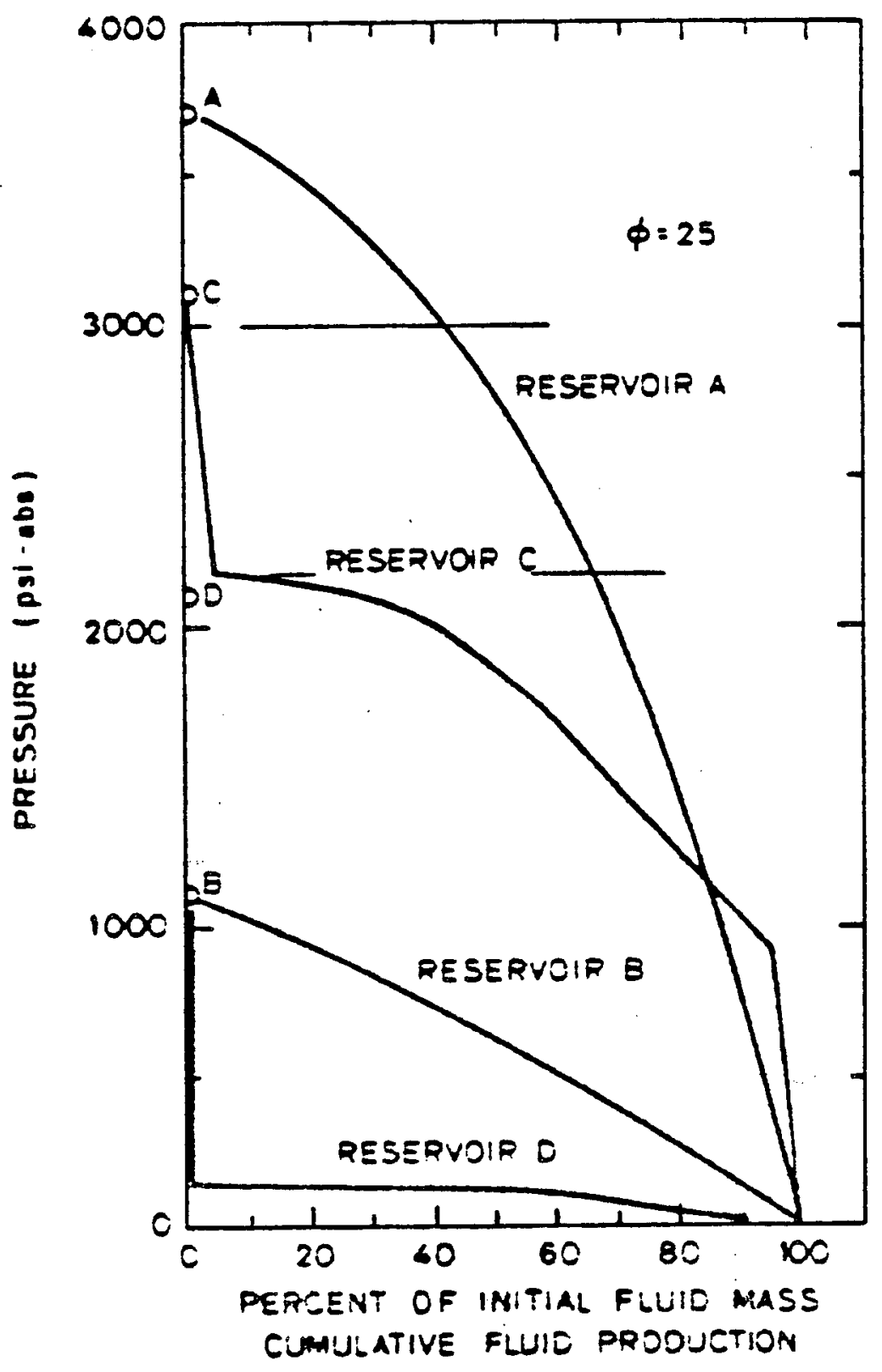

FIGURE 4. Pressure vs. cumulative fluid production for geothermal reservoirs contain pure water and a porosity of $25 \%$. (Martin, 1975) 


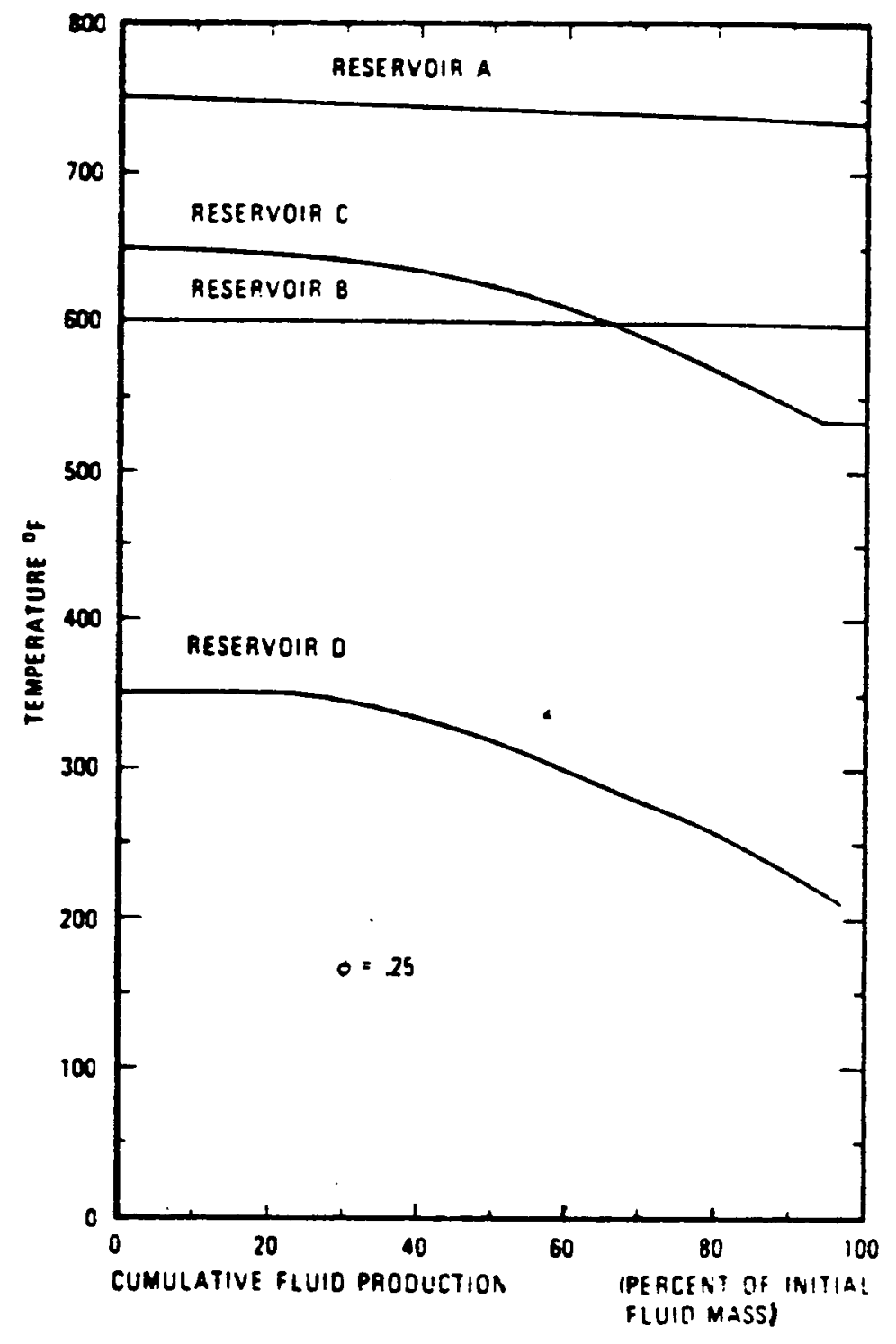

FIGURE 5. Temperature vs. cumulative fluid production for geothermal reservoirs containing pure water and porosity of 25\%. (Martin, 1975) 


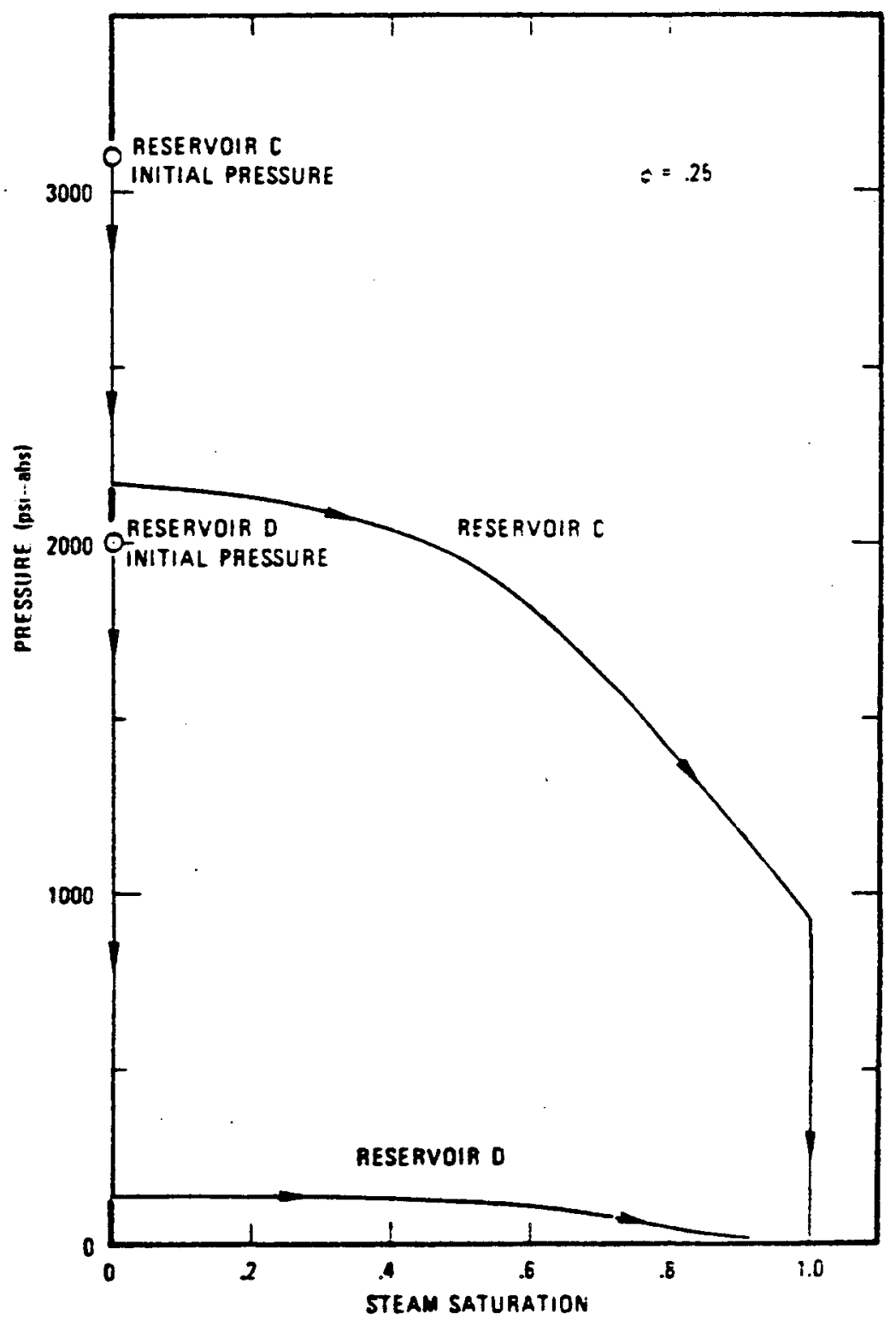

FIGURE 6. Pressure vs. steam saturation for geothermal reservoirs initially in compressed liquid state and porosity of 25\%. (Martin, 1975) 


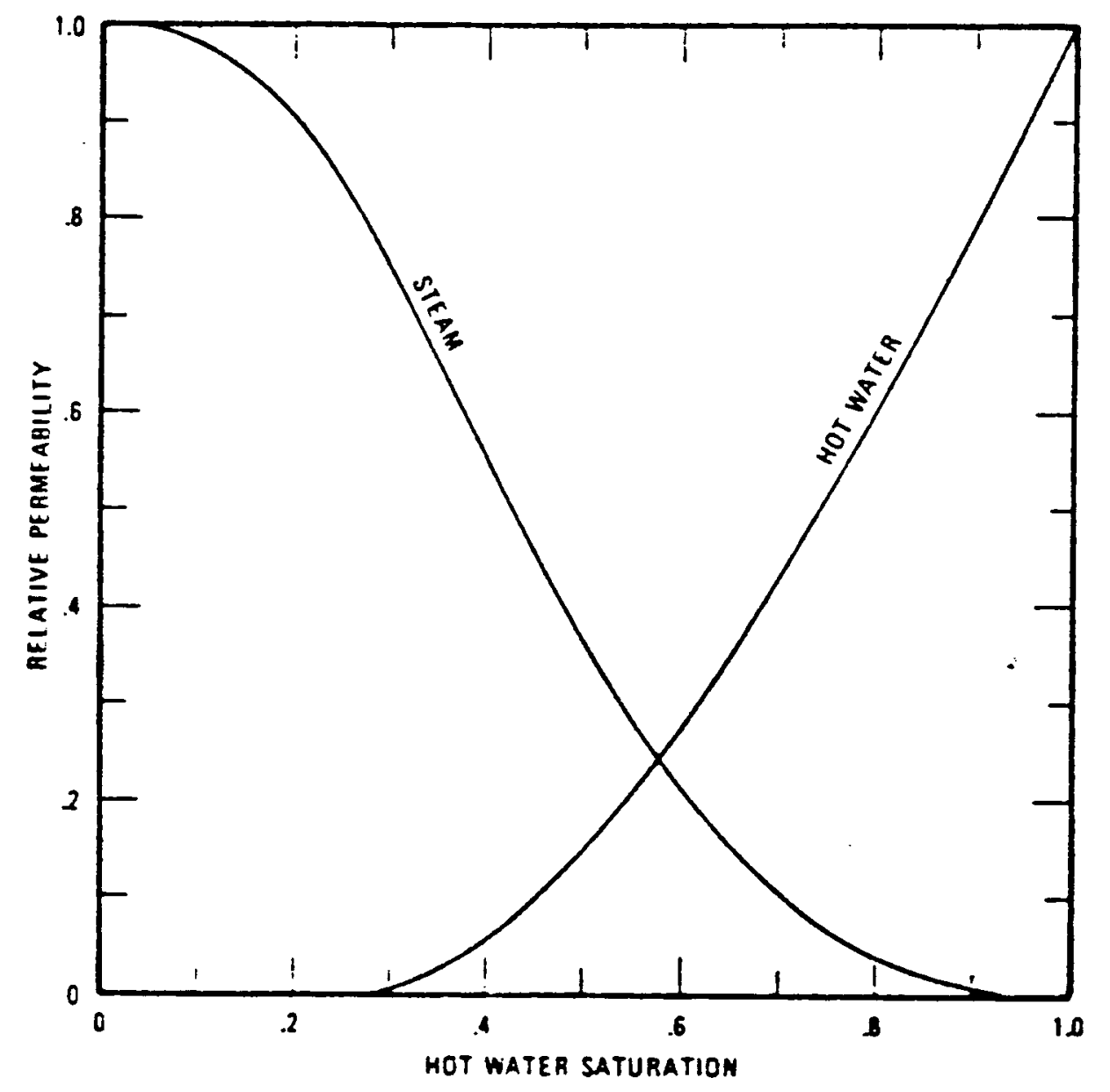

FIGURE 7. Steam and hot-water relative permeability curves. (Martin, 1975) 


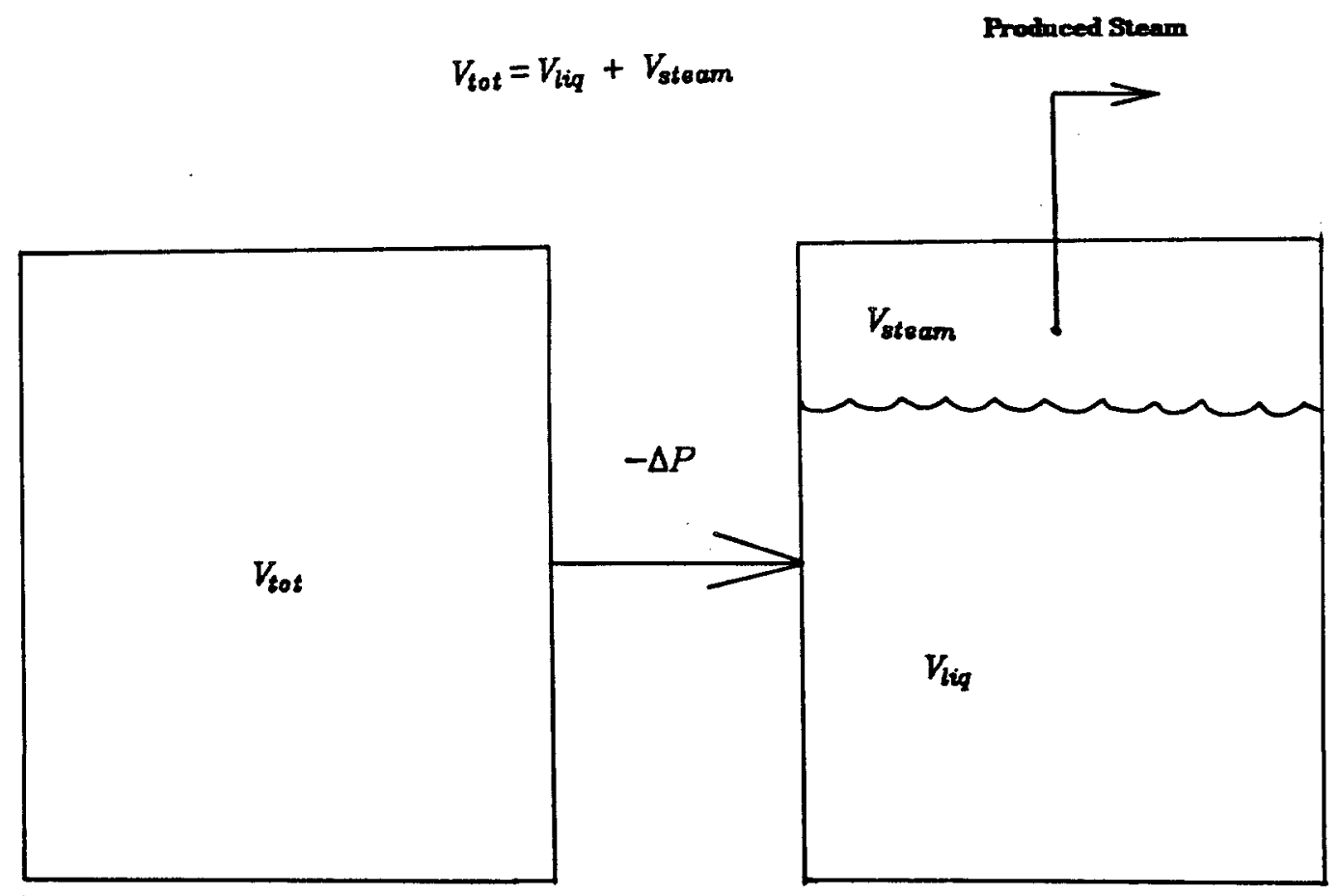

FIGURE 8.

Lumped-parameter model. (Macias-Chapa) 


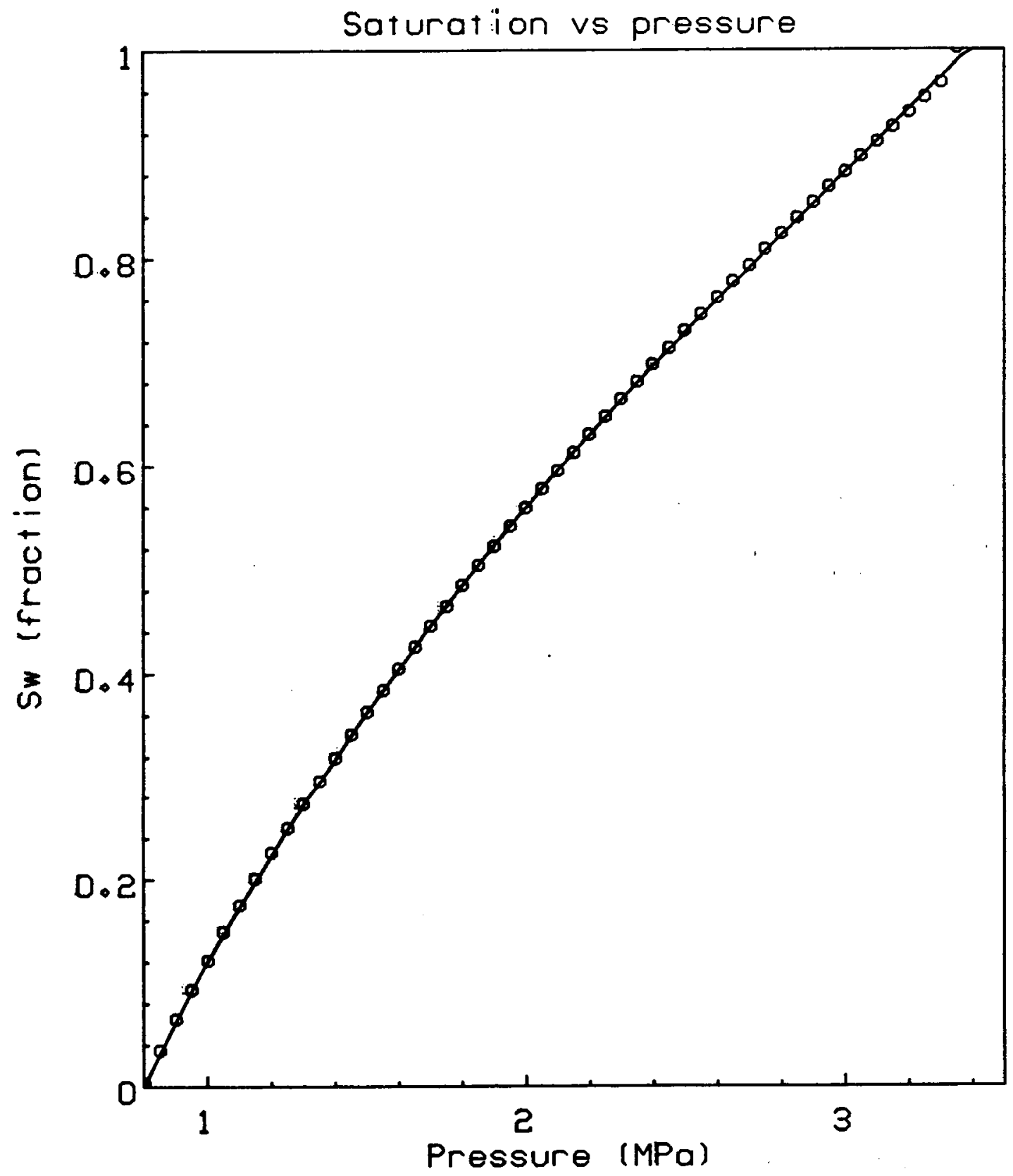

FIGURE 9. Pressure vs. liquid saturation for a geothermal reservoir of initial temperature $240^{\circ} \mathrm{C}$, and porosity $10 \%$. (Macias-Chapa) 


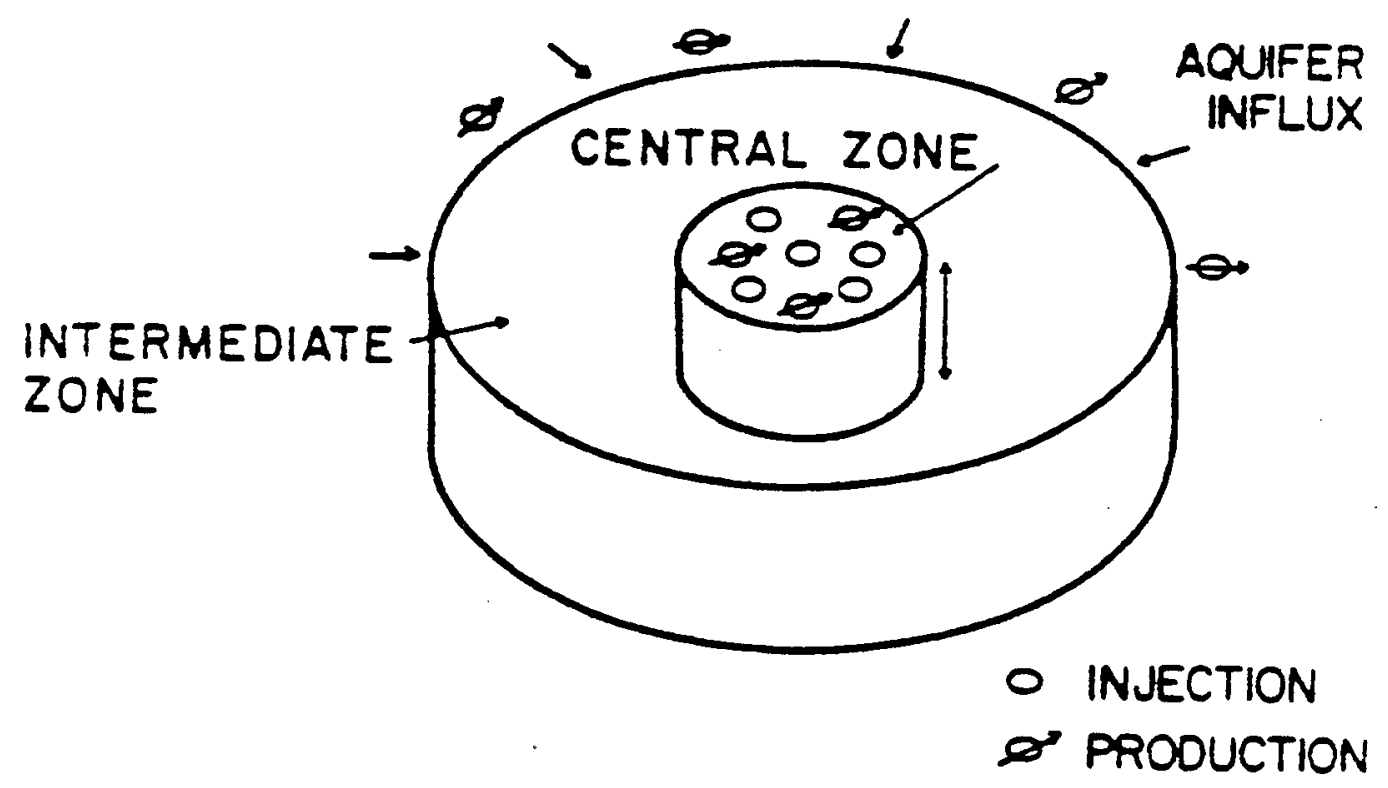

FIGURE-10. Reservoir model with three lumps. (Castanier, et al., 1980) 


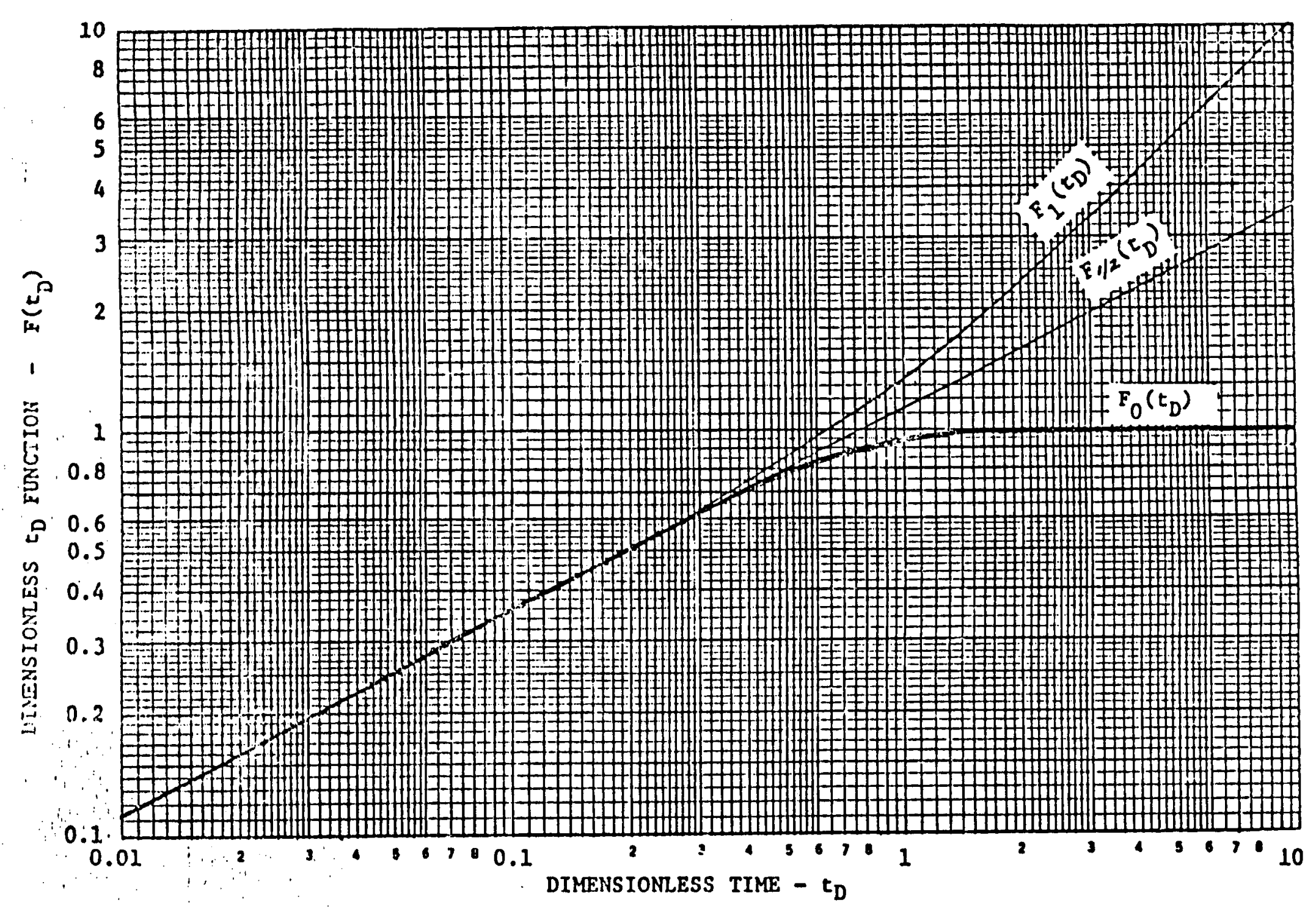

FIGURE-11. Dimensionless pressure and efflux functions for linear aquifers. (Nabor and Barham, 1964) 


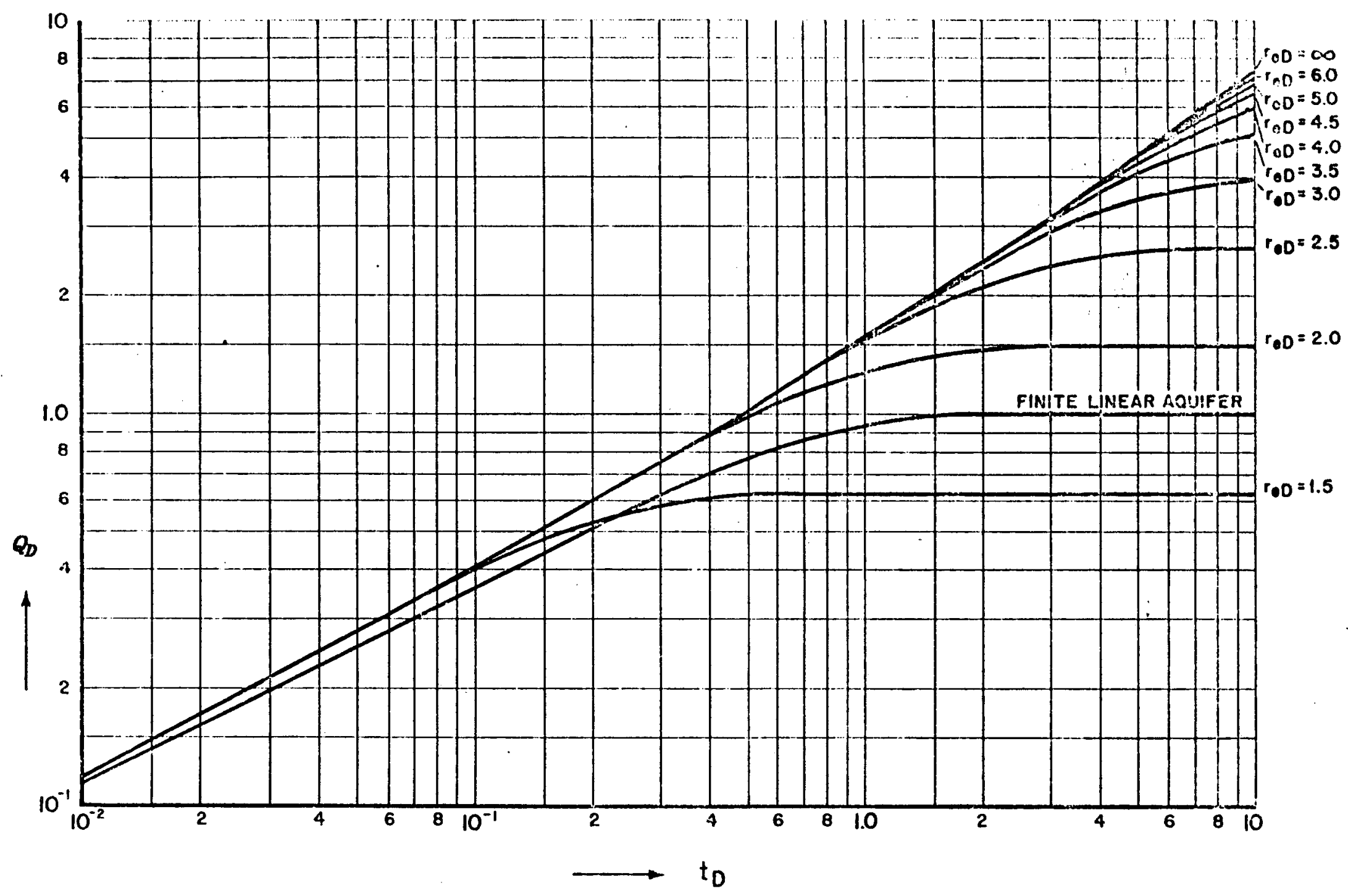

FIGURE-12a.Dimensionless water influx, constant terminal pressure, radial flow. (Van Everdingen and Hurst, 1949) 


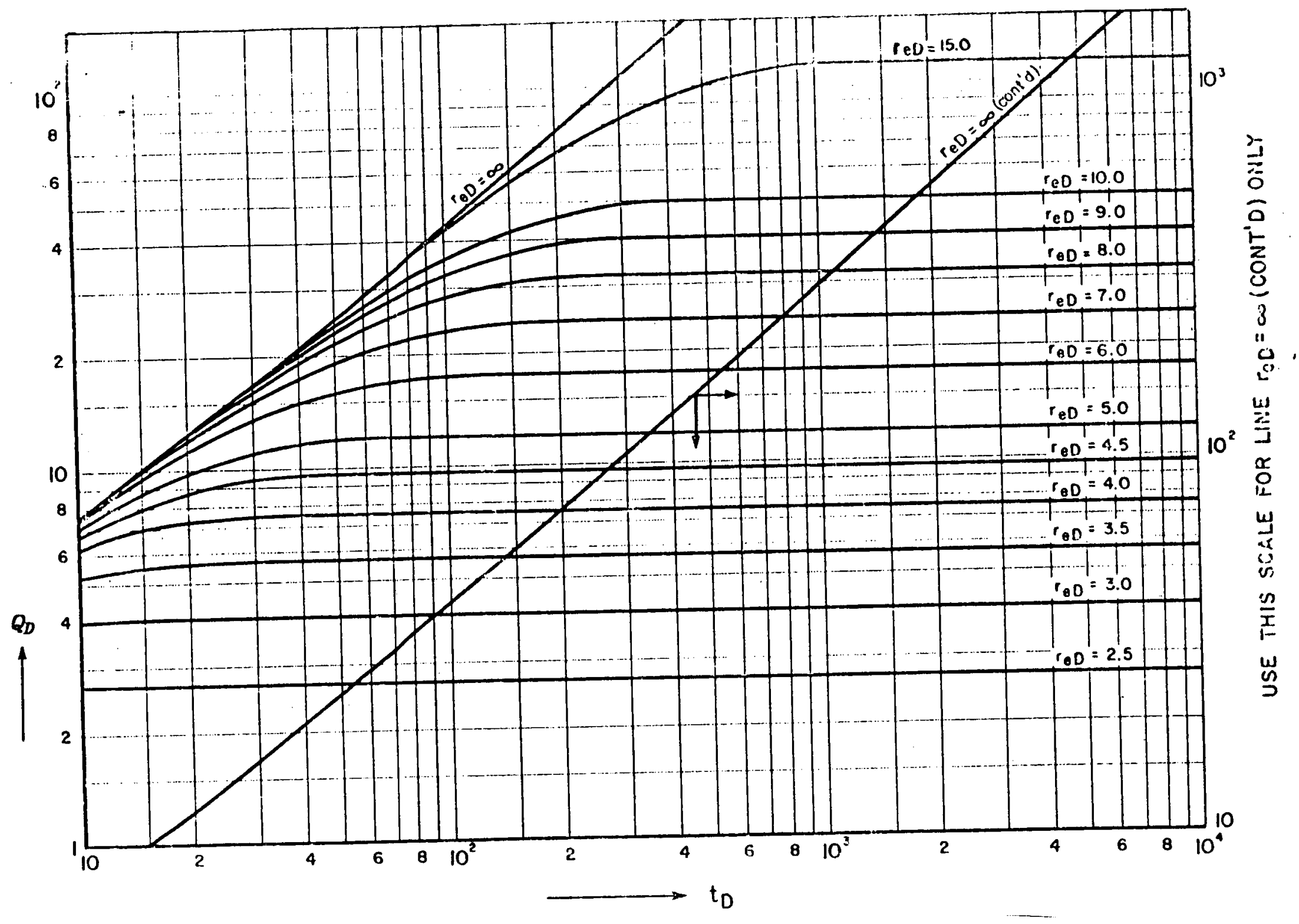

FIGURE-12b.Dimensionless water influx, constant terminal pressure, radial flow. (Van Everdingen and Hurst, 1949) 


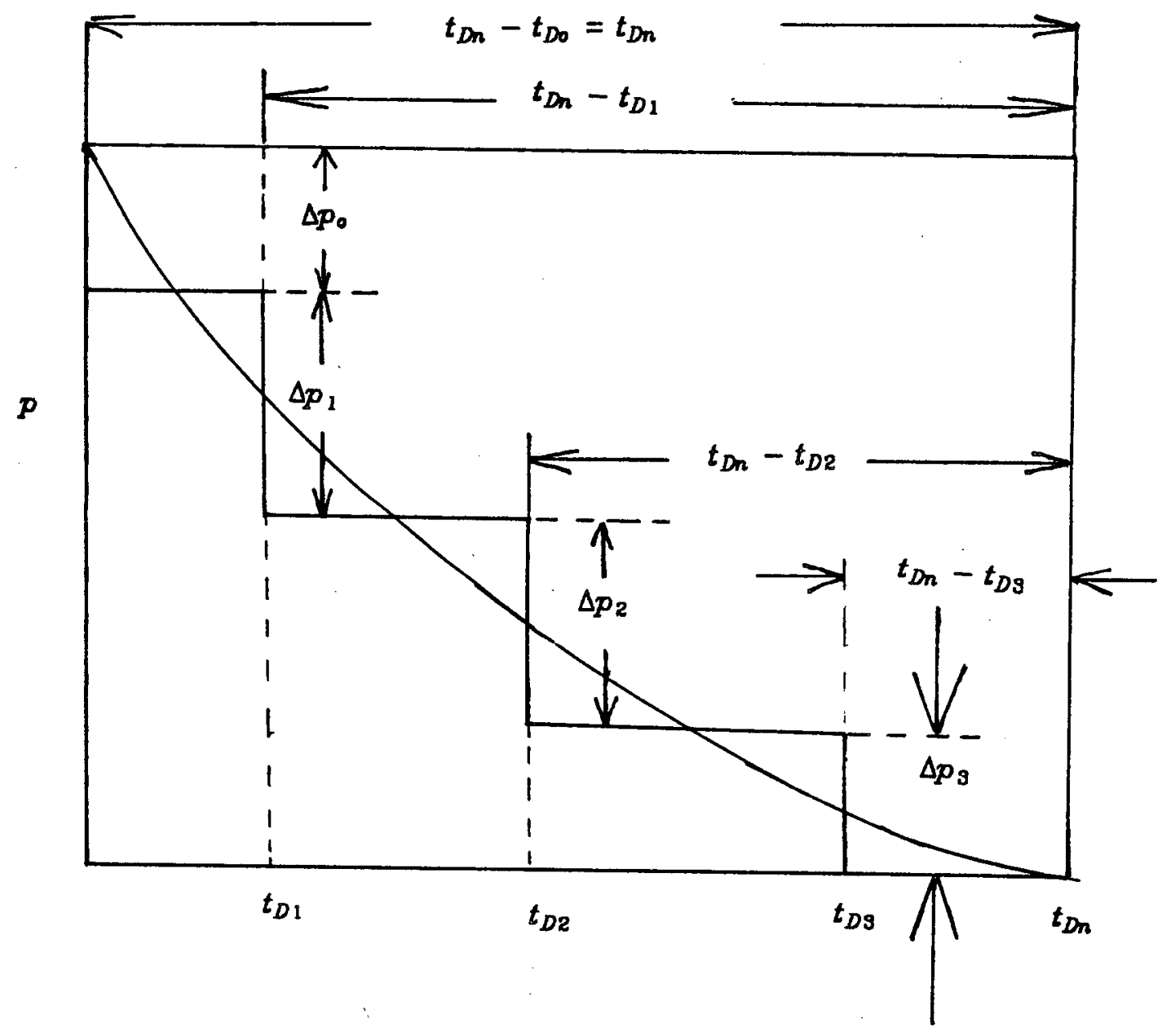

FIGURE-13. Dividing continuous pressure changes into constant pressure steps for superposition. 


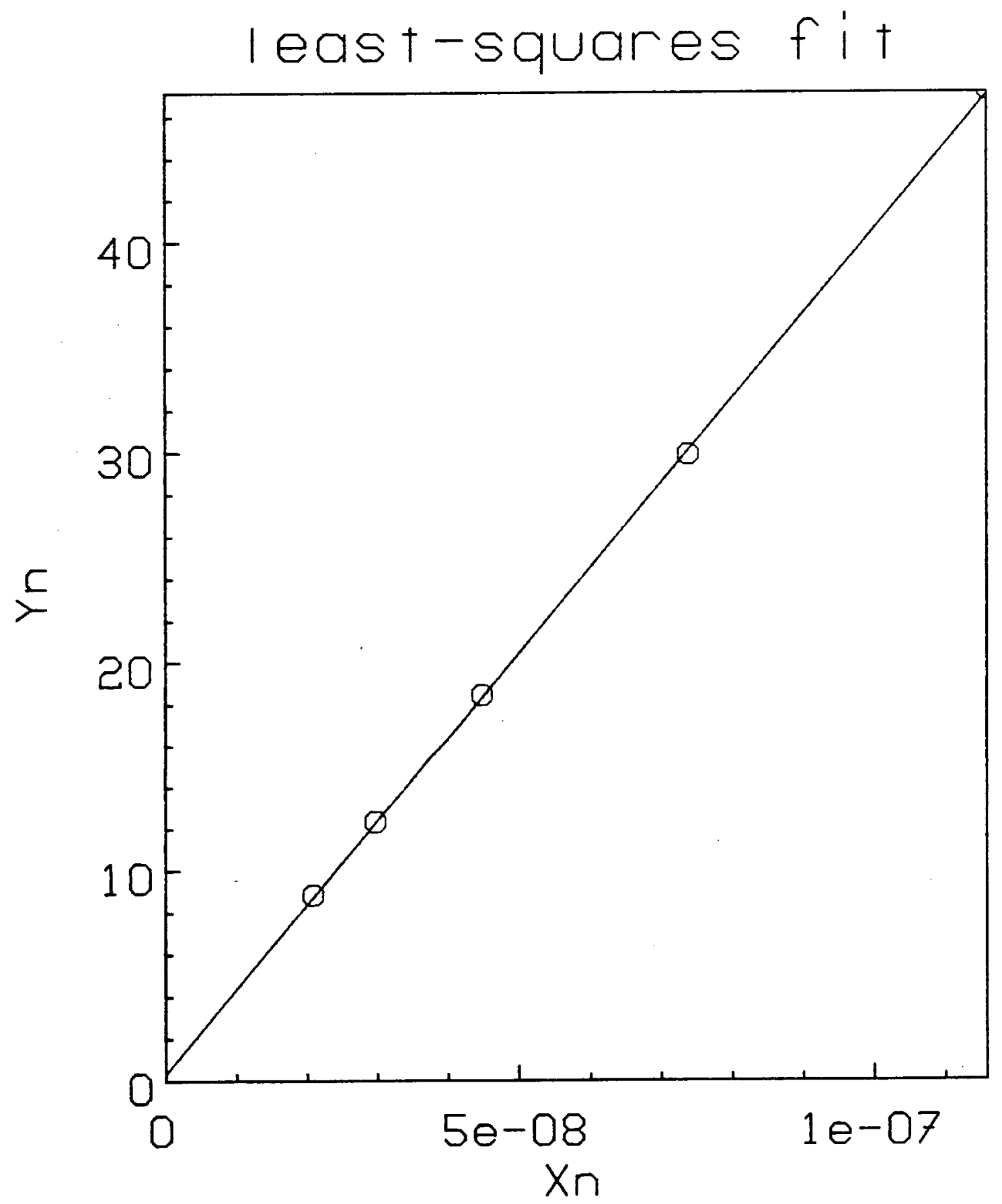

FIGURE-14. Least squares fit for the example given in history matching. 
The total mass rate at Svartsengi

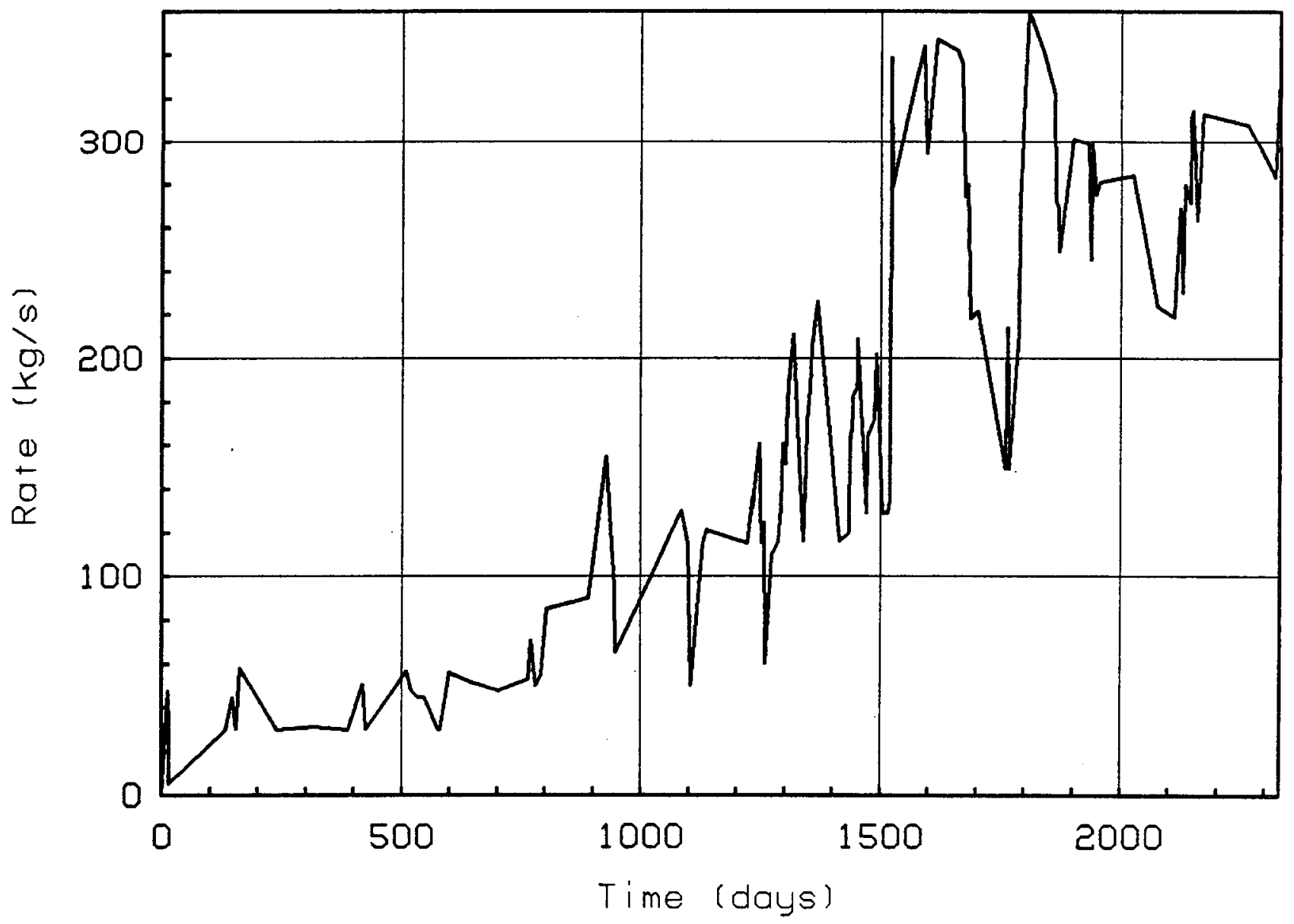

FIGURE-15a.Total mass rate produced at Svartsengi vs time. 


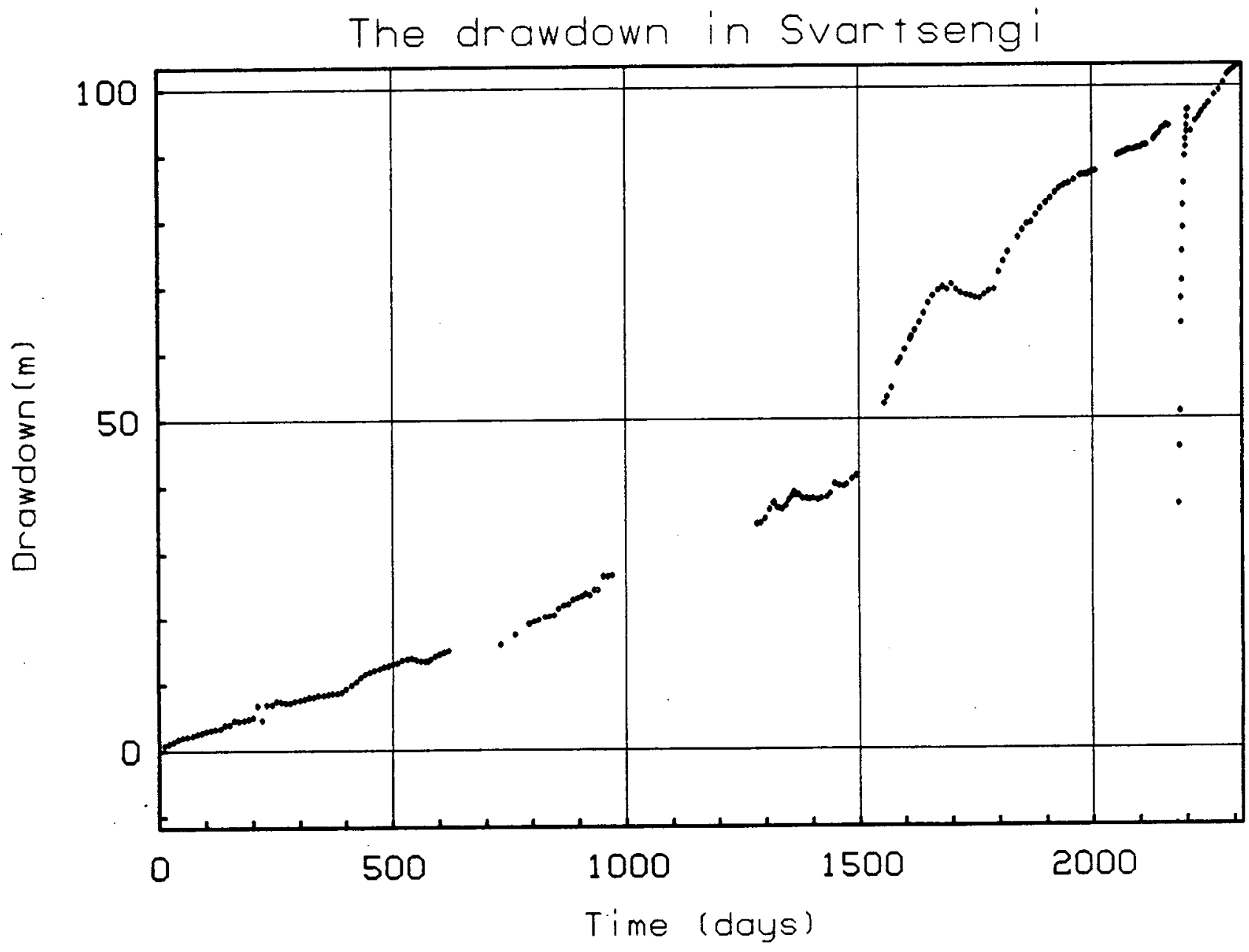

FIGURE-15b.Measured drawdown at Svartsengi vs. time. 


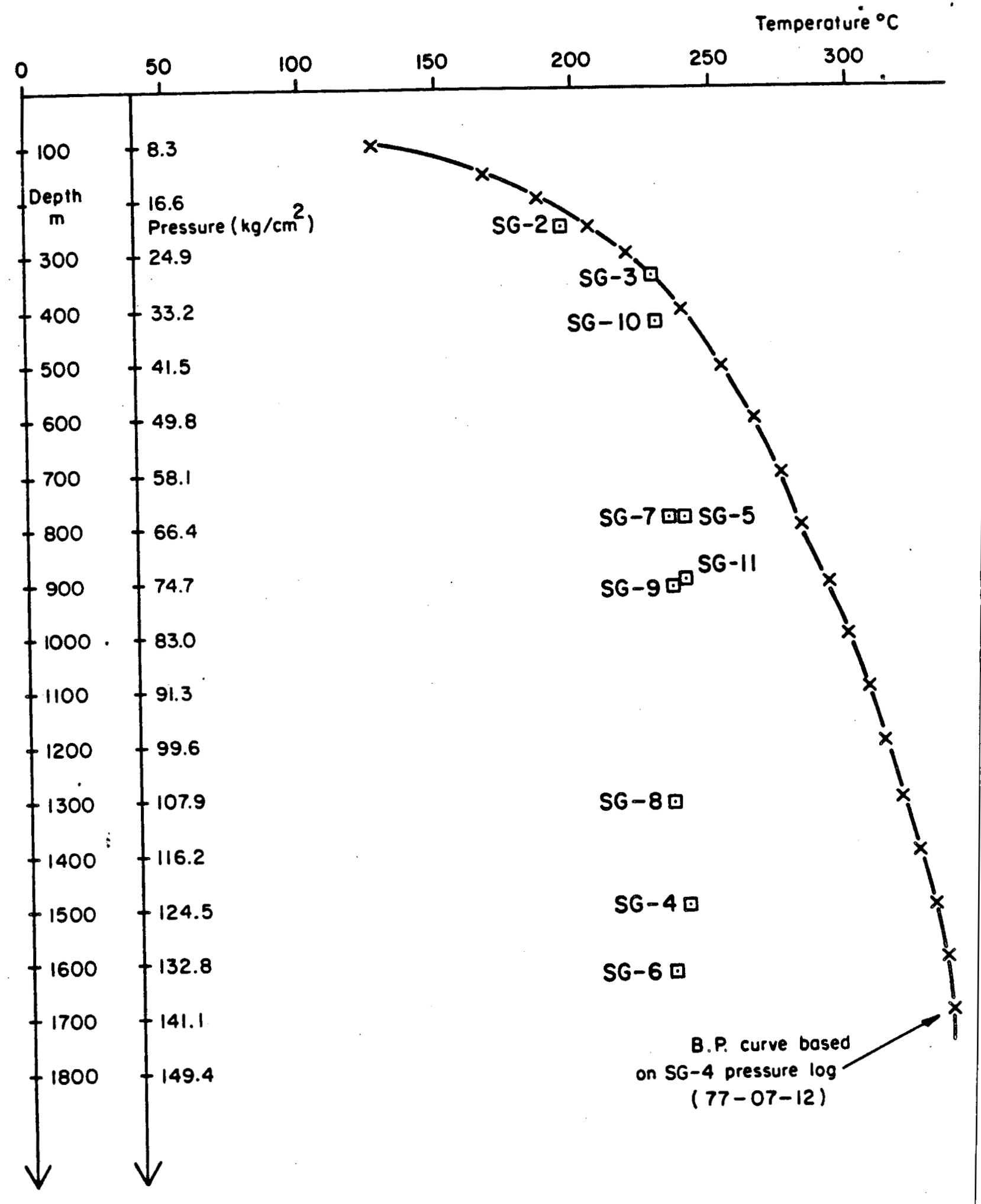

FIGURE-16. Pressure-temperature with depth at Svartsengi. 


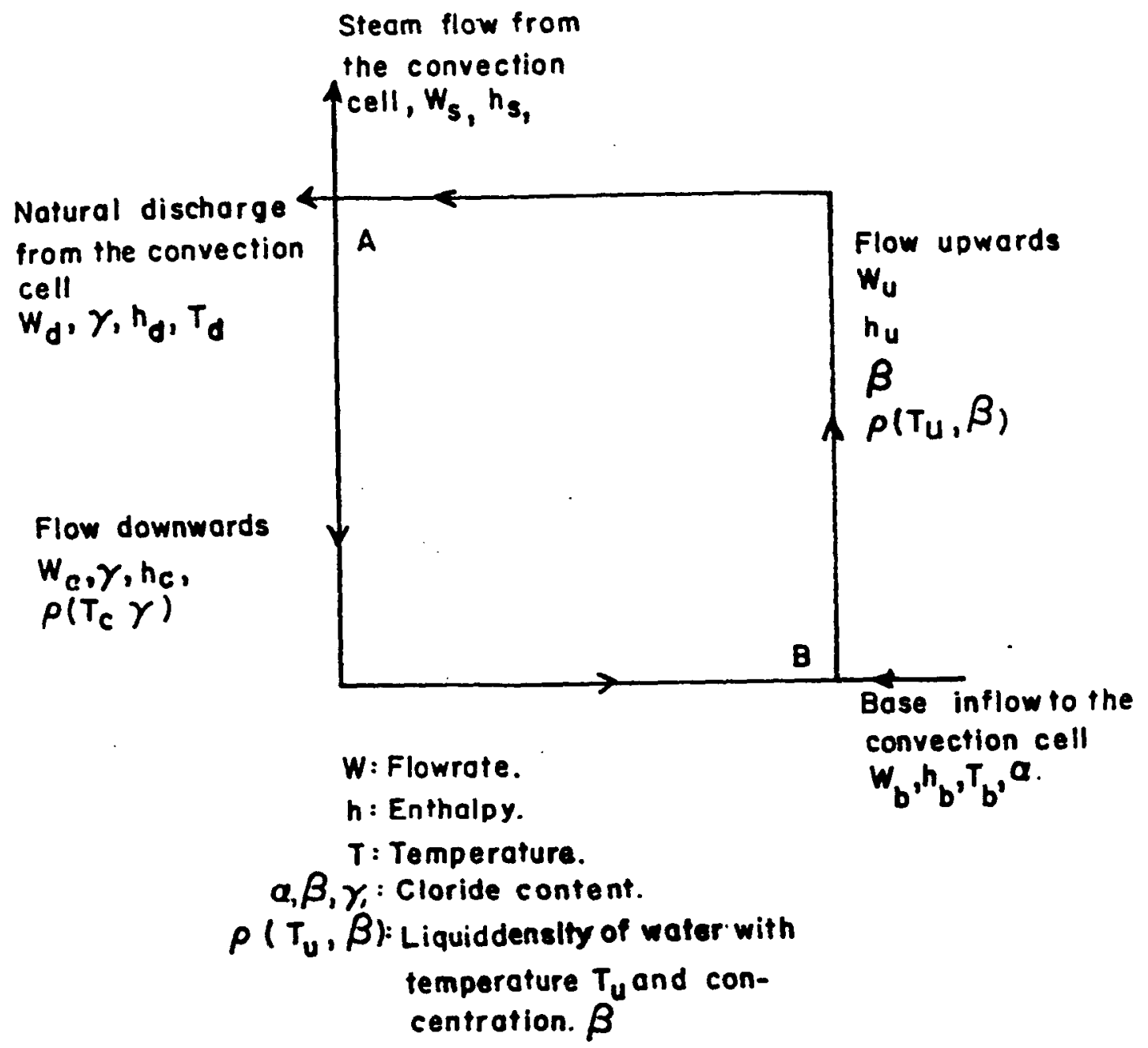

FIGURE-17. The convection model for Svartsengi. (Kjaran et al. 1980) 


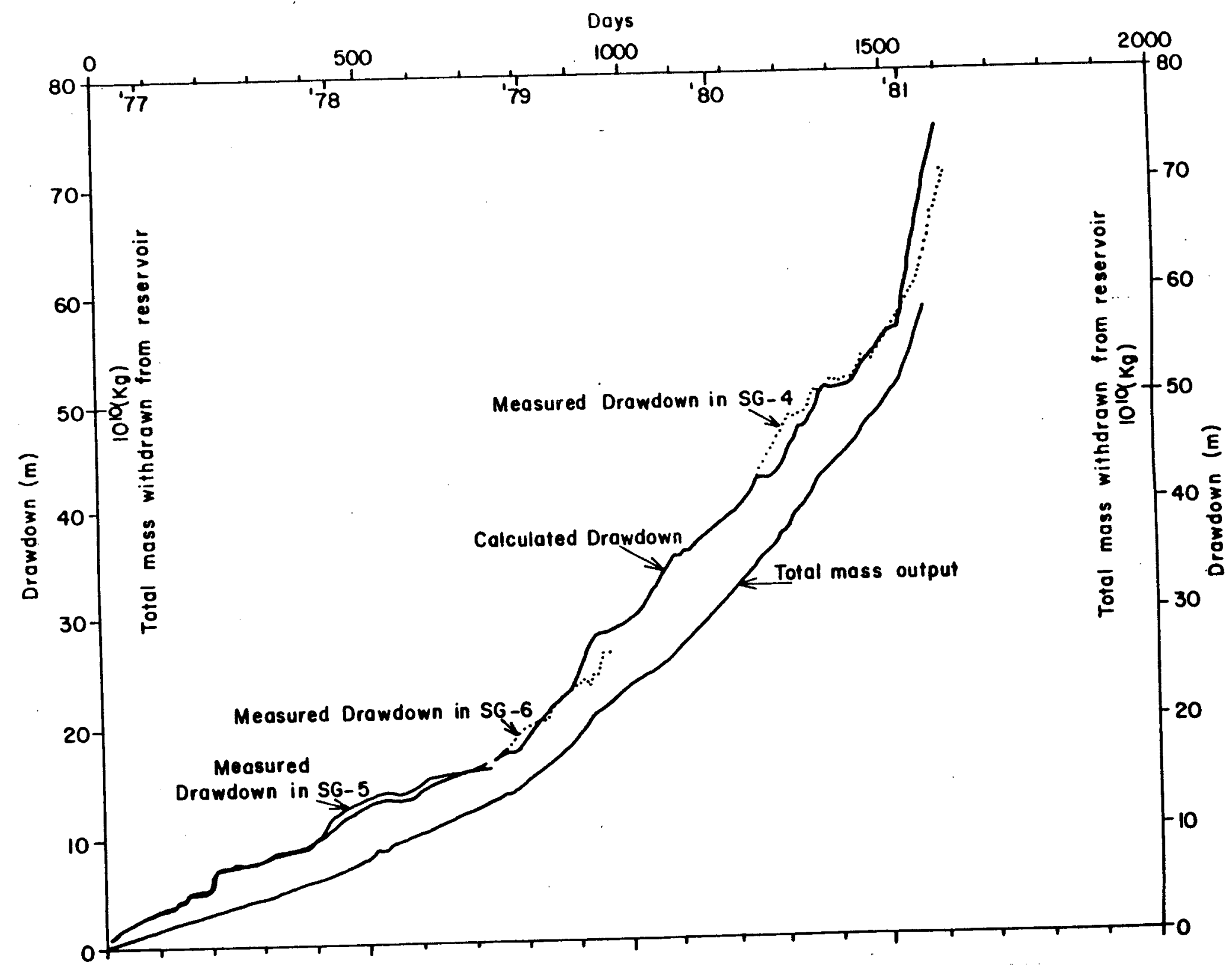

FIGURE-18. Measured and calculated drawdown using the hydrological model. 
86

68.

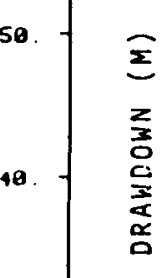

30 .

20 .

10

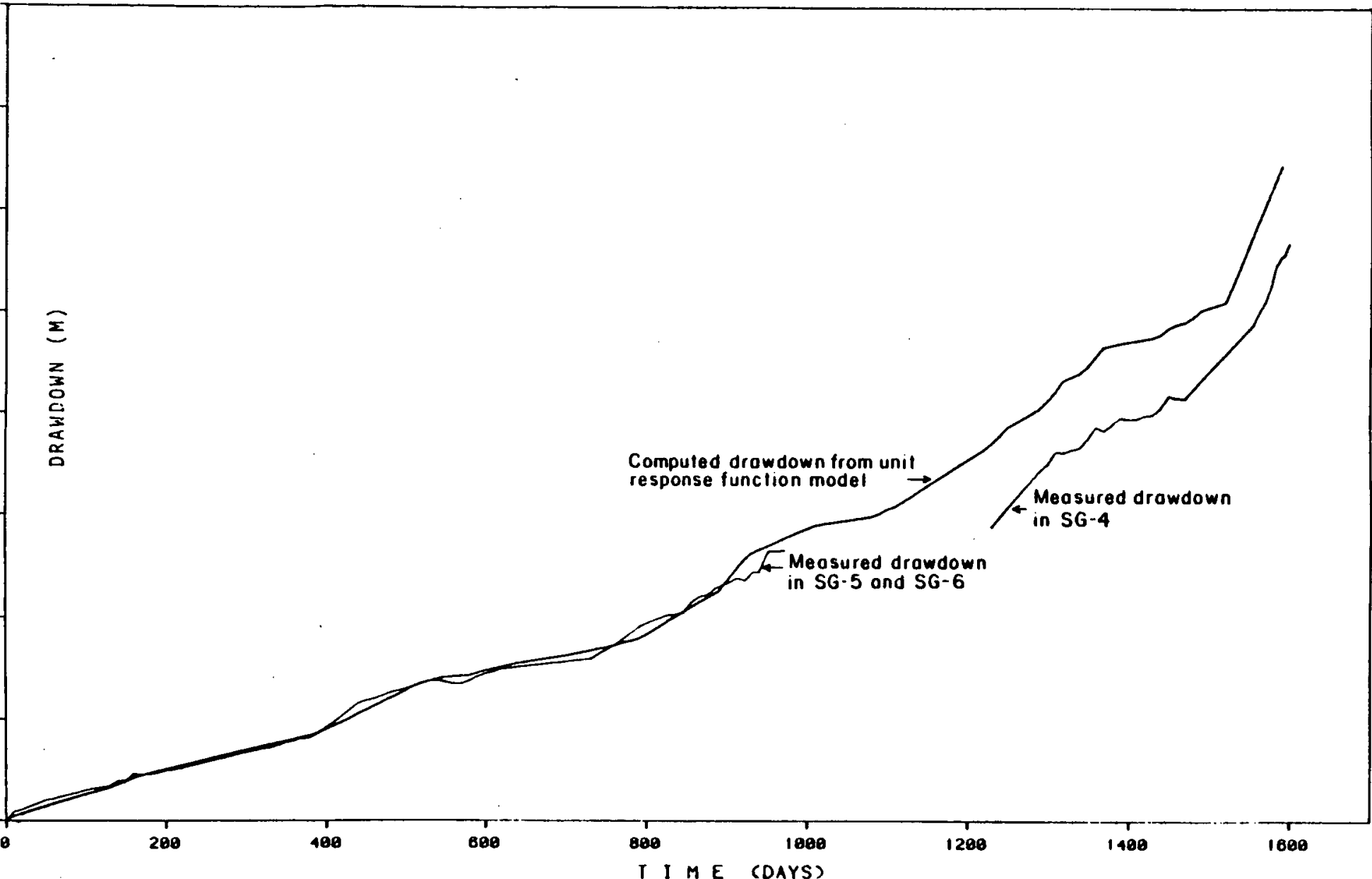

FIGURE-19. Measured and calculated drawdown using the unil response function model. (Regalado, 1981) 


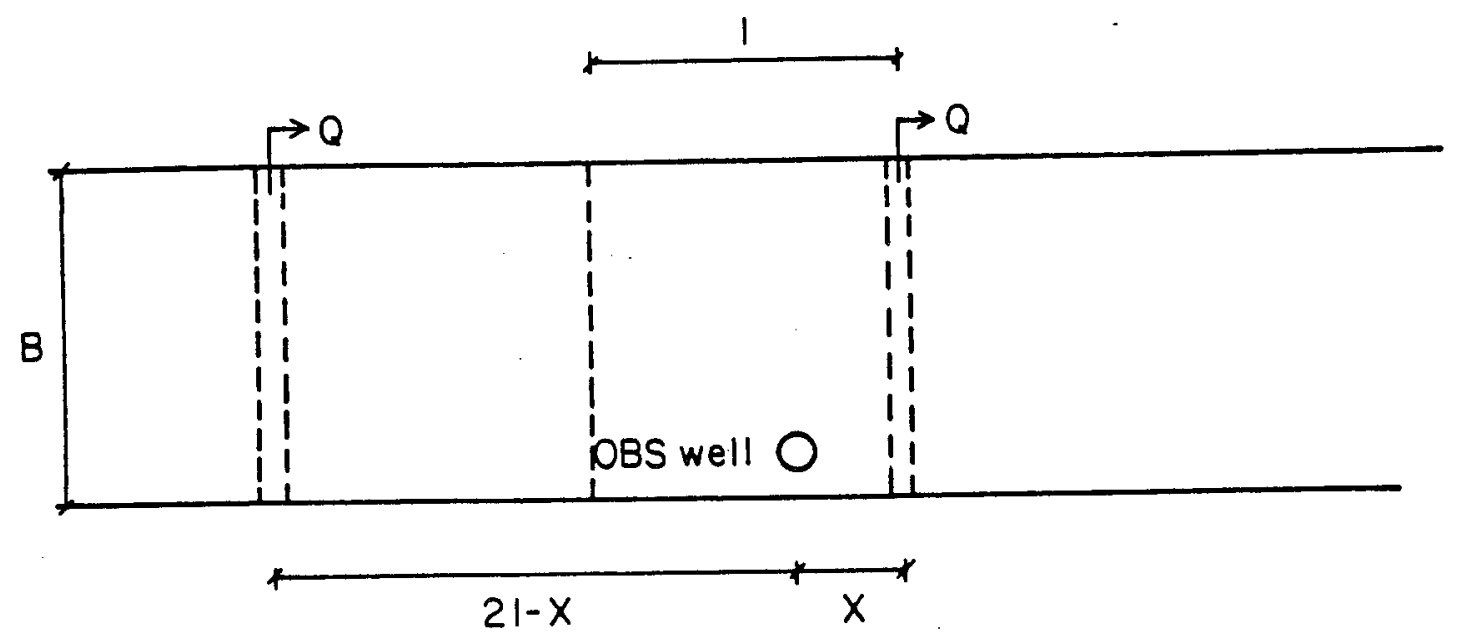

FIGURE-20. The esker model (Regalado, 1981) 


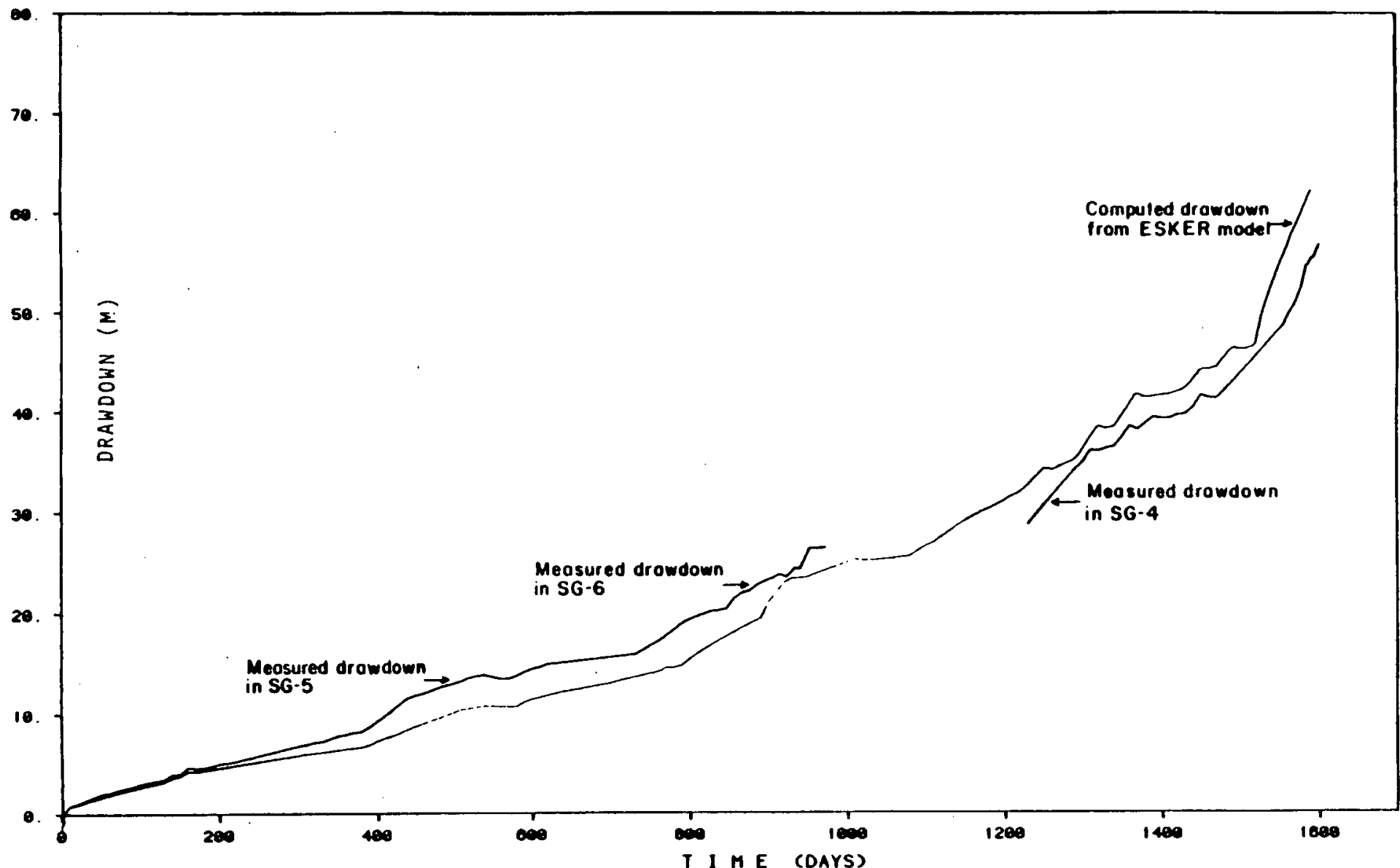

FIGURE-21. Measured and calculated drawdown using the esker model. (Regalado, 1981) 


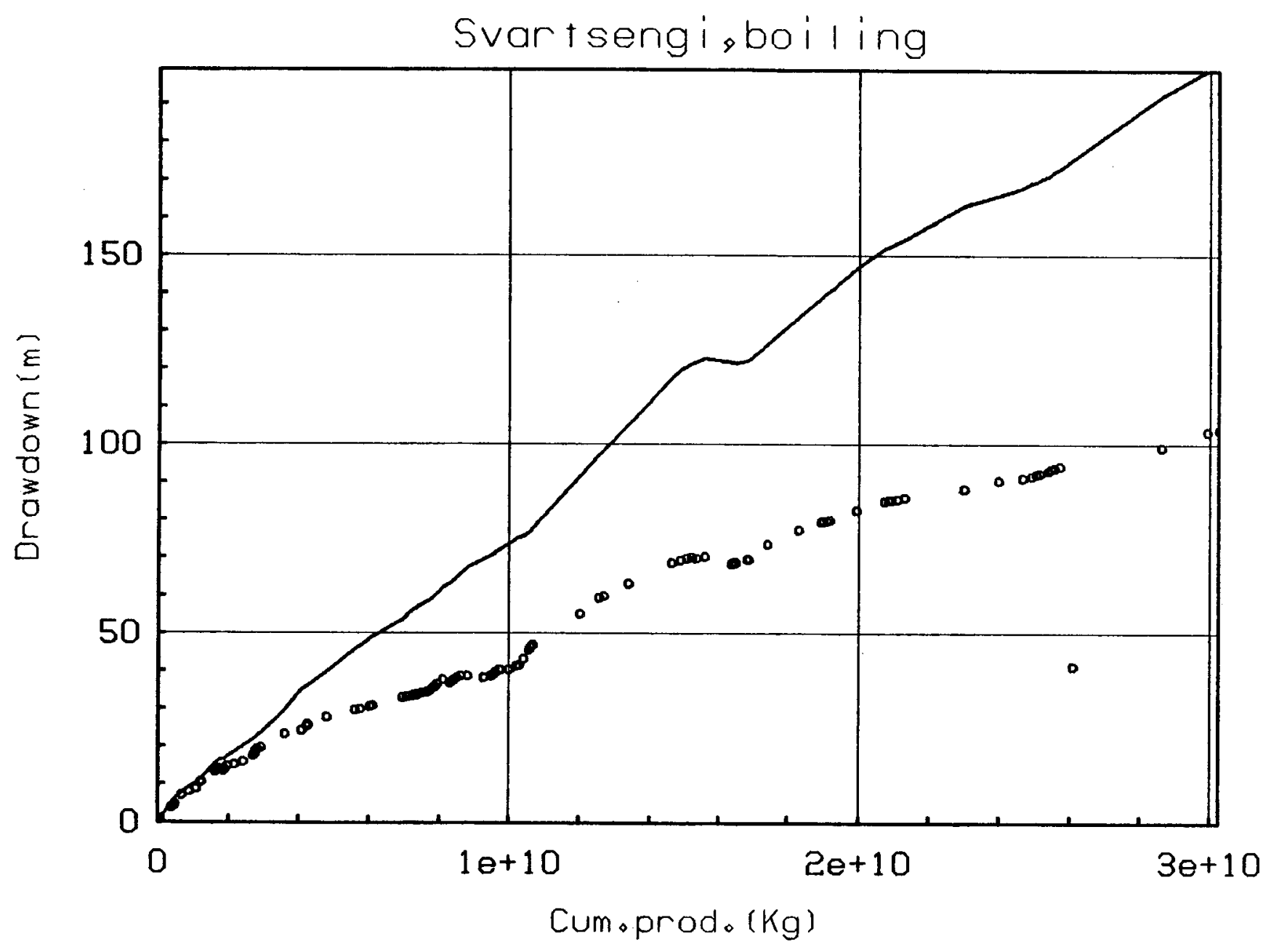

FIGURE-22. Measured and calculated drawdown using the boiling model with the constants reported by Thorhallsson. (1983) 


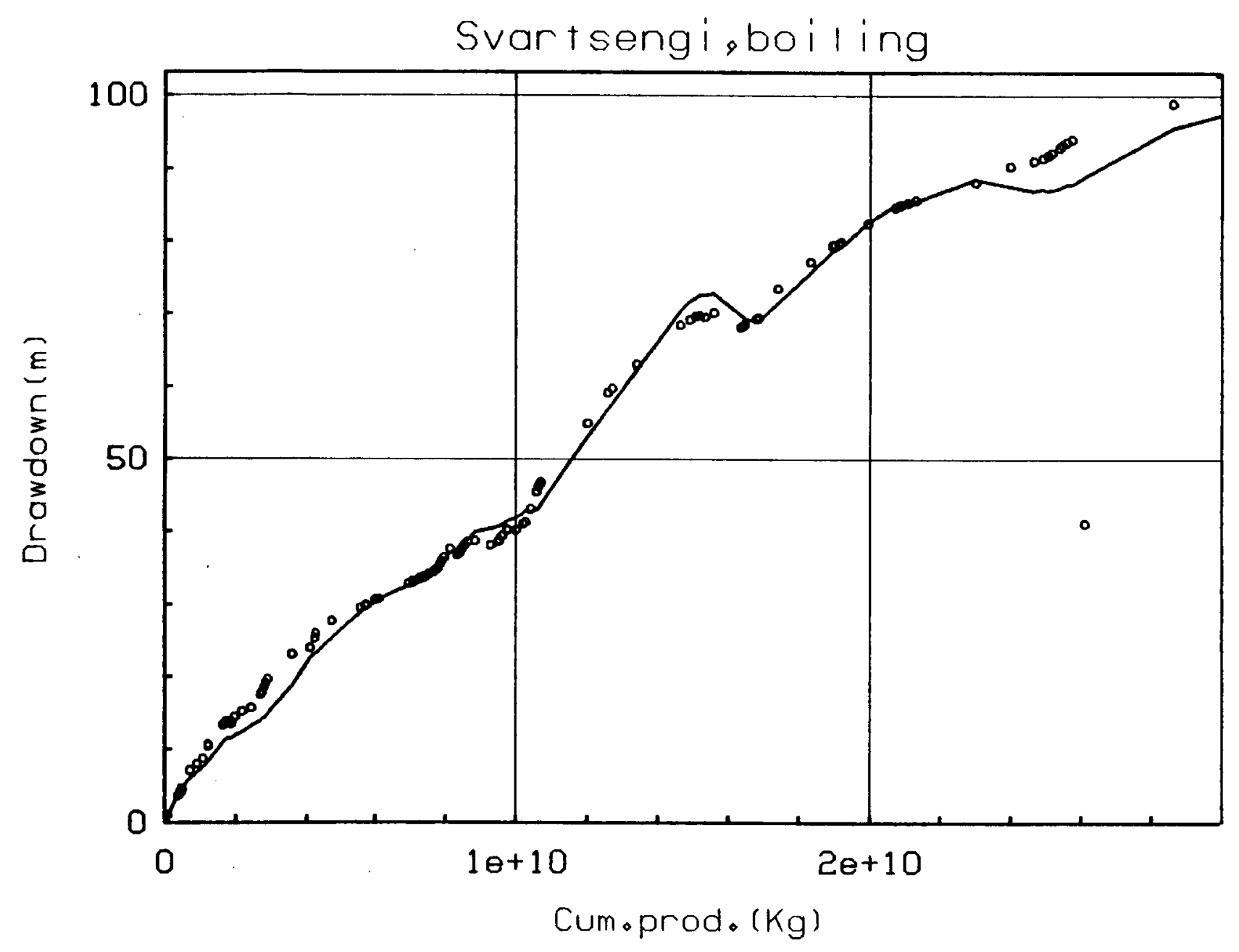

FIGURE-23. Measured and calculated drawdown using the boiling model with the constants from our fit. 


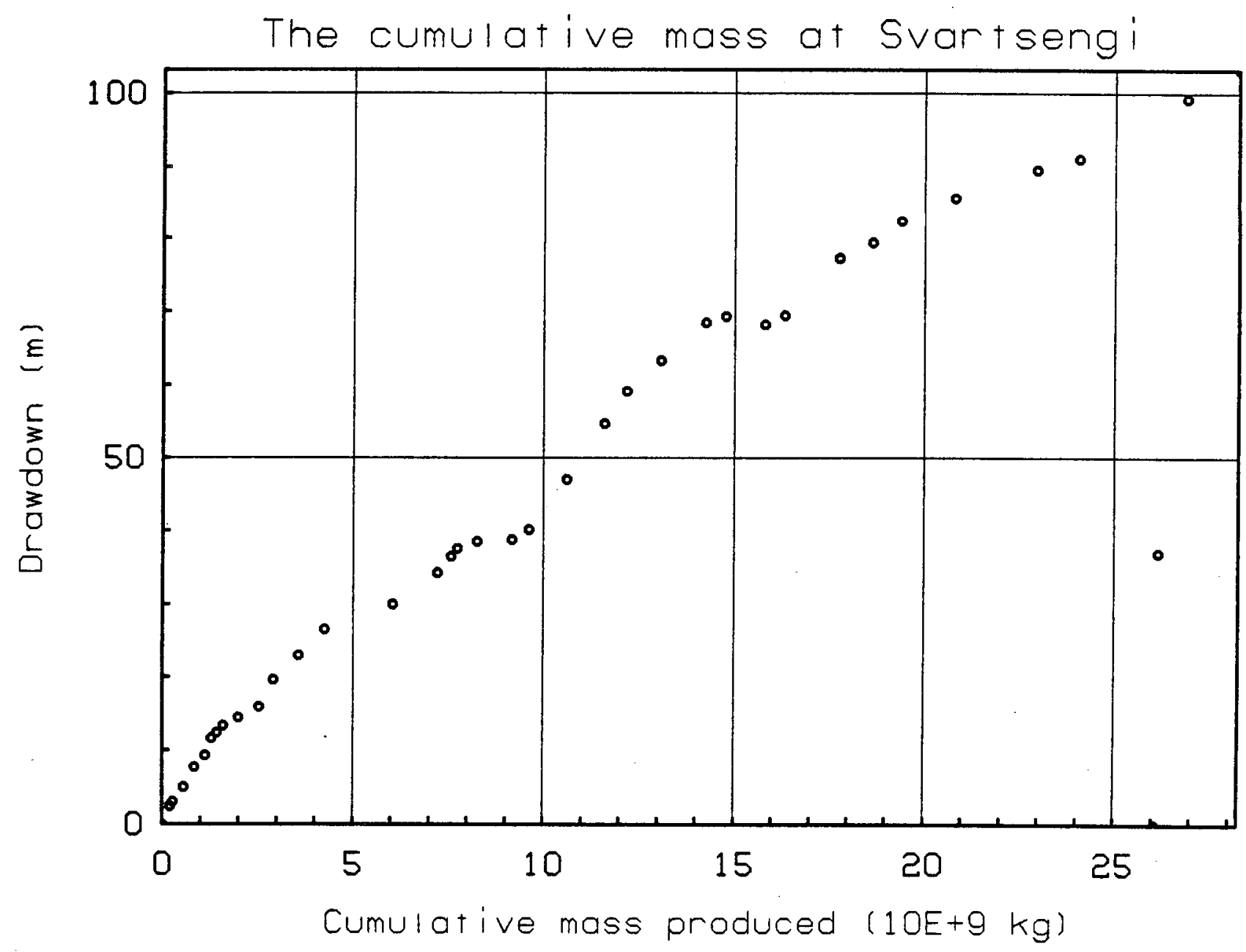

FIGURE-24. Drawdown vs. cumulative mass produced at Svartsengi. 


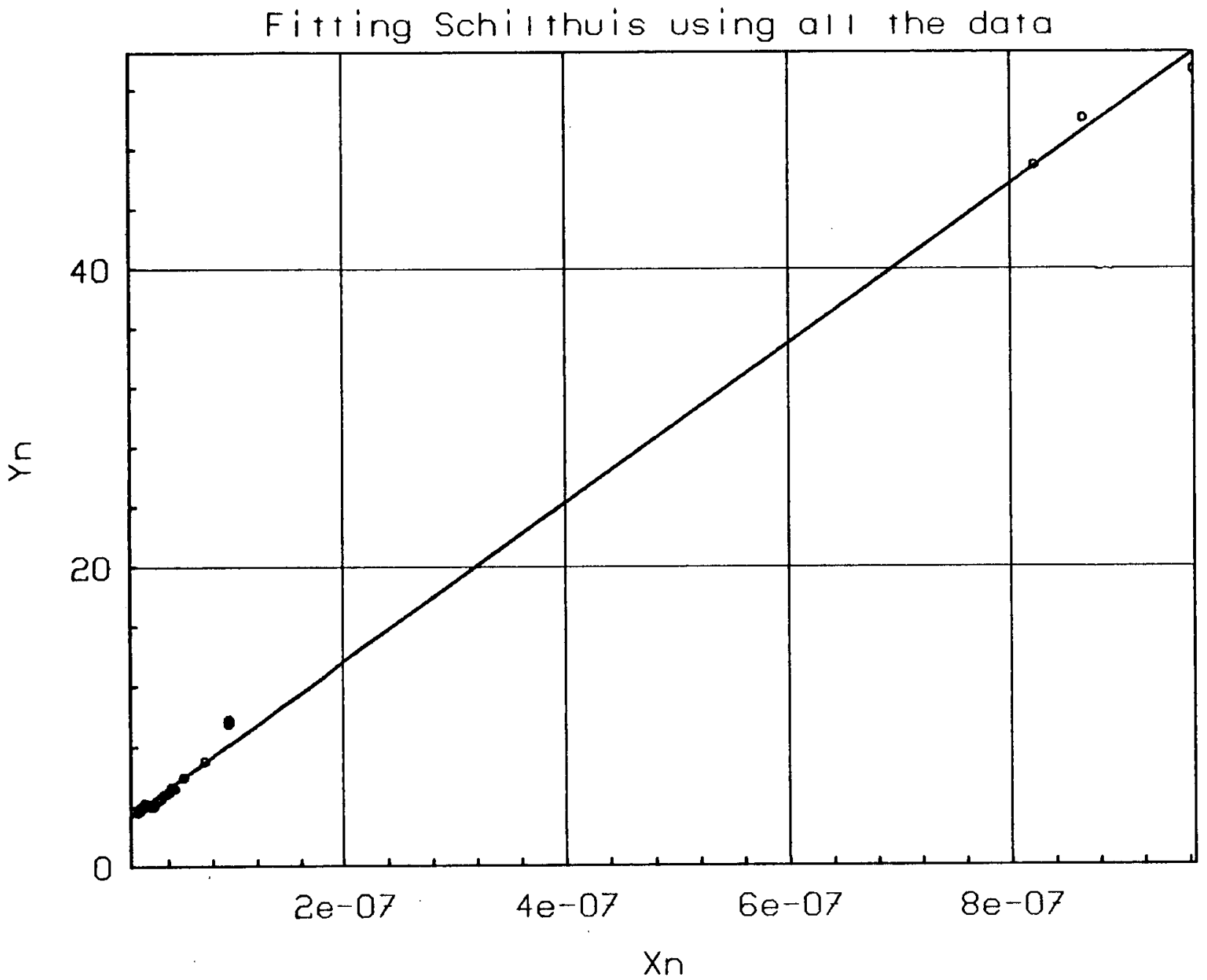

FIGURE-25. Matching the Schilthuis model using all the data. 


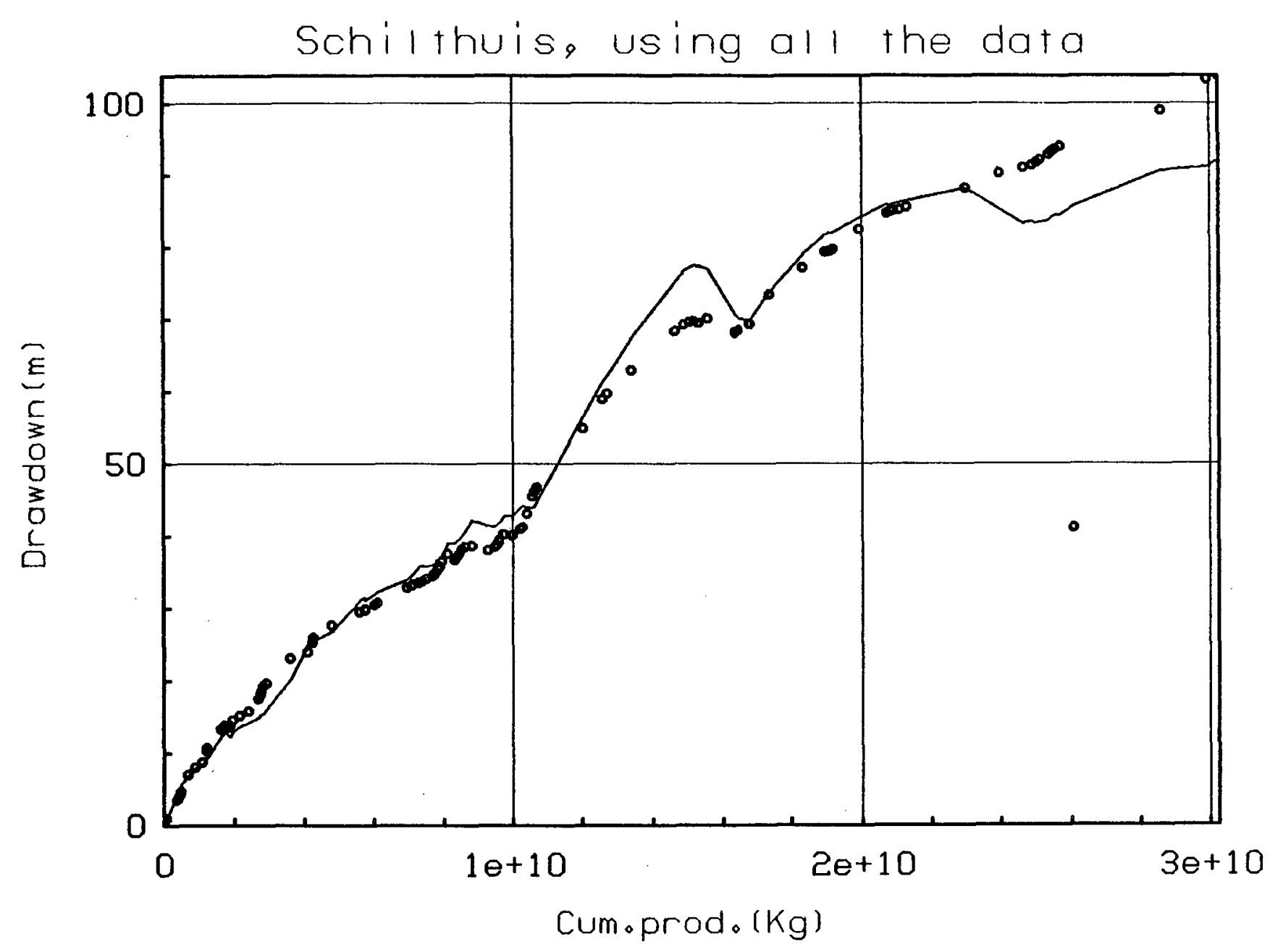

FIGURE-26. Measured and calculated drawdown for the Schilthuis model using all the data. 


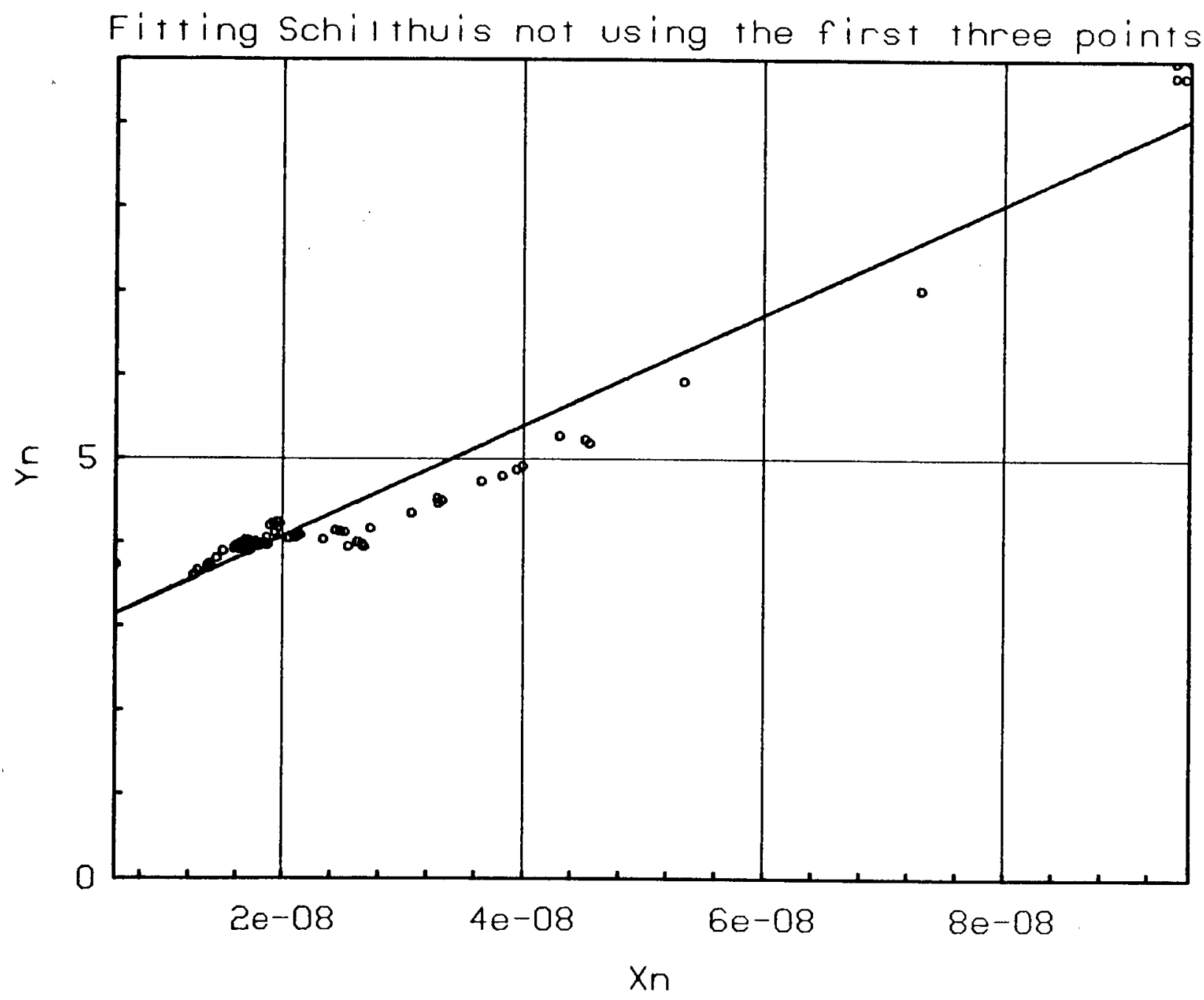

FIGURE-27. Matching the Schilthuis model without the first three points. 


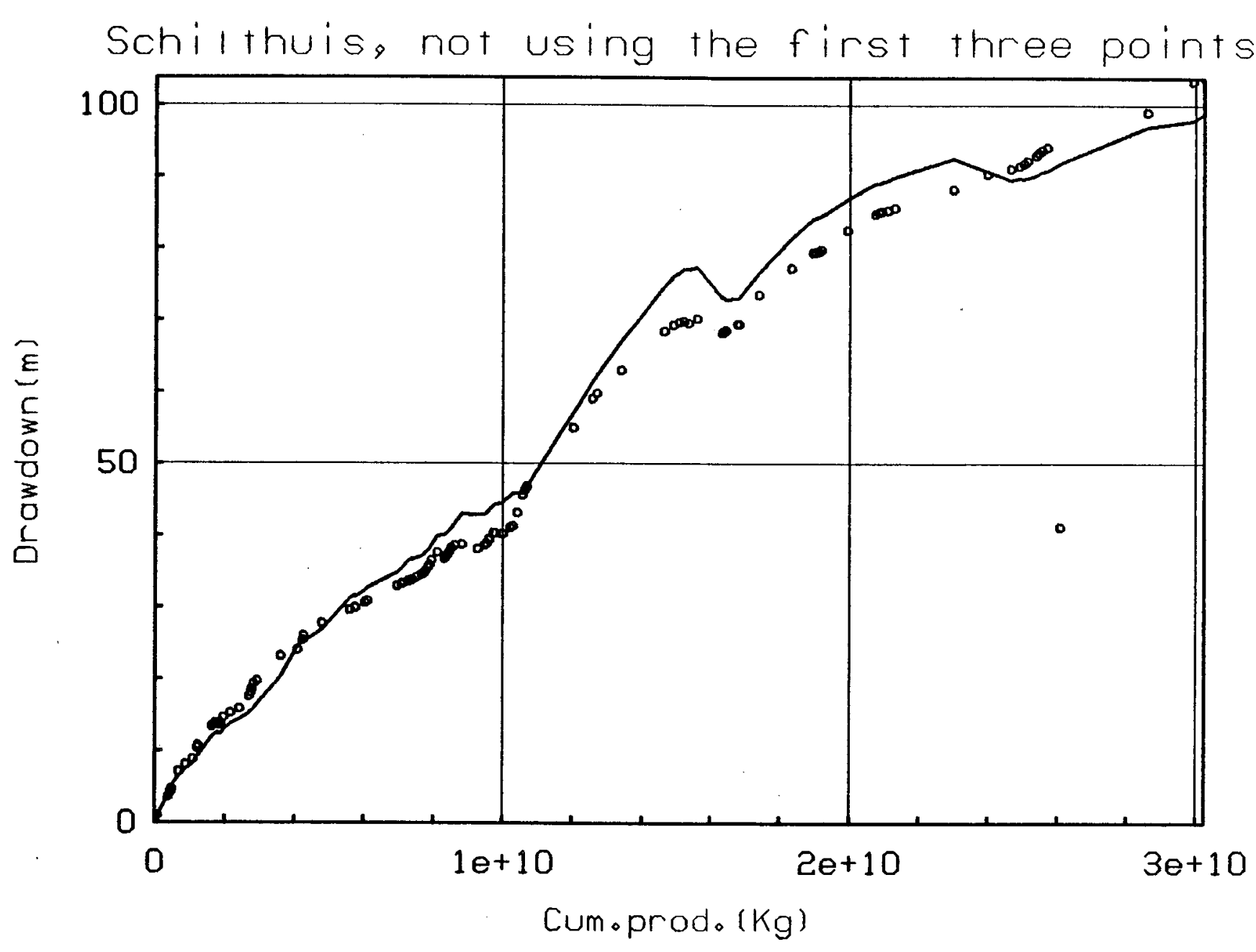

FIGURE-28. Measured and calculated drawdown for the Schilthuis model without using the first three points. 


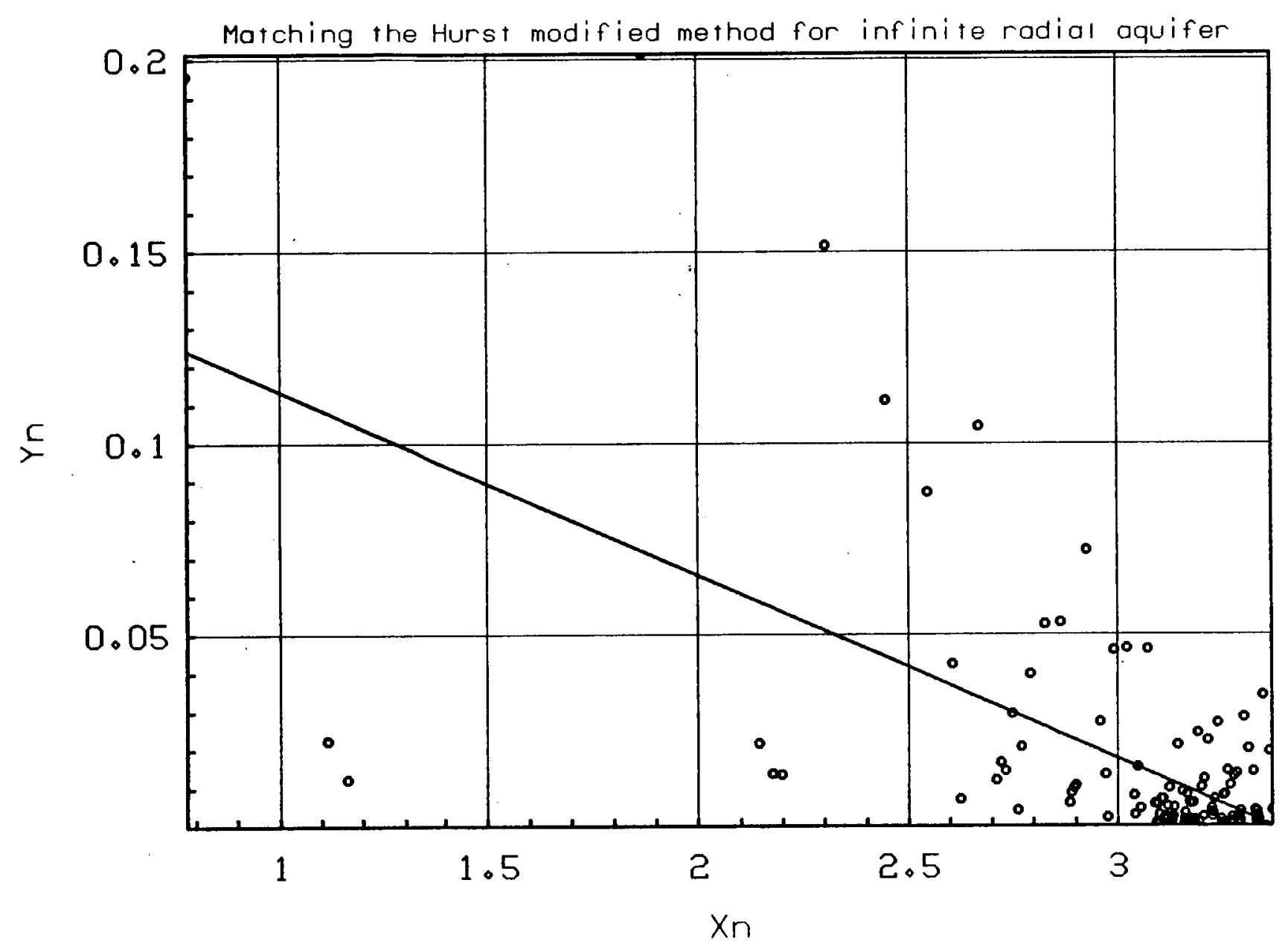

FIGURE-29. Fitting for $1 / a \log (b)$ and $1 / a$ in the Hurst modified method. 


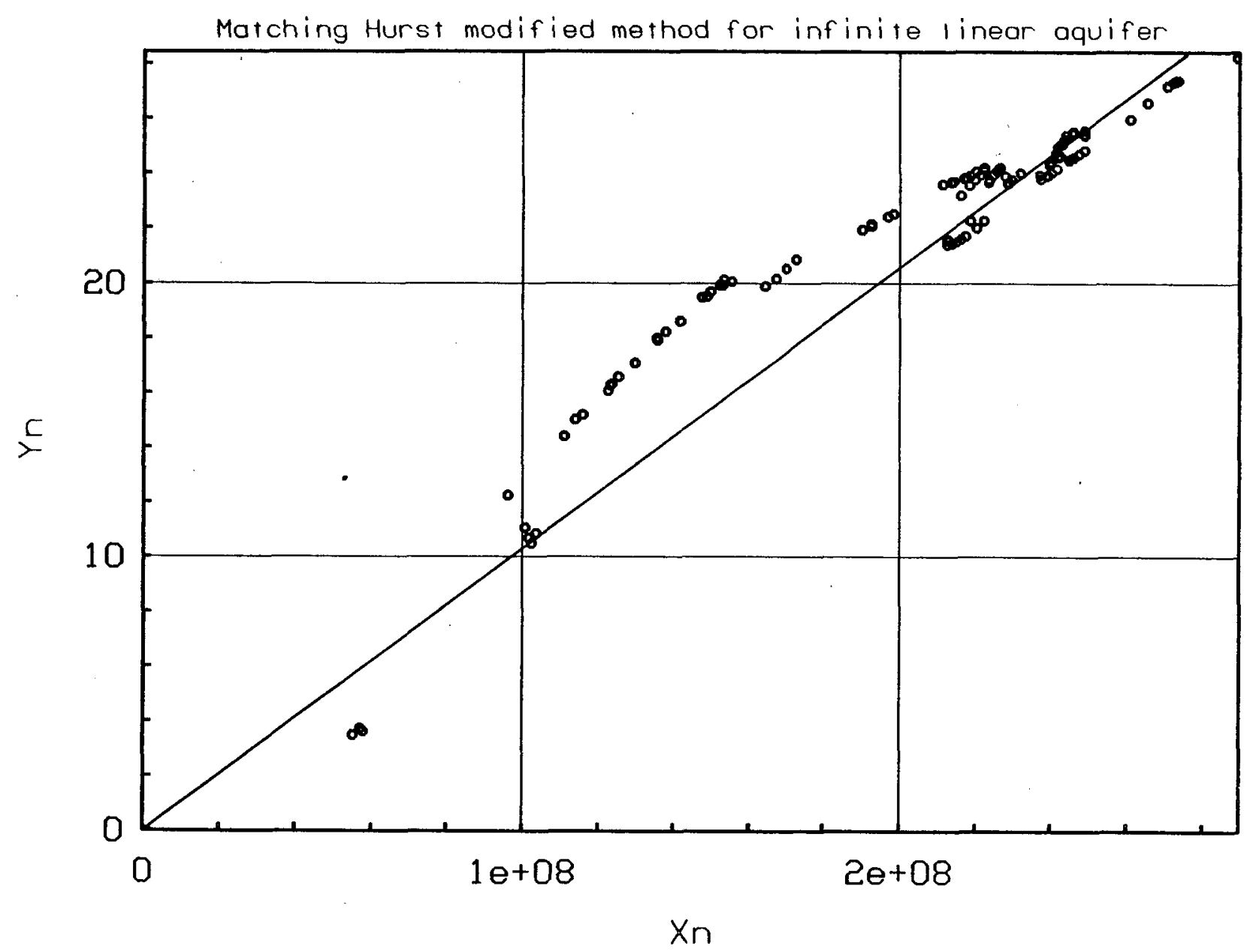

FIGURE-30. Matching the Hurst modified method with infinite linear aquifer. 


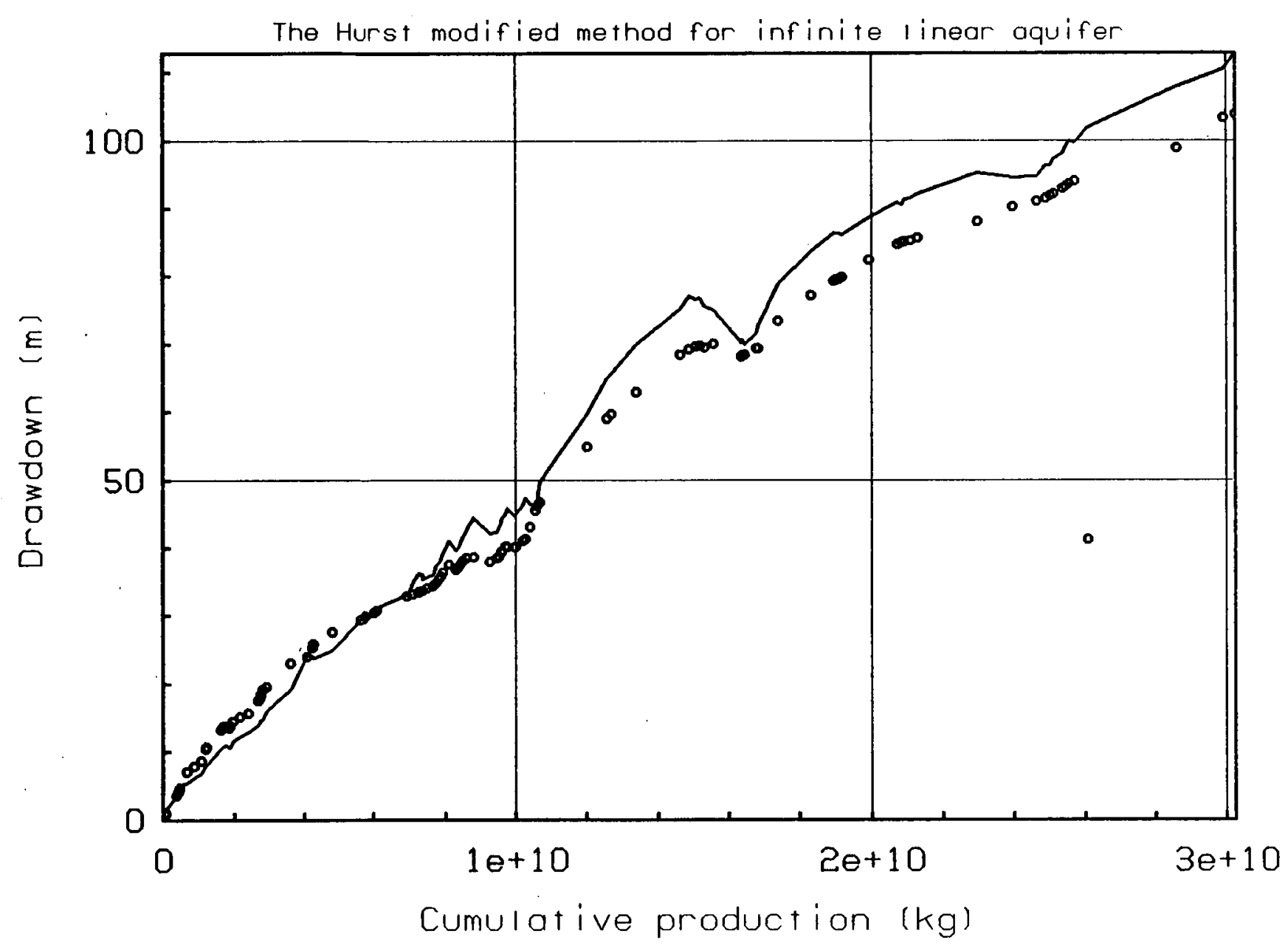

FIGURE-31. Measured and calculated drawdown for the Hurst modified method with infinite linear aquifer. 


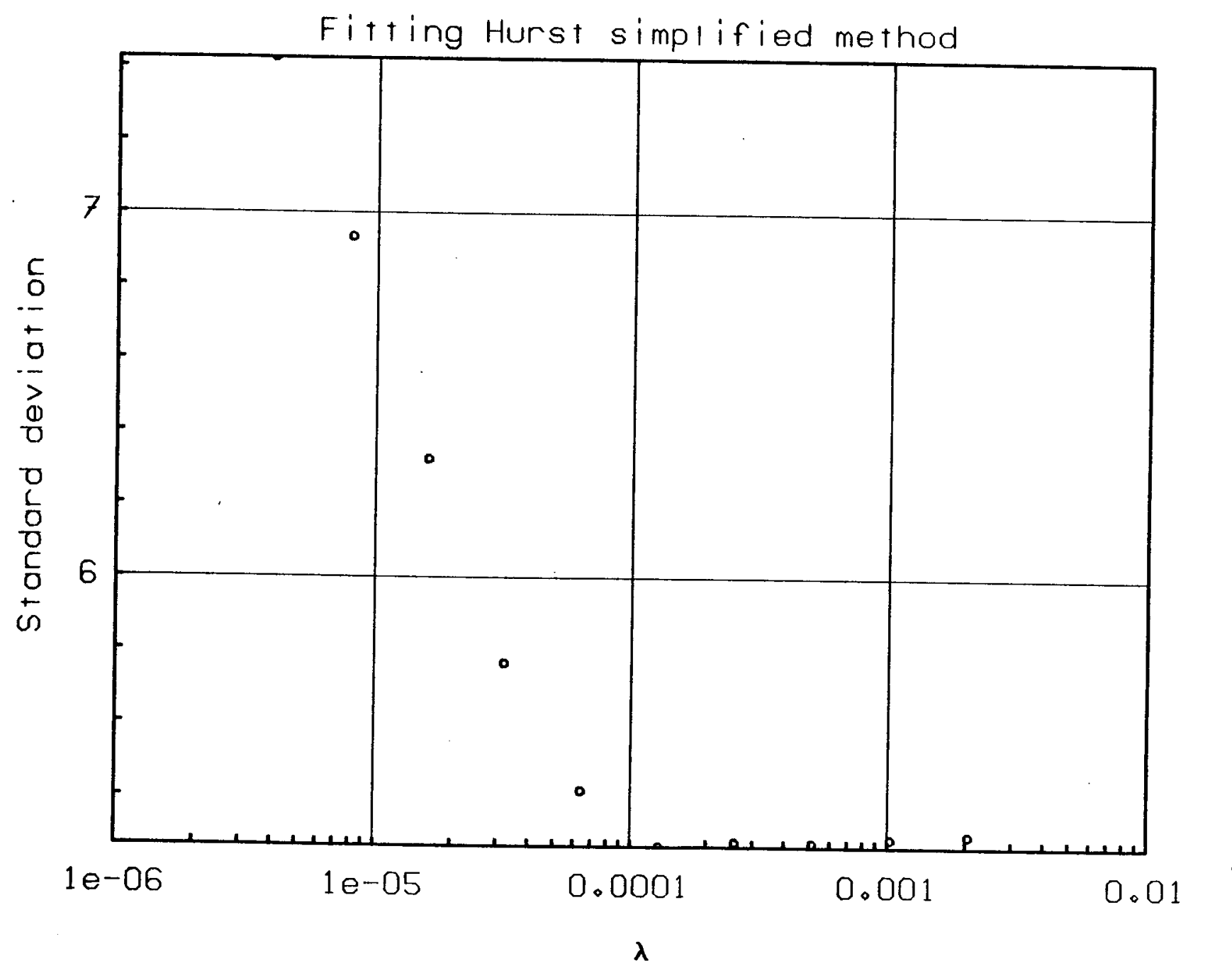

FIGURE-32. Determination of $\lambda$ for the Hurst simplified method. 
Hurst simplified model

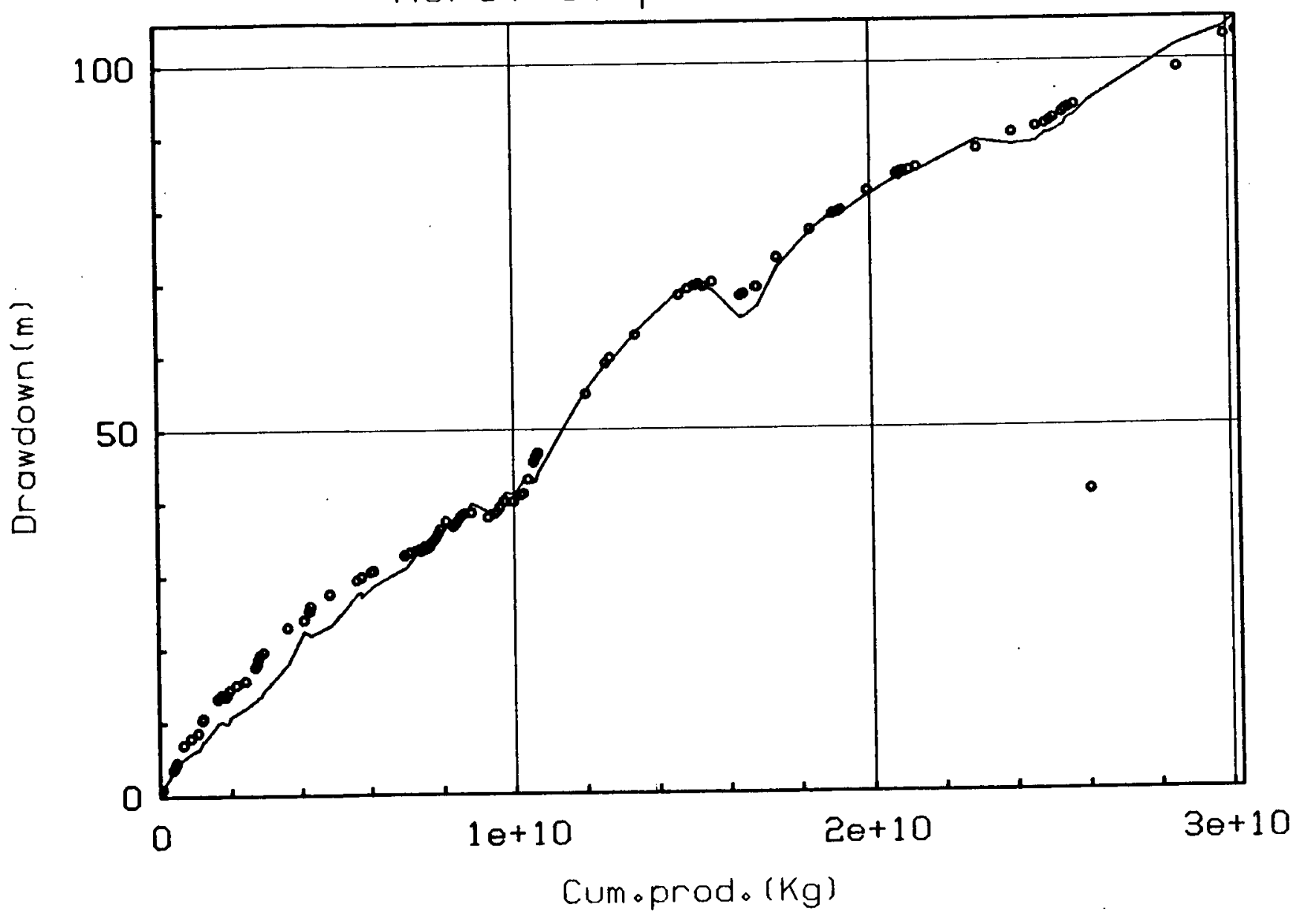

FIGURE-33. Measured and calculated drawdown for the Hurst simplified method. 
Finite aquifer, Fetkovitch

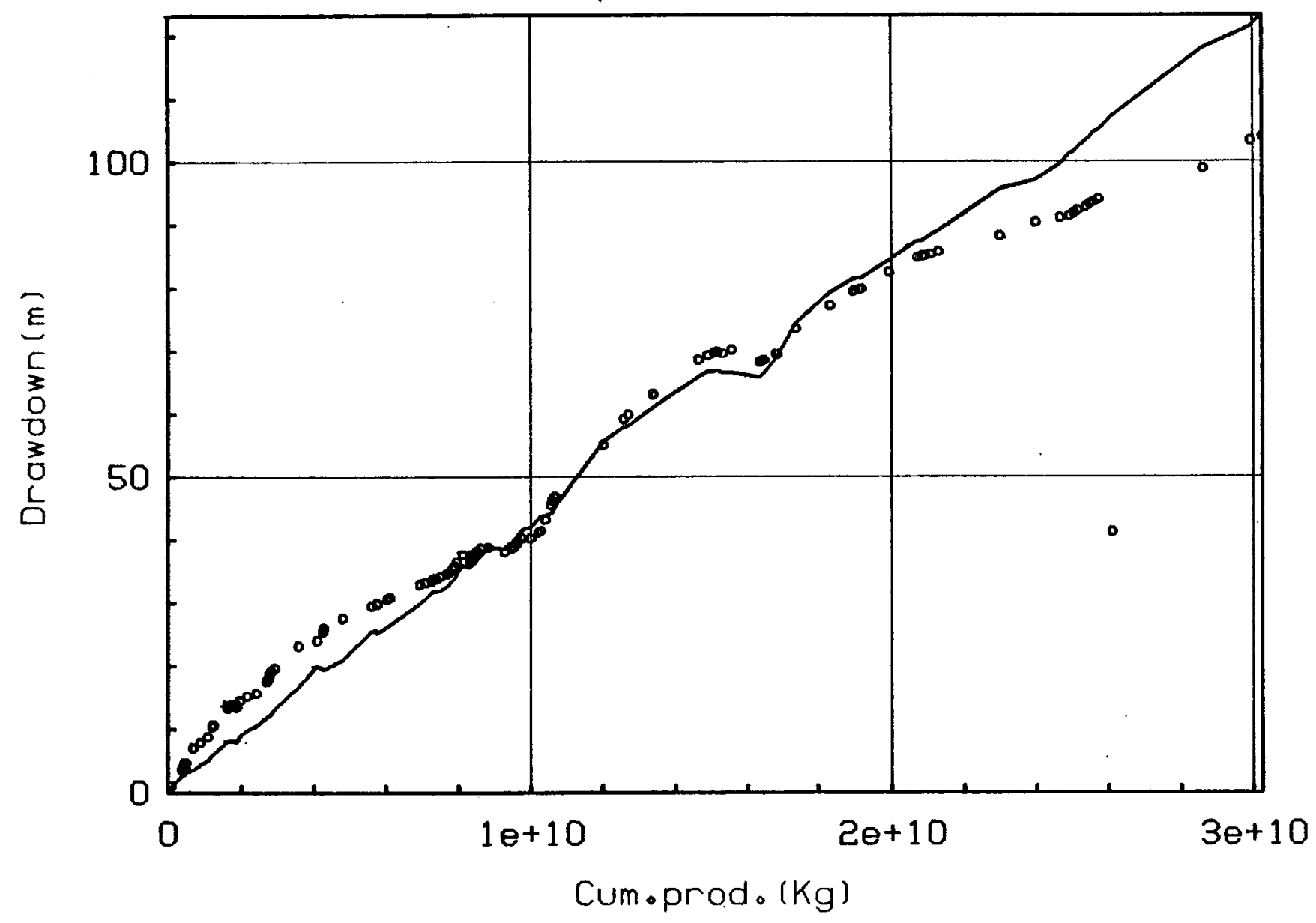

FIGURE-34. Measured and calculated drawdown for a finite linear aquifer using the Fetkovitch method. 
Fetkovitch,radial

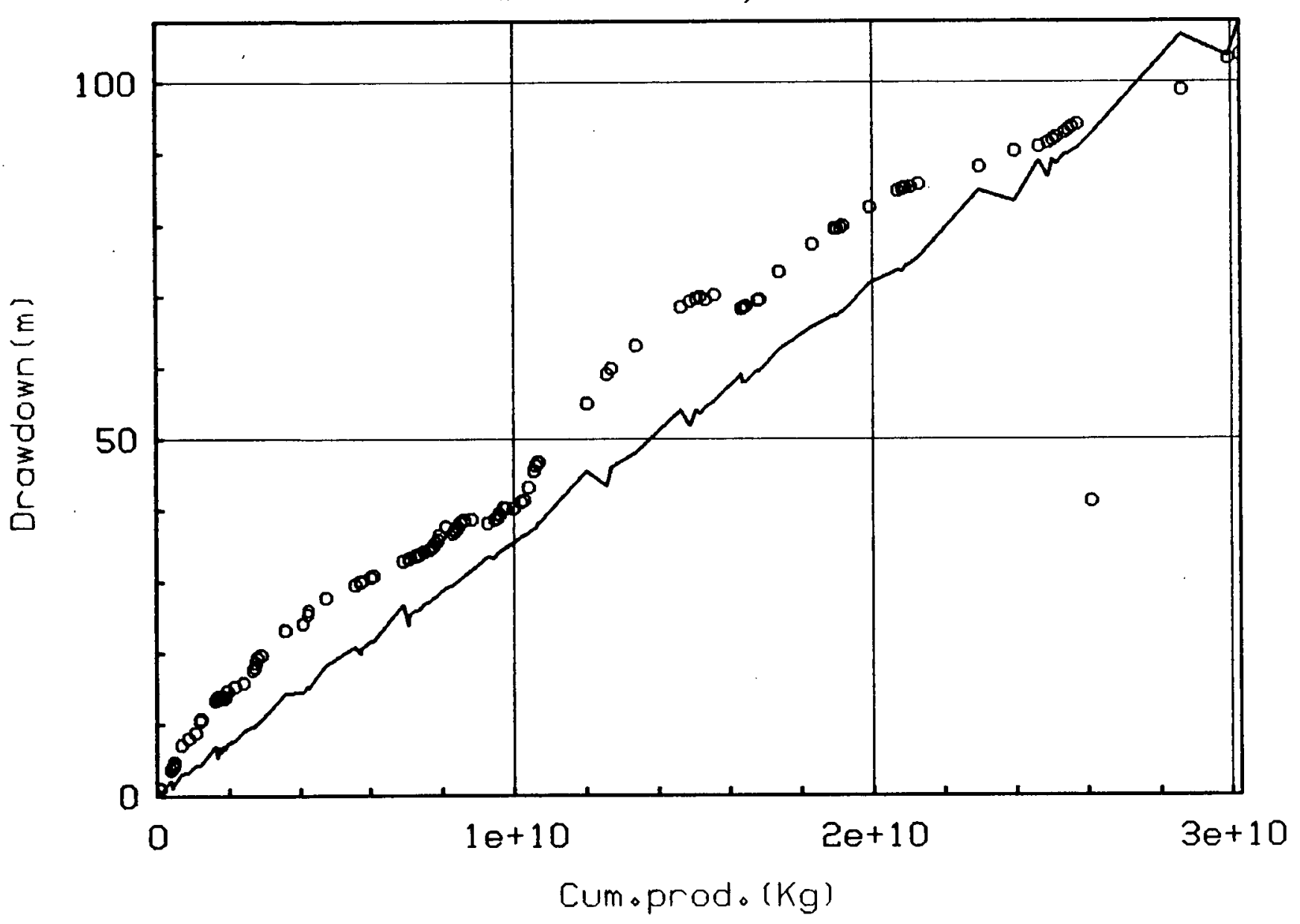

FIGURE-35. Measured and calculated drawdown for a finite radial aquifer using the Fetkovitch method. 


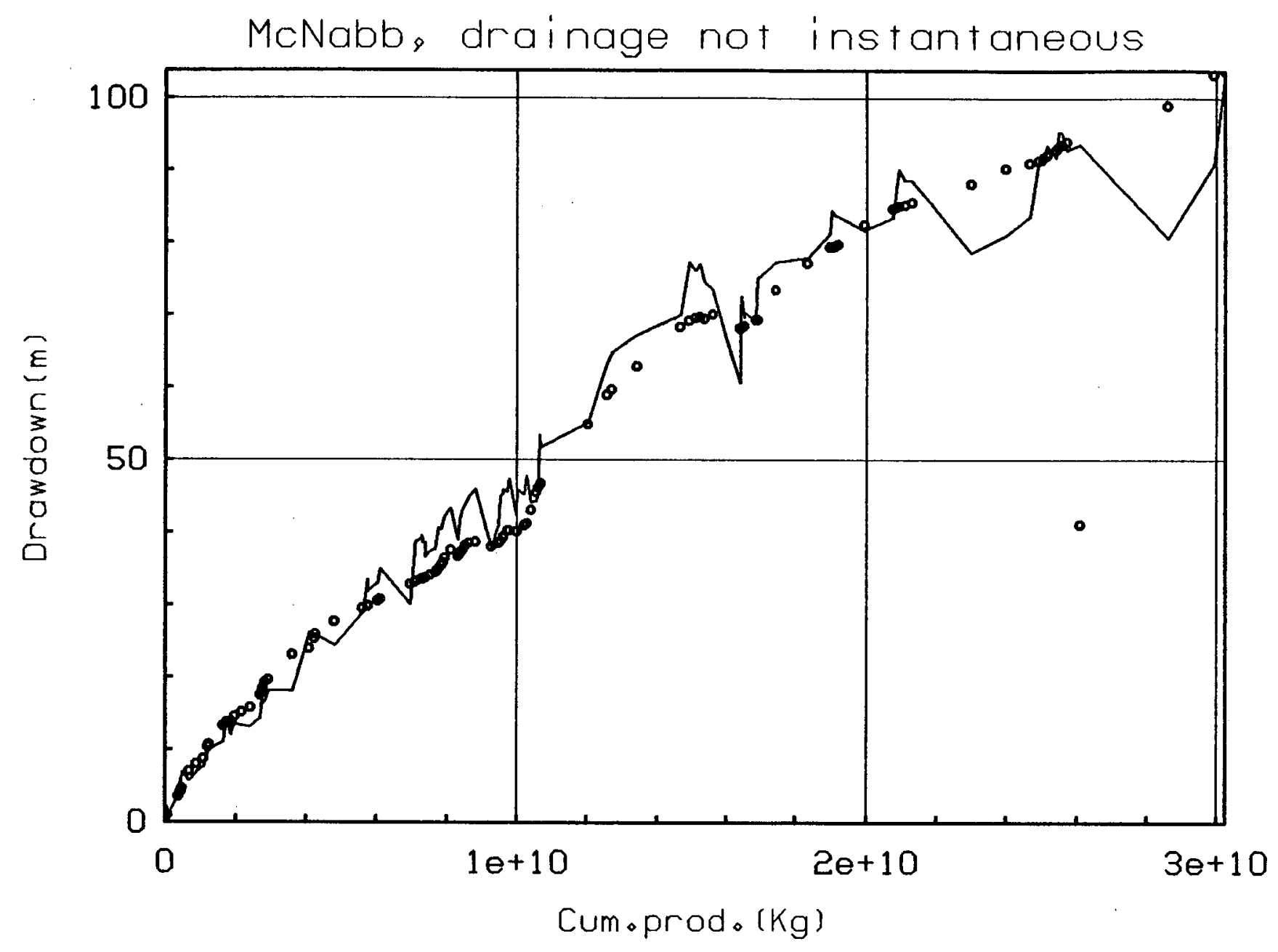

FIGURE-36. Measured and calculated drawdown if the drainage from the twophase zone is not instantaneous. The McNabb method. 


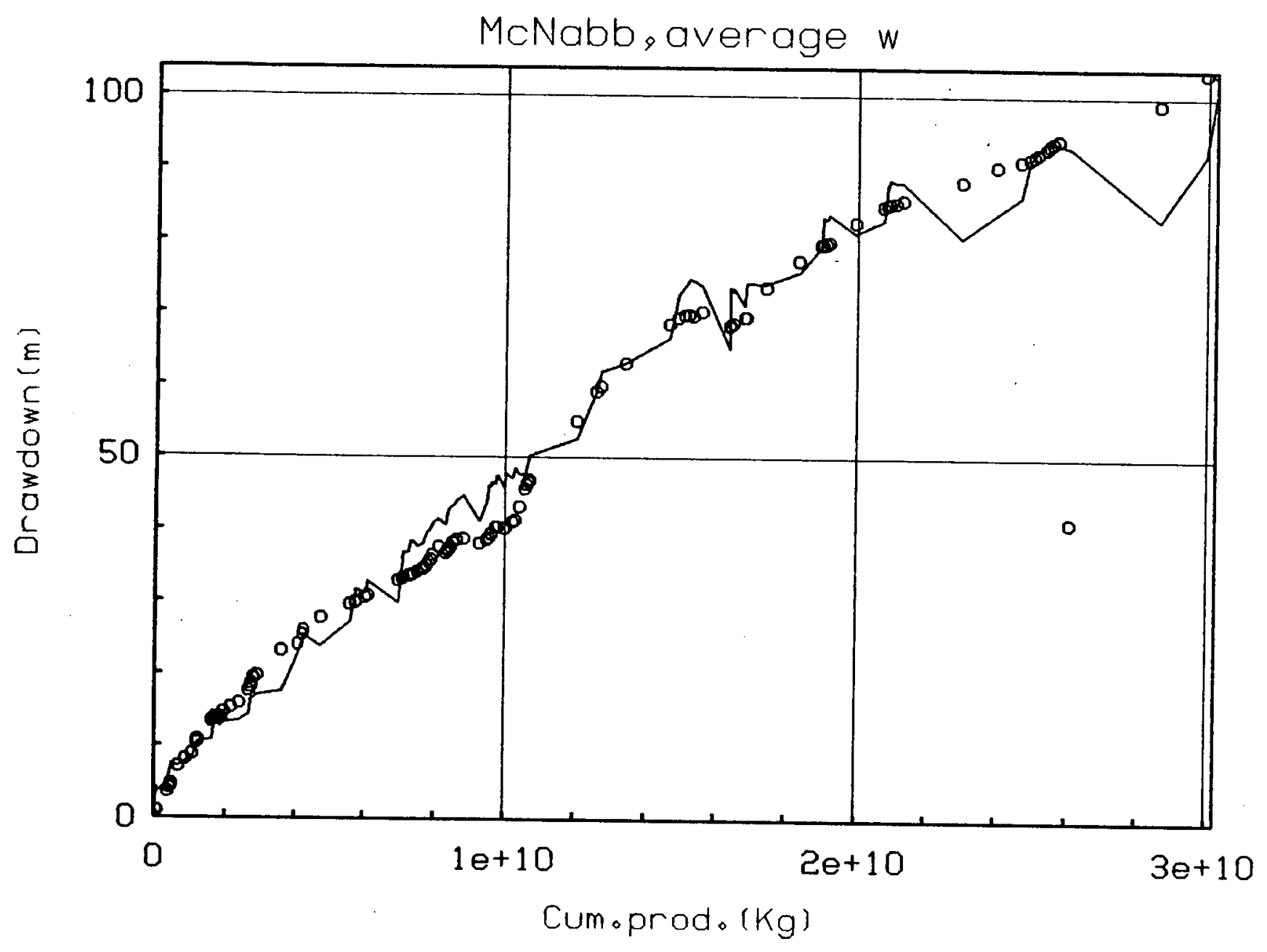

FIGURE-37. Same model as in Fig.36, but using average rate. 


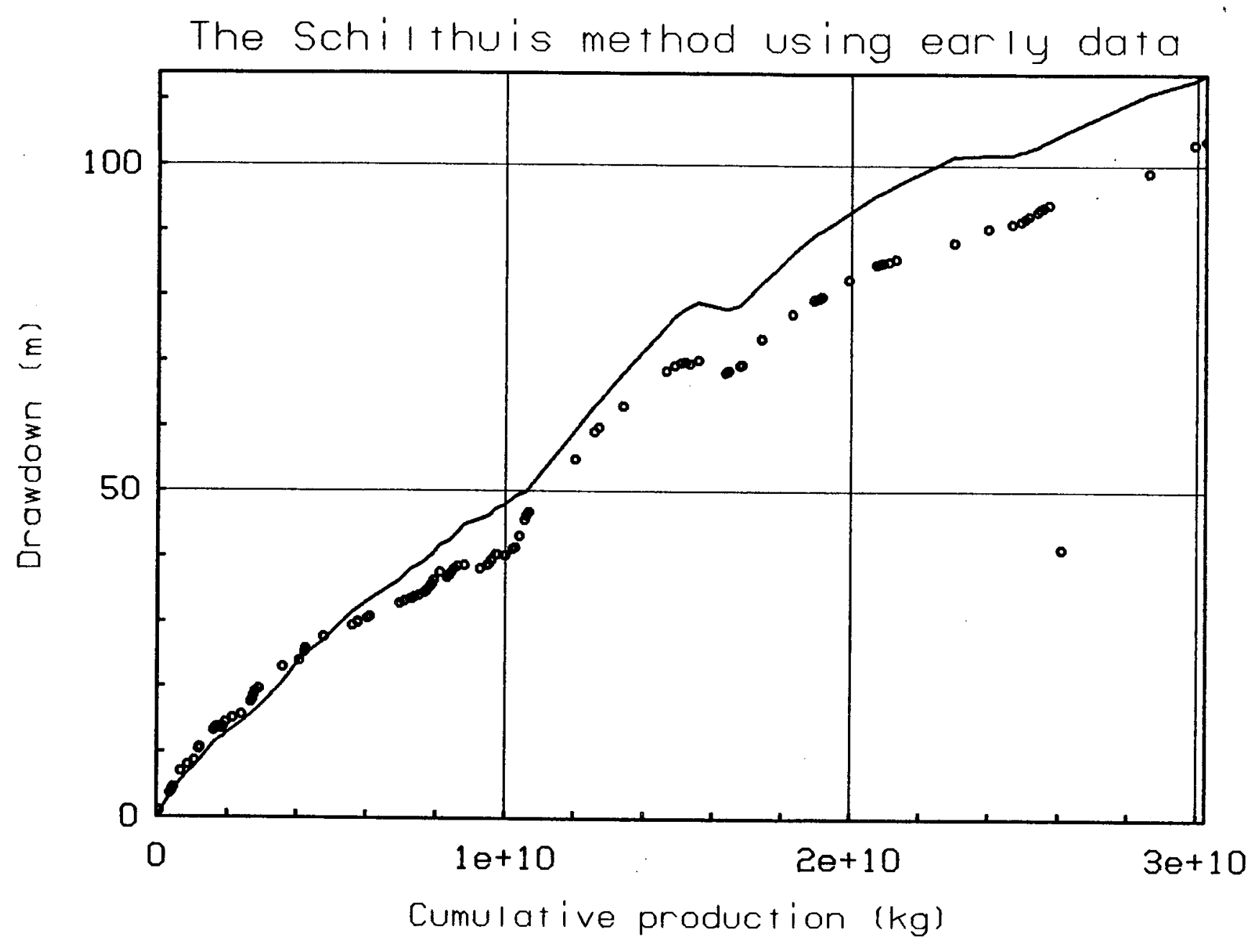

FIGURE-38. Measured and calculated drawdown for the Schilthuis method based on early time data. 


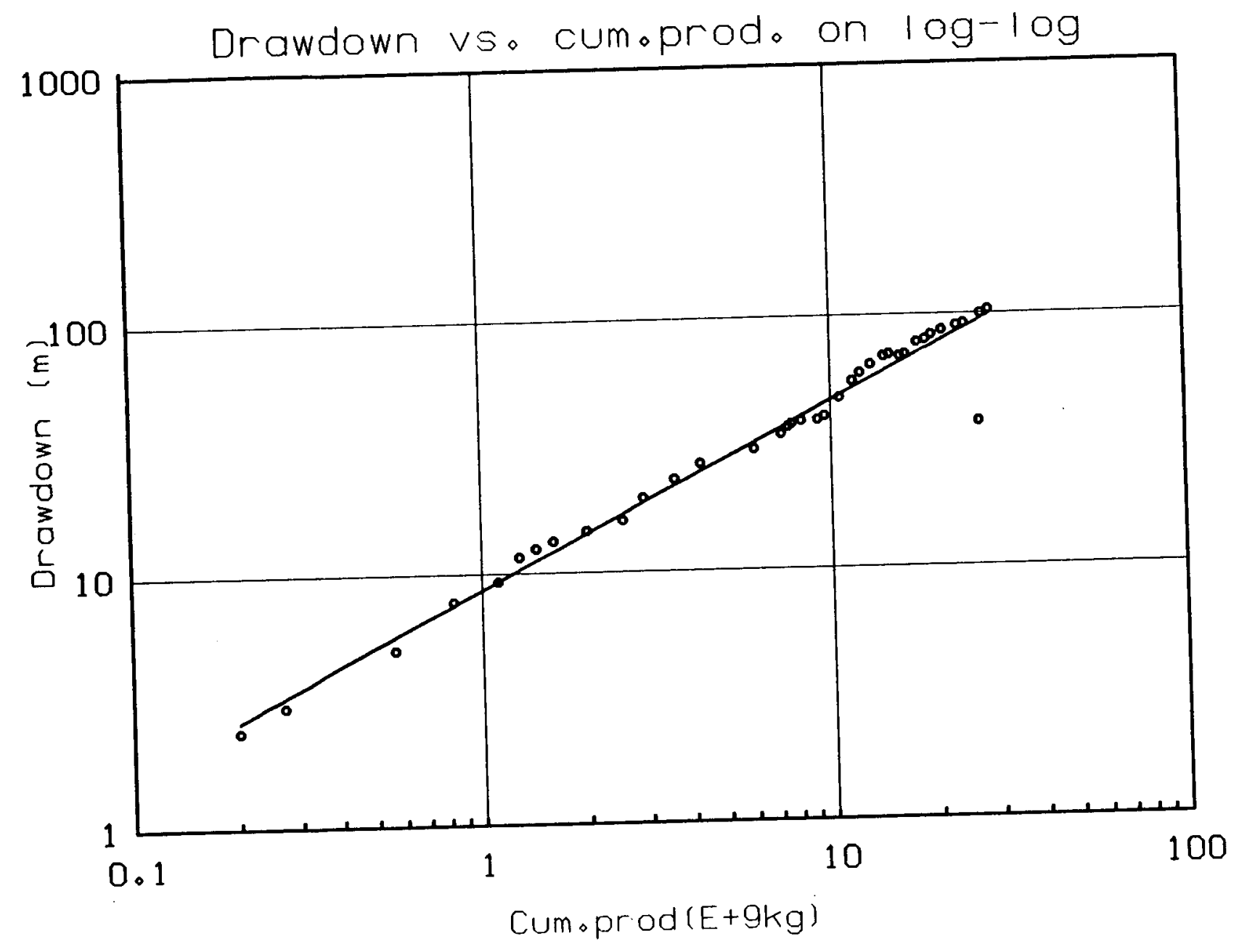

FIGURE-39. Log-log plot of measured drawdown vs. cumulative mass production. 


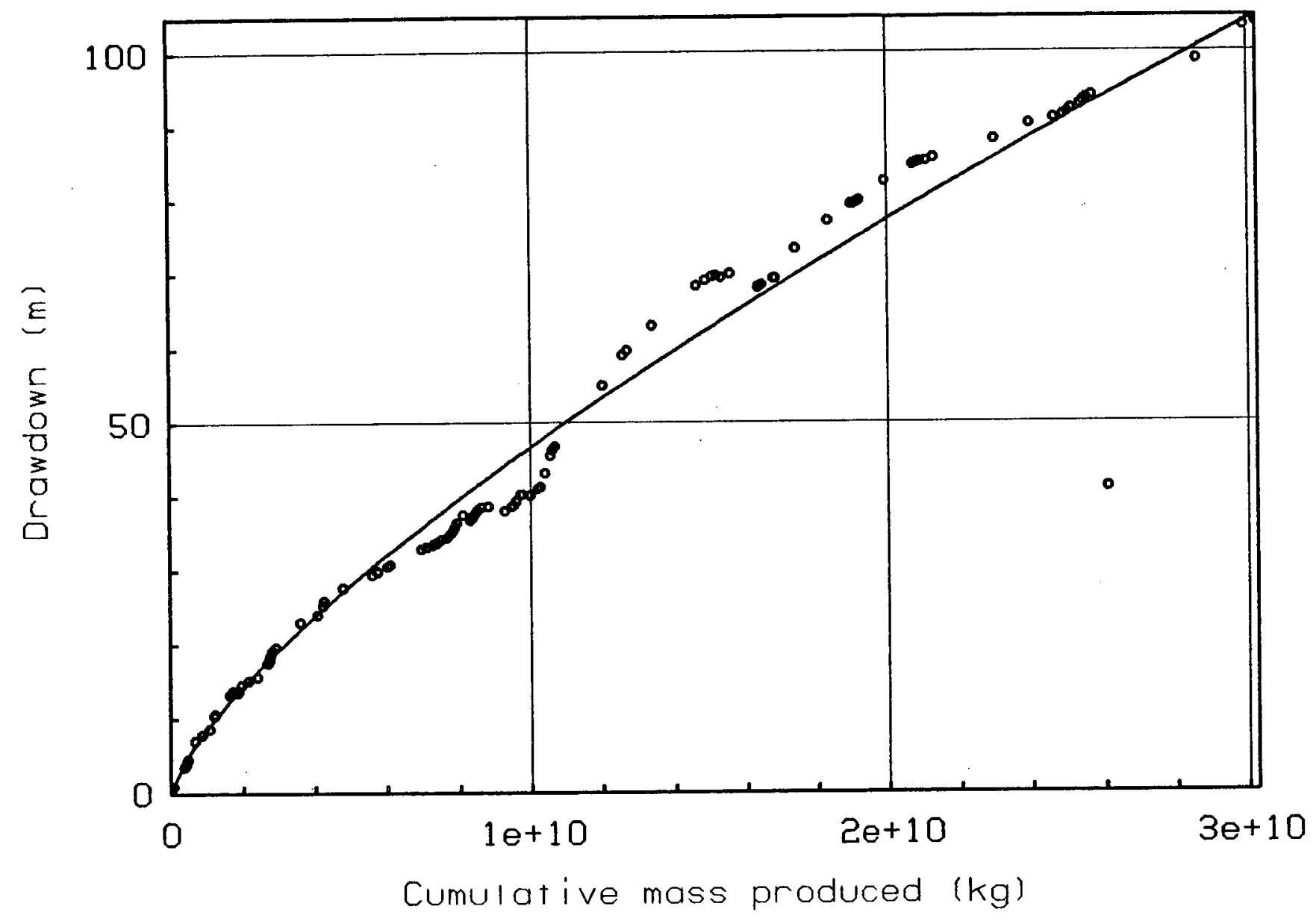

FIGURE-40. Measured and calculated drawdown for the log-log method. 


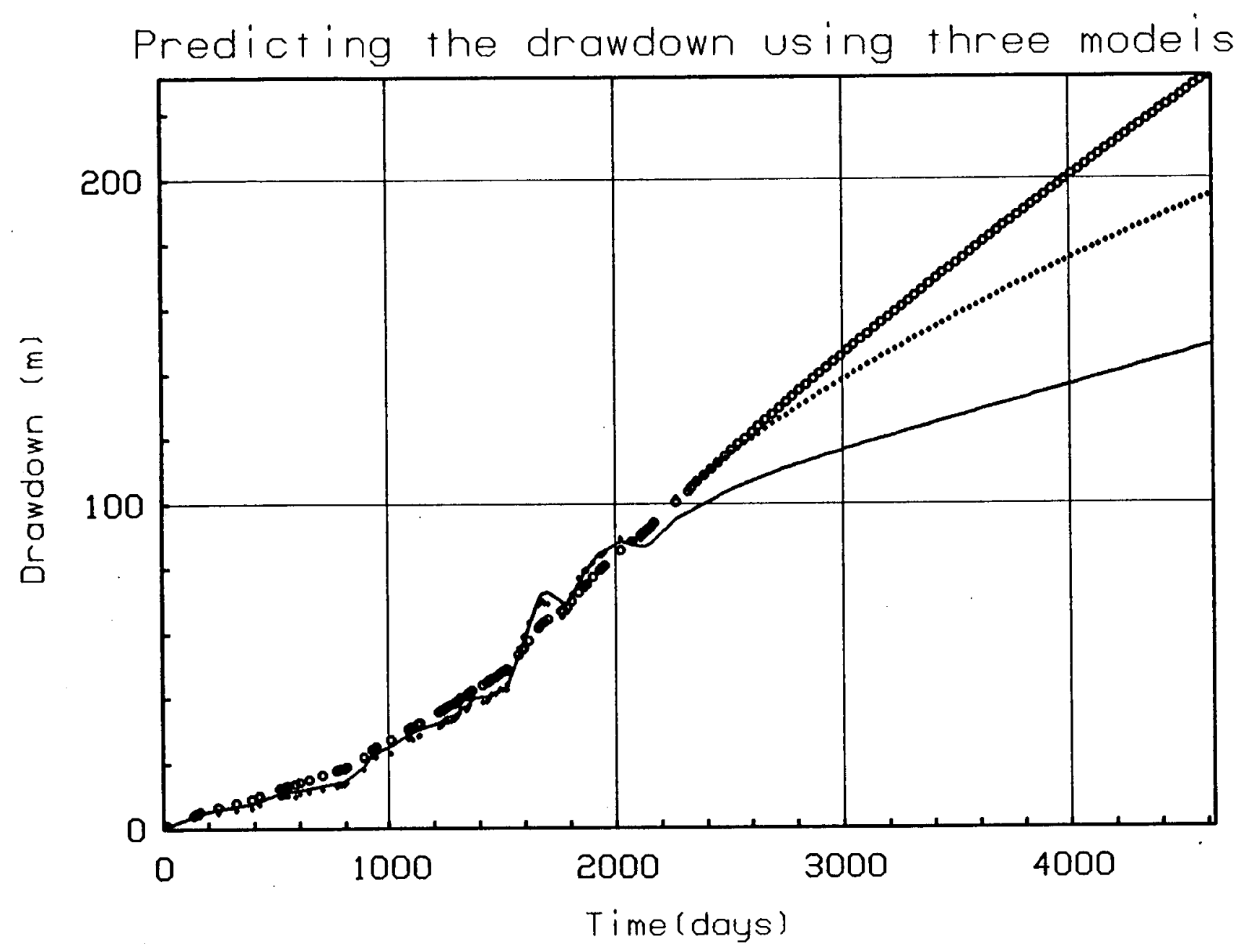

FIGURE-41. Comparison of predicted drawdown for three models. The circles represent the log-log model, the dots represent the Hurst simplified method, and the solid line represents the boiling model for Svartsengi. 


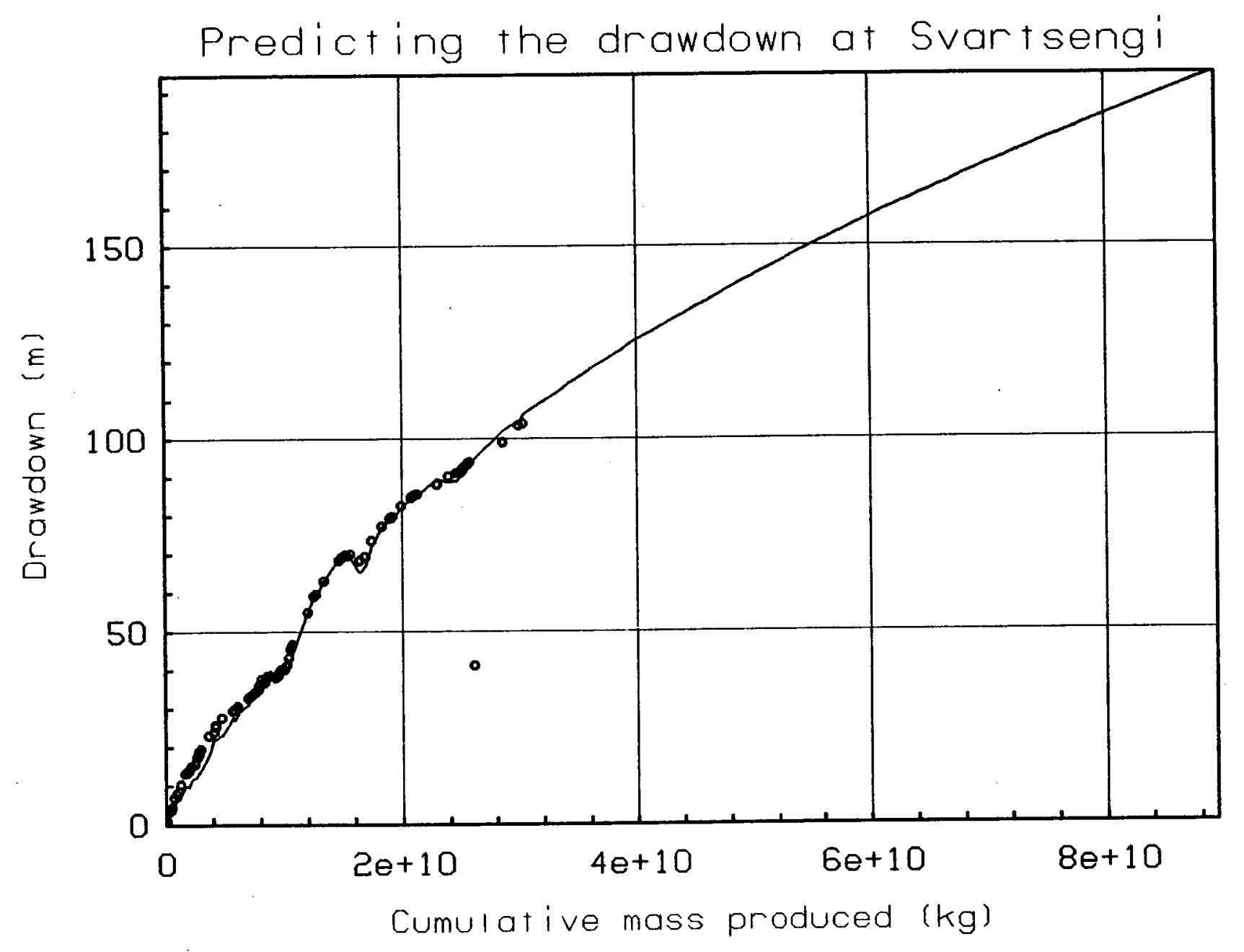

FIGURE-42. Prediction of the drawdown vs. cumulative production for a constant rate of $300 \mathrm{~kg} / \mathrm{s}$ using the Hurst simplified method. 


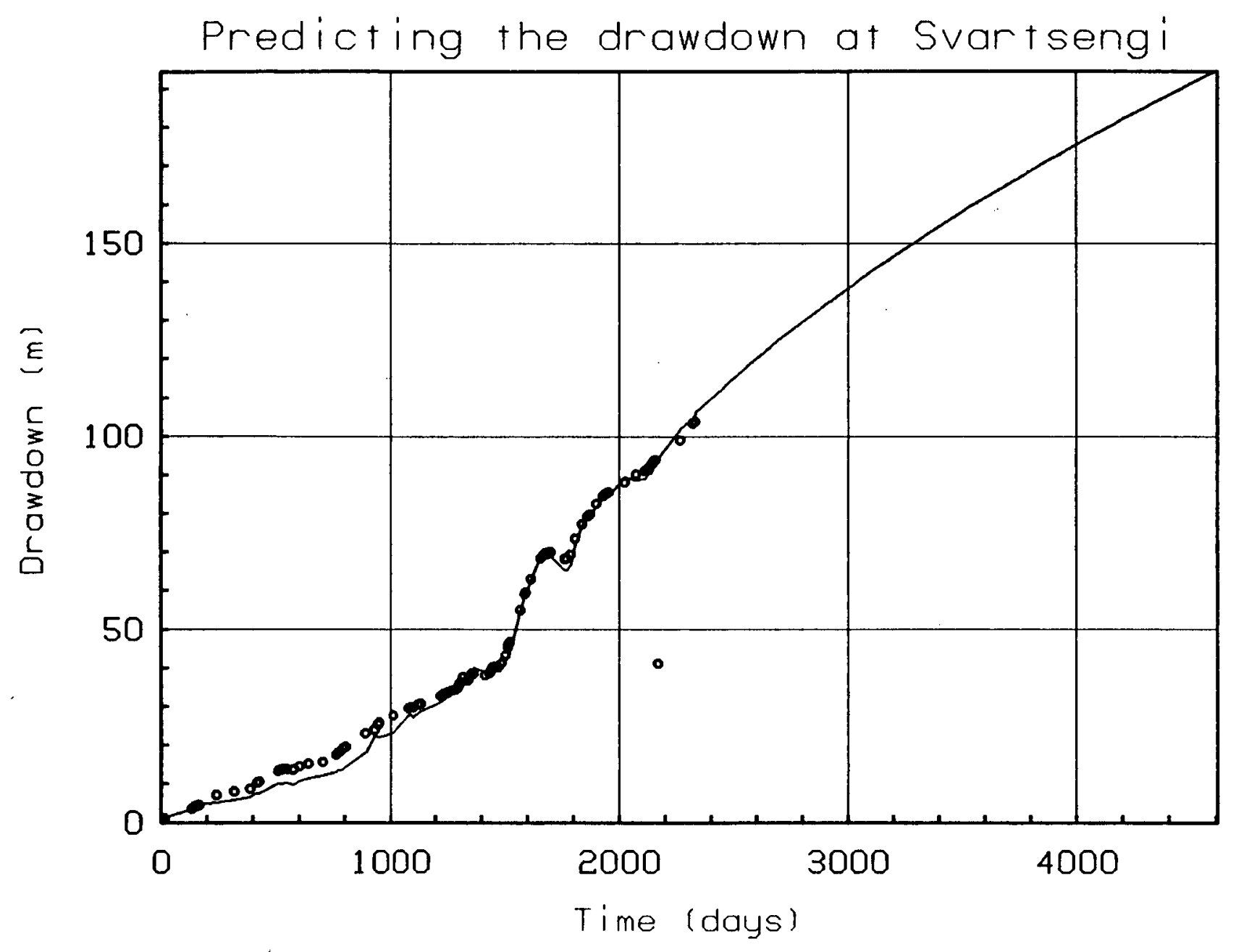

FIGURE-43. Prediction of the drawdown vs. time for a constant rate of $300 \mathrm{~kg} / \mathrm{s}$ using the Hurst simplified method. 


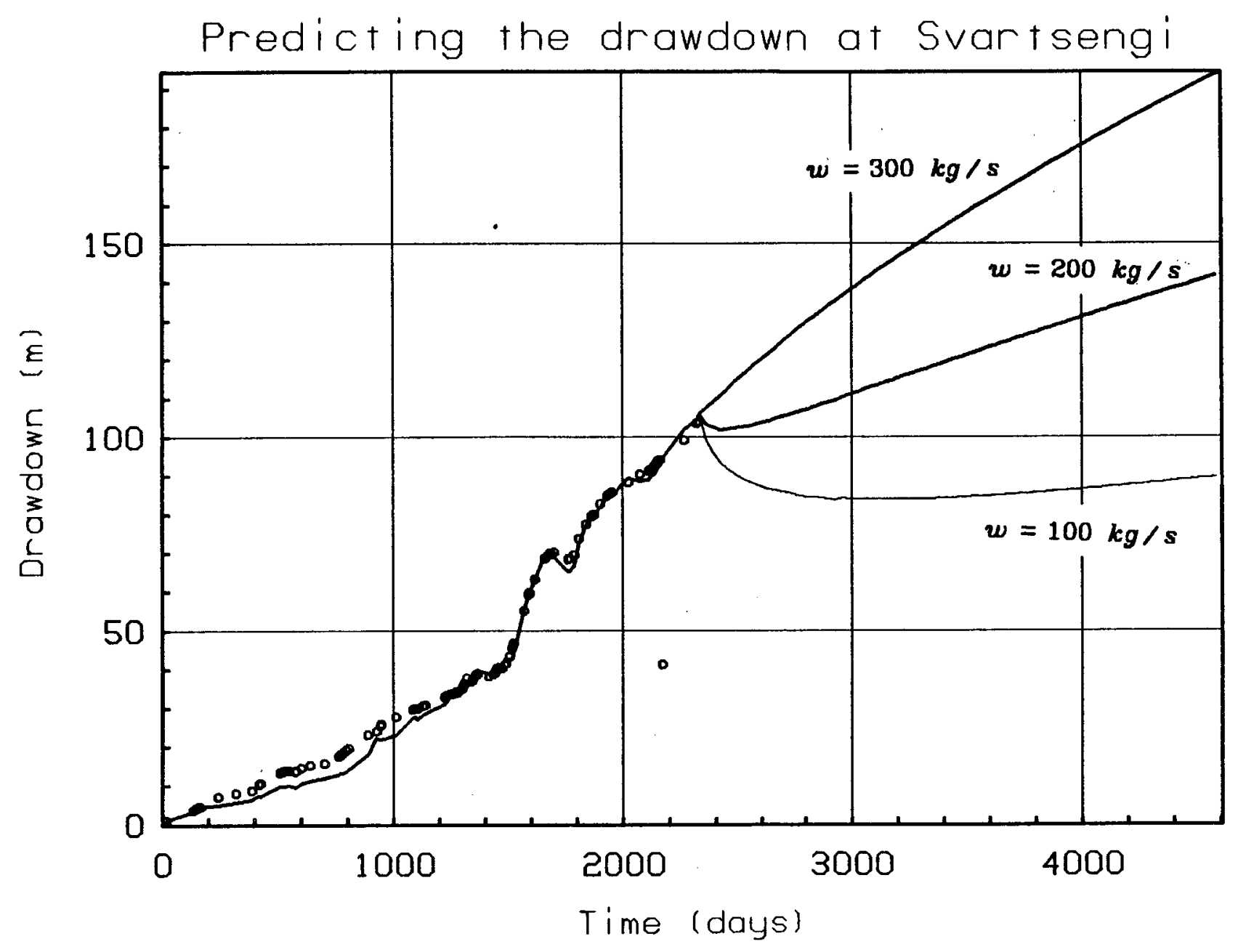

FIGURE-44. Prediction of the drawdown vs. time for three different rates using the Hurst simplified method. 


\section{APPENDIX A: Derivations of Equations.}

\section{A. 1. Liquid Models}

The mass balance in the system is given by:

$$
W_{c}=W_{i}-W_{p}-W_{l}+W_{a}
$$

Assuming isothermal conditions, the heat balance is neglected. If we assume that $W_{l}$ can be neglected, (A-1) reduces to:

$$
W_{c}=W_{i}-W_{p}+W_{i}
$$

If the density is given by $\rho$, the mass is given by:

$$
\begin{aligned}
& W_{i}=V_{i} \varphi \rho_{i} \\
& W_{c}=V_{c} \varphi \rho_{c}
\end{aligned}
$$

\section{A.1. Unconfined System}

In this case we assume that $\rho_{c}=\rho_{i}$, and therefore the volume must be changing:

$$
\begin{aligned}
V_{i} & =A_{i} h_{i} \\
V_{c} & =A_{c} h_{c}
\end{aligned}
$$

Now if we assume that $A$ is constant with depth, using Eq.A-4 in Eq.A-3 gives:

$$
\begin{aligned}
& W_{i}=A h_{i} \varphi_{i} \rho \\
& W_{c}=A h_{c} \varphi_{c} \rho
\end{aligned}
$$

If we also assume that $\varphi$ is constant with depth, substituting Eq.A-5 in Eq.A-2 gives:

$$
A h_{c} \varphi \rho=A h_{i} \varphi \rho-W_{p}+W_{e}
$$

which simplifies to:

$$
A \varphi \rho\left(h_{c}-h_{i}\right)=-W_{p}+W_{e}
$$

which simplifies to:

$$
A \varphi \rho \frac{d h}{d t}=\frac{d W_{d}}{d t}-\frac{d W_{p}}{d t}
$$

Now $d W_{0} / d t=w_{e}$, the mass influx rate, and $d W_{p} / d t=w_{p}$, the mass production 
rate. Therefore Eq.A-8 becomes:

$$
A \varphi \rho \frac{d h}{d t}=w_{\theta}-w_{p}
$$

At this point several influx models are possible, and have been discussed in the text. Since we assume $\rho$ constant, the pressure is given by the hydrostatic column of liquid water. The pressure at level $h$ in the reservoir is given by:

$$
\boldsymbol{p}=\rho g h
$$

differentiating Eq.A-10 with respect to time gives:

$$
\begin{aligned}
& \frac{d p}{d t}=\rho g \frac{d h}{d t} \\
& \frac{d h}{d t}=\frac{1}{\rho g} \frac{d p}{d t}
\end{aligned}
$$

Substituting Eq.A-11b in Eq.A-9 gives:

$$
\frac{A \varphi}{g} \frac{d p}{d t}=w_{e}-w_{p}
$$

\section{A 1.2. Confined System}

Here we assume that the volume is constant, and that the fluids expand into . the space created by the produced fluid. Substituting Eq.A-3 in Eq.A-2 gives:

$$
V \varphi \rho_{c}=V \varphi \rho_{i}-W_{p}+W_{e}
$$

Differentiating Eq.A-13 with respect to time gives:

$$
V \varphi \frac{d \rho}{d t}=w_{a}-w_{p}
$$

If we assume that the change of density with pressure is constant (this is only a good approximation for slightly compressible liquids, and for small changes in pressure), Eq.A-14 may be written:

$$
V \varphi\left(\frac{d \rho}{d p}\right)_{T} \frac{d p}{d t}=w_{e}-w_{p}
$$

\section{A.1.3. Compressibility of Liquid}

The isothermal compressibility is defined as:

$$
c=-\frac{1}{V}\left(\frac{\partial V}{\partial p}\right)_{T}
$$


The density is defined as:

$$
\rho=\frac{W}{V}
$$

Solving for $V$ gives:

$$
V=\frac{W}{\rho}
$$

Differentiating Eq.A-18 with respect to pressure, gives:

$$
\frac{d V}{d p}=-\frac{W}{p^{2}} \frac{d \rho}{d p}
$$

Substituting Eq.A-18 and Eq.A-19 in Eq.A-16 gives:

$$
c=-\frac{1}{\frac{W}{\rho}}\left(-\frac{W}{\rho^{2}} \frac{d \rho}{d p}\right)
$$

which reduces to:

$$
\begin{aligned}
& c=\frac{1}{\rho}\left(\frac{\partial \rho}{\partial p}\right)_{T} \\
& c p=\left(\frac{\partial \rho}{\partial p}\right)_{T}
\end{aligned}
$$

If we assume that the fluid is slightly compressible, and that the compressibility is constant, Eq.A-15 becomes:

$$
V \varphi \rho c \frac{d p}{d t}=w_{e}-w_{p}
$$

\section{A. 1.4. Hurst Simplified Solution}

Integrating Eq.A-22 between the limits of initial and final conditions gives:

$$
V \varphi \rho_{\alpha v} c \int_{p_{i}}^{p} d p=\int_{0}^{t} w_{e} d t-\int_{0}^{t} w_{e} d t
$$

If we define the volume of water $V_{w}=V_{\varphi}$, and assume constant rate, Eq. A-23 becomes:

$$
V_{w} \rho_{a v} c\left(p-p_{i}\right)=W_{a}-w_{p} \cdot t
$$

Define the pressure drop as $\Delta p=p_{i}-p$, then Eq.A-24 becomes: 


$$
-V_{w} \rho_{\alpha v} c \Delta p=W_{e}-w_{p} \cdot t
$$

The cumulative water influx is given by the convolution integral:

$$
W_{0}=B \int_{0}^{t_{D}} \frac{d \Delta p}{d t_{D}} Q_{D}\left(t_{D}-t_{D}\right) d t_{D}
$$

where $t_{D}$ is defined as:

$$
t_{D}=\frac{k t}{\varphi \mu c L^{2}}
$$

$L$ is a characteristic length, which is the length of the aquifer in the case of a linear finite system, and unit length in the case of an infinite system. Substituting Eq.A-26 and Eq.A-27 in Eq.A-25 gives:

$$
-V_{w} p_{\alpha v} c \Delta p=B \int_{0}^{t_{D}} \frac{d \Delta p}{d t_{D}} Q_{D}\left(t_{D}-t_{D}^{\dot{D}}\right) d t_{D}-\frac{\varphi \mu_{\alpha q} c_{\alpha q} L^{2} t_{D} w_{p}}{k_{\alpha q}}
$$

Taking the Laplace transform of Eq.A-28 with respect to $t_{D}$ gives:

$$
-V_{w} \rho_{\alpha \nu} c \Delta \bar{p}=B s \Delta \bar{p} \bar{Q}_{D}-\frac{\varphi \mu_{\alpha q} c_{a q} L^{2} w_{p}}{k_{\alpha q} s^{2}}
$$

Now the correct expressions for $\bar{Q}_{D}$ and $B$ must be used according to the boundary conditions and geometry. The solutions for $\bar{Q}_{D}$ are presented in Table 1 . The solution for the infinite linear case will now be presented.

$$
\begin{gathered}
\bar{Q}_{D}=s^{-\frac{\mathrm{s}}{2}} \\
B=A \varphi c_{\alpha q} \rho_{\alpha q}
\end{gathered}
$$

Substituting Eq.A-30 in Eq.A-29 gives ( $L$ is unit length):

$$
A \varphi c_{\alpha q} \rho_{\alpha q} s \Delta \bar{p} s{ }^{-\frac{s}{2}}+V_{w} \rho_{r e s} c_{r s s} \Delta \bar{p}=\frac{\varphi \mu_{\alpha q} c_{a q} w_{p}}{k_{\alpha q} s^{2}}
$$

Here the subscripts have been introduced to distinguish between the aquifer and the reservoir. Rearranging Eq.A-31 gives:

$$
\Delta \bar{p}\left(A \varphi c_{\alpha q} \rho_{\alpha q} s^{-1 / z}+V_{w} c_{r s s} \rho_{r s s}\right)=\frac{\varphi \mu_{\alpha q} c_{\alpha q} w_{p}}{k_{\alpha} s^{2}}
$$


- Now define $l$ as the length of the reservoir, then:

$$
V_{w}=V \varphi=A l \varphi
$$

where $A$ is the cross-sectional area of the aquifer and $l$ is the length of the reservoir. Substituting Eq.A-33 in Eq.A-32 and solving for $\Delta \bar{p}$ gives:

$$
\Delta \bar{p}=\frac{\varphi \mu_{a q} c_{\alpha q} w_{p}}{k_{\alpha q} s^{2}\left(A \varphi c_{\alpha q} \rho_{\alpha q} s^{-1 / 2}+A l \varphi c_{r a s} \rho_{r e s}\right)}
$$

We see that if the porosity of the aquifer and the reservoir are the same, it cancels from Eq.A-34. Rewriting Eq.A-34 gives:

$$
\Delta \bar{p}=\frac{\mu_{a q} c_{a q} w_{p}}{k_{a q} s^{\frac{3}{2}} A l c_{r e s} \rho_{r e s}\left(\frac{c_{a q} \rho_{a q}}{l c_{r e s} \rho_{r q s}}+s^{1 / 2}\right)}
$$

Now define a parameter $\lambda=\frac{c_{\text {eq }} \rho_{\text {aq }}}{l c_{\text {res }} \rho_{\text {res }}}$. Using this in Eq.A-35 gives:

$$
\Delta \bar{p}=\frac{\mu_{a q} c_{a q} w_{p}}{k_{a q} A l c_{r e s} \rho_{r e s}}\left[\frac{1}{s^{\frac{3}{2}}\left(\lambda+s^{1 / 2}\right)}\right]
$$

1. $\lambda$ very large:

We can ignore the term without $\lambda$, and Eq.A-36 becomes:

$$
\Delta \bar{p}=\frac{\mu_{a q} c_{a q} w_{p}}{k_{a q} A l c_{r s s} \rho_{\text {res }}}\left\lfloor\frac{1}{\lambda s^{\frac{g}{2}}}\right\rfloor
$$

Substituting the definition of $\lambda$ in Eq.A-37a gives:

$$
\Delta \bar{p}=\frac{\mu_{a q} w_{p}}{k_{a q} A \rho_{a q}}\left\lfloor\frac{1}{s^{\frac{g}{2}}}\right]
$$

The inverse transformation of Eq.A-37b is:

$$
\Delta p=\frac{\mu_{a q} w_{p}}{k_{\alpha q} A \rho_{a q}}\left[2{\frac{t_{D}}{\pi}}^{1 / 2}\right]
$$

which is the equation for an infinite linear aquifer. In the notation given by Nabor and Barham ${ }^{12}$, this becomes:

$$
\Delta p=\frac{\mu_{a q}}{k_{a q} A} q_{e} F_{1 / 2}
$$


and for variations in rate, by superposition:

$$
\Delta p=\frac{\mu_{a q}}{k_{a q} A_{a q}} \sum_{j=0}^{n} \Delta q_{j} F_{I / 2}\left(t_{D}-t_{D j}\right)
$$

2. $\lambda$ very small:

Now we ignore the term containing $\lambda$, and Eq.A-36 becomes:

$$
\Delta \bar{p}=\frac{\mu_{a q} c_{a q} w_{p}}{k_{\alpha q} A l c_{r a s} \rho_{r s s}}\left[\frac{1}{s^{2}}\right]
$$

The inverse transformation of Eq.A-39 is:

$$
\Delta p=\frac{\mu_{a q} c_{q a} w_{p} t_{D}}{k_{a q} A l c_{r e s} \rho_{\text {res }}}
$$

Substituting the definition of $t_{D}$ in Eq.A-40a gives:

$$
\Delta p=\frac{w_{p} t}{A l \varphi c_{\text {res }} p_{\text {res }}}
$$

which is the tank decompression of a confined system:

$$
\Delta p=\frac{1}{V \varphi c \rho} W_{p}
$$

3. $\lambda$ intermediate:

In this case the entire expression must be used. The inverse transformation of Eq.A-36 is given by:

$$
\Delta p=\frac{\mu_{\alpha q} c_{\alpha q} w_{p}}{k_{\alpha q} A l \rho_{\text {res }} c_{r e s} \lambda^{2}}\left[e^{\lambda^{\varepsilon_{t_{D}}}} \operatorname{erfc}\left(\lambda t_{b^{\prime 2}}\right)-1+\frac{2 \lambda t_{b^{\prime 2}}}{\pi^{1 / 2}}\right]
$$

By the superposition theorem, for varying rate we get:

$$
\Delta p=\frac{\mu_{a q} c_{\alpha q}}{k_{\alpha q} V_{r e s} \rho_{r e s} c_{r e s}} \sum_{j=0}^{n} \Delta w_{j} M\left[\lambda^{2}\left(t_{D}-t_{D j}\right)\right]
$$

where:

$$
M\left(\lambda^{2} t_{D}\right)=\frac{1}{\lambda^{2}}\left[e^{\lambda^{2} t_{D}} \operatorname{erfc}\left(\lambda t_{b^{\prime 2}}\right)-1+\frac{2 \lambda t_{D}^{\prime 2}}{\pi^{1 / 2}}\right]
$$




\section{A.2. Boiling Models}

\section{A.2. General Mass and Energy Balance}

In this case the energy balance must be included. The energy balance is as follows':

$$
m_{i} E_{i}-m_{c} E_{c}=Q_{i}^{\prime}-Q_{c}^{\prime}+h^{\prime}\left(m_{i}-m_{c}\right)
$$

where $m$ is the total mass of the system (fluid and rock). $h$ ' is the average enthalpy that accounts for the enthalpy change due to net mass change in the reservoir:

$$
h^{\prime}\left(m_{i}-m_{c}\right)=W_{p} h_{p}+W_{l} h_{l}-W_{e} h_{\varepsilon}
$$

The total energy change is given by the energy change in the fluid and the rock:

$$
m_{i} E_{i}-m_{c} E_{c}=W_{i} E_{i}-W_{c} E_{c}+V(1-\varphi) \rho_{\sigma} C_{\sigma}\left(T_{i}-T_{c}\right)
$$

and the net heat change transferred from the surroundings is:

$$
Q_{i}^{\prime}-Q_{c}^{\prime}=Q
$$

Substituting Eq.A-44, Eq.A-45, and Eq. 46 in Eq.A-43 gives:

$$
\begin{aligned}
& W_{i} E_{i}-W_{c} E_{c}+V(1-\varphi) \rho_{\sigma} C_{d}\left(T_{i}-T_{c}\right)= \\
& Q-W_{a} h_{a}+W_{p} h_{p}+W_{l} h_{l}
\end{aligned}
$$

We now introduce the mass balance:

$$
W_{c}=W_{i}+W_{\mathrm{o}}-W_{p}-W_{l}
$$

and the volumetric balance:

$$
V_{\varphi}=W_{i}\left[x_{i} \nu_{s i}+\left(1-x_{i}\right) v_{u i}\right]
$$

and the water influx is given by the VanEverdingen and Hurst superposition sum:

$$
W_{a}=B \sum_{j=0}^{n} Q_{D}\left(t_{D}-t_{D_{j}}\right) \Delta p_{j}
$$


Substituting Eq.A-48, Eq.A-49, and Eq.A-50 in Eq.A-47 and rearranging gives:

$$
\begin{aligned}
W_{p}\left(h_{p}-E_{c}\right)+ & W_{l}\left(h_{l}-E_{c}\right)+Q= \\
W\left[E_{i}-E_{c}\right. & \left.+\left(\frac{1-\varphi}{\varphi}\right)\left[x_{i} \nu_{s i}+\left(1-x_{i}\right) \nu_{w i}\right] \rho_{\sigma} C_{\sigma}\left(T_{i}-T_{c}\right)\right] \\
& +\left(h_{\varepsilon}-E_{c}\right) \cdot B \sum_{j=0}^{n} Q_{D}\left(t_{D}-t_{D j}\right) \Delta p_{j}
\end{aligned}
$$

\section{A2.2. Simplified Approach}

The total volume of the reservoir fluids is:

$$
V_{t}=V_{w}+V_{s}
$$

and the total mass of the reservoir fluids is:

$$
W_{t}=W_{w}+W_{s}
$$

The density of each phase is given by:

$$
\begin{gathered}
\rho_{w}=\frac{W_{w}}{V_{w}} \\
\rho_{s}=\frac{W_{s}}{V_{s}}
\end{gathered}
$$

The volume of each phase is given by:

$$
\begin{gathered}
V_{w}=S_{w} V_{t} \\
V_{s}=\left(1-S_{w}\right) V_{t}
\end{gathered}
$$

Substituting Eq.A-55 in Eq.A-52 gives:

$$
V_{t}=S_{w} V_{t}+\left(1-S_{w}\right) V_{t}
$$

The total effective density is:

$$
\rho_{t}=\frac{W_{t}}{V_{t}}
$$

From Eq.A-53, Eq.A-54, and Eq.A-55:

$$
W_{t}=\rho_{w} S_{w} V_{t}+\rho_{s}\left(1-S_{w}\right) V_{t}
$$

Substituting Eq.A-56, and Eq.A-58 in Eq.A-57 gives:

$$
\rho_{t}=\frac{\rho_{w} S_{w} V_{t}+\rho_{s}\left(1-S_{w}\right) V_{t}}{S_{w} V_{t}+\left(1-S_{w}\right) V_{t}}
$$


which reduces to:

$$
\rho_{t}=\rho_{w} S_{w}+\rho_{s}\left(1-S_{w}\right)
$$

Substituting Eq.A-60 in Eq.A-48 and assuming $W_{l}$ can be neglected, we get:

$$
V \varphi \rho_{t c}=V \varphi \rho_{t i}+W_{t}-W_{p}
$$

which rearranges to:

$$
V \varphi\left(\rho_{t c}-\rho_{t i}\right)=W_{e}-W_{p}
$$

\section{A.3. Total Compressibility}

The two phase compressibility is given by ${ }^{3 t}$ :

$$
c_{2 p h}=-\frac{1}{\varphi V} \frac{\Delta V}{\Delta p}=\frac{\left[(1-\varphi) \rho_{\sigma} C_{\sigma}+\varphi S_{w} \rho_{w} C_{w}\right]\left(\rho_{w}-\rho_{s}\right)}{\varphi L\left(d p_{s} / d T\right) \rho_{w} \rho_{s}}
$$

n Eq.A-63 $L$ is the latent heat of vaporization. Eq.A-63 should be used when liquid and steam are in equilibrium at saturated conditions. If this zone is only a part of the reservoir, the effect of the compressibility in this zone on the total system may be calculated using the definition of the compressibility:

$$
c_{t}=-\frac{1}{V_{t}}\left(\frac{\partial V_{t}}{\partial p}\right)
$$

where the total fluid volume is:

$$
V_{t}=V_{w}+V_{\text {2ph }}
$$

Differentiating Eq.A-65 with respect to $p$ gives:

$$
\frac{d V_{t}}{d p}=\frac{d V_{w}}{d p}+\frac{d V_{2 p h}}{d p}
$$

By the definition of $c_{w}$ and $c_{2 p h}$ :

$$
\begin{aligned}
c_{w} & =-\frac{1}{V_{w}}\left(\frac{\partial V_{w}}{\partial p}\right) \\
c_{2 p h} & =-\frac{1}{V_{2 p h}}\left(\frac{\partial V_{2 p h}}{\partial p}\right)
\end{aligned}
$$

Substituting Eq.A-67 in Eq.A-66 gives:

$$
\frac{d V_{t}}{d p}=-c_{w} V_{w}-c_{2 p h} V_{2 p h}
$$


Substituting Eq.A-65 and Eq.A-68 in Eq.A-64 gives:

$$
c_{t}=\frac{1}{V_{w}+V_{2 p h}}\left(c_{w} V_{w}+c_{2 p h} V_{2 p h}\right)
$$




\section{APPENDIX B: Data Files and Computer Programs.}

\section{B. 1. Data Files}

Thorhallsson ${ }^{30}$ provided the drawdown and mass flow-rate history for the first 2319 days of production at Svartsengi. The first data file "drawdwn", shows the number of days after production started in the first column and the measured drawdown in the second column. The first number is the number of data points.

The second data file "prodr", shows the number of days after production started in the first column and the total mass flow-rate in $\mathrm{kg} / \mathrm{s}$ from the reservoir in the second column. Each entry of rate in the file is effective from the time of the previous entry until the time corresponding to that entry. For example, between 388 and 419 days of production, the rate was $51 \mathrm{~kg} / \mathrm{s}$.

The third data file "input", shows the number of days after production started in the first column, the rate in $\mathrm{kg} / \mathrm{s}$ in the second column, and the measured drawdown in meters in the third column. Note that the drawdown was not always measured on the days when the rate was changed. For those cases a linear interpolation between the values in the file "drawdwn" gave the value for the drawdown in the flle "input". The flle "input" was used as the input file for all the programs. The cumulative mass produced was calculated using:

$$
W_{p_{n}}=W_{p_{n-1}}+w_{n}\left(\frac{k g}{s}\right)\left(t_{n}-t_{n-1}\right) \text { days } \cdot \frac{60 s}{\min } \cdot \frac{60 \mathrm{~min}}{h r} \cdot \frac{24 h r}{\text { days }}
$$

\section{B.2. The Computer Programs}

All the computer programs are written in fortran 77, and were run on the Stanford University Petroleum Engineering VAX 11/750 computer facility. The file "input" is used for input of time, rate, and measured drawdown. The following variable names are consistently used in the programs: 
$x=$ vector of length $124=$ time in days.

$\mathrm{y}=$ vector of length $124=$ rate in $\mathrm{kg} / \mathrm{s}$.

$\mathrm{dh}=$ vector of length $124=$ measured drawdown in meters.

dh1=calculated drawdown from the model in meters (a vector in some programs).

cum=vector of length $124=$ cumulative production in $\mathrm{kg}$.

For least squares fitting the subroutines "iflsq" and "llsqf" have been used. These subroutines are in the "imsl" library of subroutines. "iflsq" fits a user supplied function to a set of data using the least squares method. The program will determine the constants giving the best fit in an equation of the form:

$$
y_{i}=a_{1} f_{i, 1}+a_{2} f_{i, 2}+\cdots+a_{n} f_{i, n}
$$

where the function $f$ is a function of $x_{i}$ and $n$, and $(x, y)_{i}$ are the data points.

"llsqf" solves the set of equations shown in the section on history matching by minimizing the difference between the points which are given and the fitted points. 


$\begin{array}{ll}226 & \\ 1 \varnothing & 186 \\ 2 \varnothing & 1.88 \\ 3 \varnothing & 1.4 \varnothing \\ 4 \varnothing & 1.7 \varnothing \\ 5 \varnothing & 2.82 \\ 6 \varnothing & 2.14 \\ 7 \varnothing & 2.38 \\ 8 \varnothing & 2.58 \\ 9 \varnothing & 2.76 \\ 1 \varnothing \varnothing & 3.8 \varnothing \\ 11 \varnothing & 3.18 \\ 12 \varnothing & 3.32 \\ 13 \varnothing & 3.44 \\ 14 \varnothing & 3.94 \\ 15 \varnothing & 4.8 \varnothing \\ 16 \varnothing & 4.54 \\ 17 \varnothing & 4.54 \\ 18 \varnothing & 4.62 \\ 19 \varnothing & 4.76 \\ 2 \varnothing \varnothing & 5.8 \varnothing \\ 21 \varnothing & 6.8 \varnothing \\ 22 \varnothing & 4.54 \\ 23 \varnothing & 7.8 \varnothing \\ 24 \varnothing & 7.8 \varnothing \\ 25 \varnothing & 7.5 \varnothing \\ 26 \varnothing & 7.4 \varnothing \\ 27 \varnothing & 7.3 \varnothing \\ 28 \varnothing & 7.3 \varnothing \\ 29 \varnothing & 7.5 \varnothing \\ 3 \varnothing \varnothing & 7.7 \varnothing \\ 31 \varnothing & 7.8 \varnothing \\ 32 \varnothing & 8.9 \varnothing \\ 33 \varnothing & 8.1 \varnothing \\ 34 \varnothing & 8.3 \varnothing \\ 35 \varnothing & 8.38 \\ 36 \varnothing & 8.46 \\ 37 \varnothing & 8.54 \\ 38 \varnothing & 8.62 \\ 39 \varnothing & 8.7 \varnothing \\ 4 \varnothing \varnothing & 9.28 \\ 41 \varnothing & 9.86 \\ 42 \varnothing & 10.44 \\ 43 \varnothing & 11.82 \\ 44 \varnothing & 11.6 \varnothing \\ 45 \varnothing & 11.9 \varnothing \\ 46 \varnothing & 12.1 \varnothing \\ 47 \varnothing & 12.4 \varnothing \\ 48 \varnothing & 12.7 \varnothing \\ 49 \varnothing & 12.9 \varnothing \\ 5 \varnothing \varnothing & 13.1 \varnothing \\ 51 \varnothing & 13.3 \varnothing \\ 52 \varnothing & 13.6 \varnothing \\ 53 \varnothing & 13.8 \varnothing \\ 54 \varnothing & 13.9 \varnothing \\ 55 \varnothing & 13.7 \varnothing \\ 56 \varnothing & 13.5 \varnothing \\ 57 \varnothing & 13.5 \varnothing \\ 576 & 13.5 \varnothing \\ 58 \varnothing & 13.8 \varnothing \\ 59 \varnothing & 14.2 \varnothing \\ 6 \varnothing \varnothing & 14.5 \varnothing \\ 61 \varnothing & 14.7 \varnothing \\ 62 \varnothing & 15.8 \varnothing\end{array}$

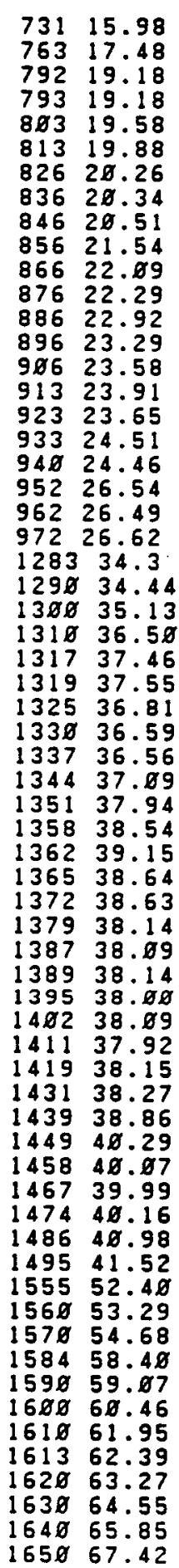

$1660 \quad 68.44$

167069.33

$1680 \quad 69.87$

169869.43

$1700 \quad 70.29$

171069.40

172868.98

173068.69

$1748 \quad 68.49$

$1750 \quad 68.27$

176068.16

177868.59

178069.20

179069.44

180072.18

181073.77

182875.88

184077.32

185678.34

186079.34

187879.64

188080.73

189081.68

$1908 \quad 82.41$

191883.17

192083.96

$1930 \quad 84.64$

194085.10

195085.32

196885.83

197486.60

$1978 \quad 86.66$

198386.76

198986.72

199186.79

199586.94

200087.14

$\begin{array}{ll}2008 & 87.27\end{array}$

$2053 \quad 89.63$

$2056 \quad 89.78$

206189.86

206690.81

207190.18

$2076 \quad 90.33$

208190.42

$2086 \quad 90.44$

209190.56

$2096 \quad 90.67$

218190.66

$2106 \quad 90.91$

211191.03

211591.87

213091.87

213292.13

$2137 \quad 92.48$

214392.90

$2148 \quad 93.54$

215393.75

$2158 \quad 94.09$

$2164 \quad 93.88$

$2178-11.58$

$2183 \quad 37.61$

$2185 \quad 45.71$

$2186 \quad 51.05$
$2188 \quad 64: 20$

$2189 \quad 67.96$

219078.67

219175.01

219278.50

$2193 \quad 82.09$

$2194 \quad 85.27$

$2196 \quad 89.45$

$2198 \quad 90.86$

219991.89

220092.99

228193.95

220295.13

$2203 \quad 96.31$

$2204 \quad 96.35$

221093.14

222094.60

222795.27

223496.08

224196.67

$2248 \quad 97.36$

226198.58

$2270 \quad 99.32$

2280100.38

2288101.59

2290101.76

2296102.12

$23.02 \quad 102.58$

2310102.96

2319103.38 
prodr

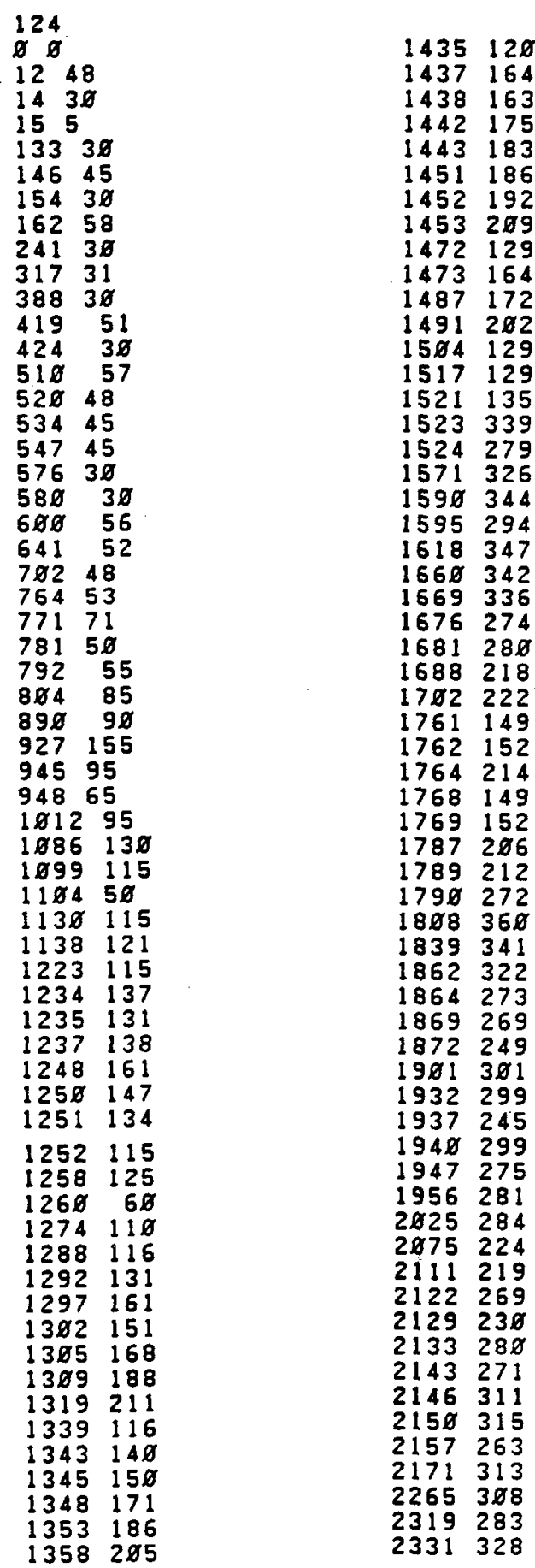


input

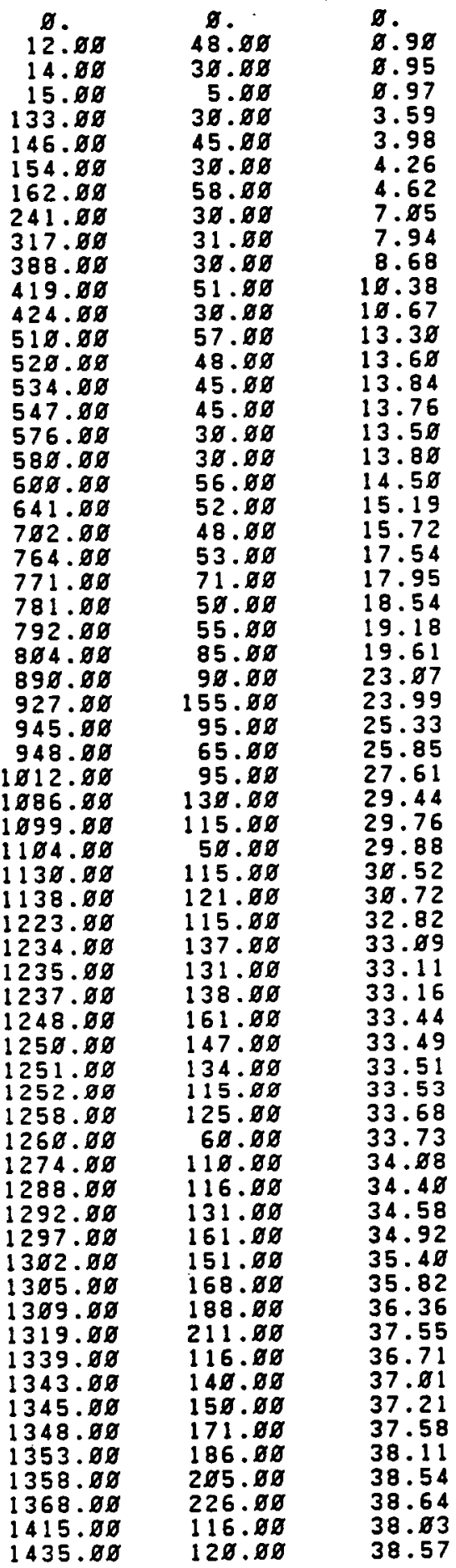

\begin{tabular}{|c|c|c|}
\hline 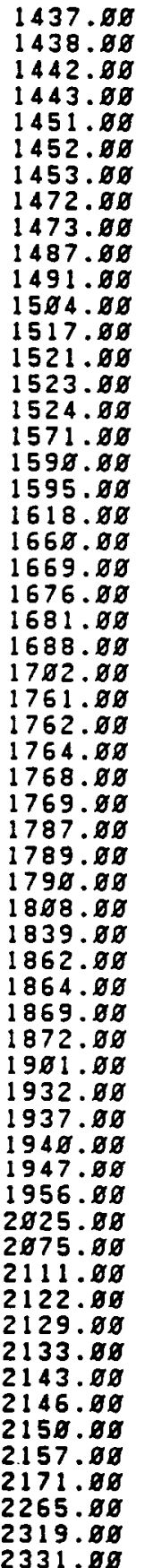 & 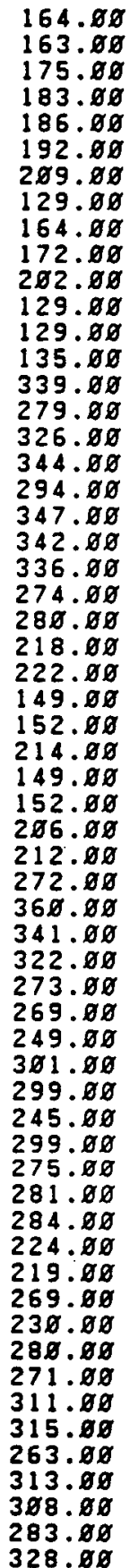 & $\begin{array}{l}38.71 \\
38.79 \\
39.29 \\
39.43 \\
48.24 \\
48.22 \\
48.19 \\
40.11 \\
48.14 \\
41.84 \\
41.28 \\
43.15 \\
45.51 \\
46.23 \\
46.68 \\
46.78 \\
54.95 \\
59.87 \\
59.76 \\
63.82 \\
68.44 \\
69.24 \\
69.65 \\
69.83 \\
69.52 \\
78.11 \\
68.28 \\
68.25 \\
68.33 \\
68.58 \\
68.55 \\
69.37 \\
69.42 \\
69.44 \\
73.44 \\
77.21 \\
79.48 \\
79.46 \\
79.61 \\
79.86 \\
82.49 \\
84.73 \\
84.96 \\
85.10 \\
85.25 \\
85.63 \\
88.16 \\
90.38 \\
91.83 \\
91.44 \\
91.82 \\
92.28 \\
92.98 \\
93.28 \\
93.62 \\
94.82 \\
41.15 \\
98.91 \\
183.38 \\
183.83\end{array}$ \\
\hline
\end{tabular}




\section{The bolling model for suartsengl}

This program determines the constants $C 1$ and $C 2$ using the least squares method.

The subroutine llsqf must be supplied by the user.

The drawdown is then calculated using the fitted constants.

Implicit real*4(a-h,o-z)

dimension $x(124), y(124), d h(124)$, cum(124), sum(124)

rea 1 mat $(123,2), a(2), h(2), b(123)$, to

integer la,m,n,kbasis, ip (2), ler

write $(6, *) 123$

do 1 i $=1,123$

$\operatorname{read}(5, *) \times(1), y(1), d h(1)$

cum $(i)=c u m(i-i)+(x i)-x(i-1)) * y(i) * 60 . * 60 . * 24$.

do $5 j=1,1$

$W P=\varnothing$.

$k=1$

$x 1=x(1)-x(j)$

10

If $(x) \cdot g=. x(k))$ then

$w p=w p+i x(k)-x(k-1)) * y(k) * 6 \varnothing . * 6 \varnothing . * 24$.

$k=k+1$

go to 10

eise

$w p=w p+(x 1-x(k-1)) * y(k) * 6 \varnothing . * 6 \pi . * 24$.

$$
\text { endif }
$$

$\operatorname{sum}(1)=\operatorname{sum}(1)+w p * \exp (-x(j) / 250) *.(x(j)-x(j-1))$

5

cont inue

write $\left(6^{*}\right) \operatorname{cum}(i)$, oh $(1)$

$\operatorname{mat}(1,1)=\operatorname{cum}(1)$

$\operatorname{mat}(1,2)=\operatorname{sum}(1)$

$b(1)=d h(1)$

1 continue

$1 a=123$

$m=123$

$n=2$

tol $=0.0$

kbas $1 \mathrm{~s}=2$

call lisqfimat, ia,m,n,oh,tol,kbasis,a,h,fp,ier)

write $(6, *) a(1), a(2)$

write $(6, *) 123$

do $2 \quad i=1,123$

$d h l=a(1) * \operatorname{cum}(1)+a(2) * \operatorname{sum}(1)$

2

write $(6, *) \in u m(t)$, dh 1

cont inue

stop

end 

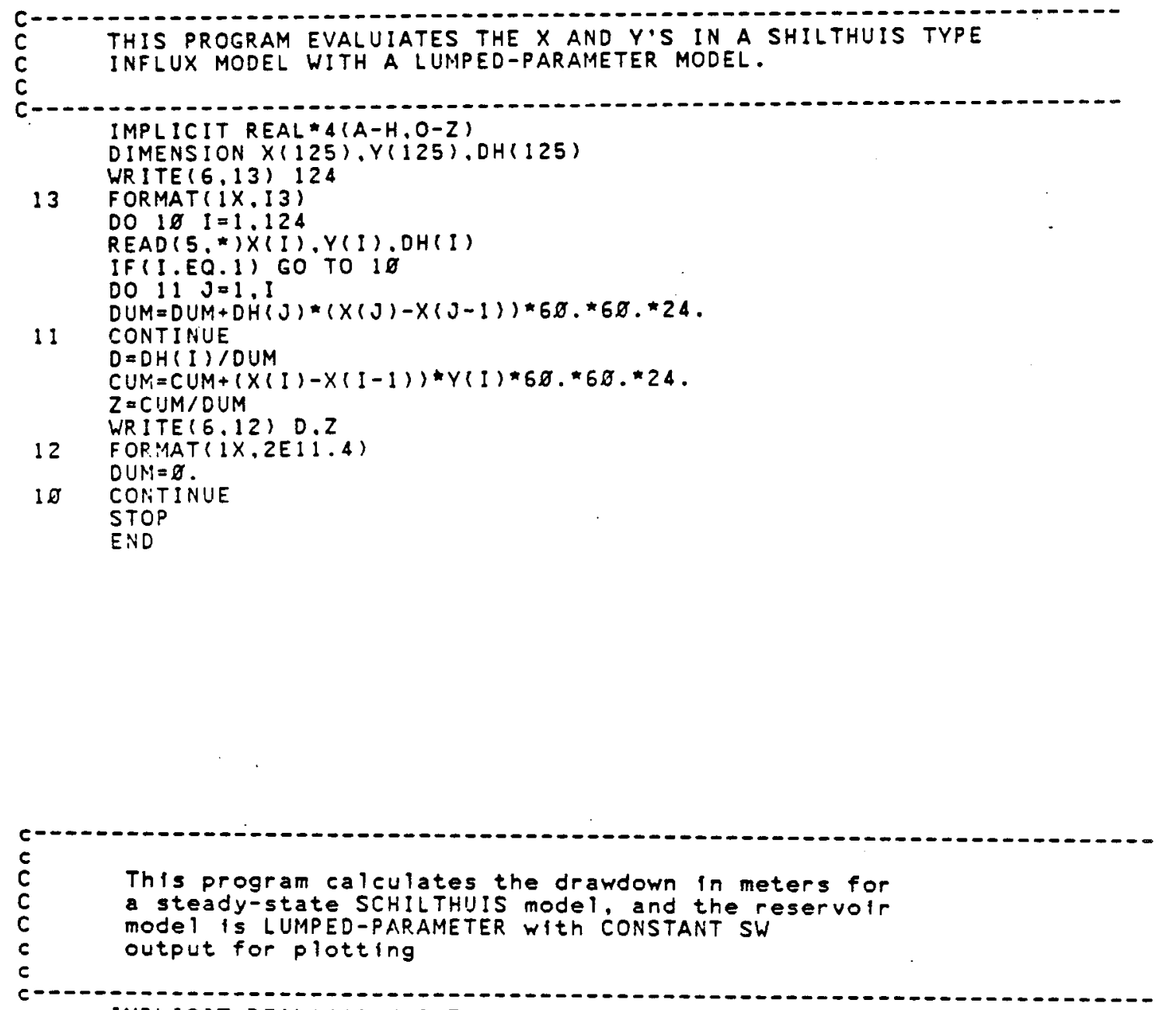

IMPLICIT REAL*4(A-H, O-Z)

DIMENSION $X(125), \mathrm{CUm}(125), Y(125), \mathrm{D}(125), \mathrm{DH}(125)$

$A !=2.75447$

$A \varnothing=6.60567 e 7$

13 format $(i x, 13)$

DO $1.8 I=1.124$

READ (5.*)X(I),YII),DH(I)

IF (I.EO.1) GO TO 20

SUM $=S U M+O(I-1) *(X(I-1)-X(1-2))=60 . \approx 60 . * 24$.

$\operatorname{CUM}(1)=\operatorname{CUM}(1-1)+(X(1)-X(1-1)) * Y(1) * 60 . * 60 . * 24$.

20 WRITE $(6, *) \operatorname{cum}(1), D(I)$

IO CONTINUE

wr t te $(6,13) 124$

do $30 \quad 1=1.124$

30 continue

END 


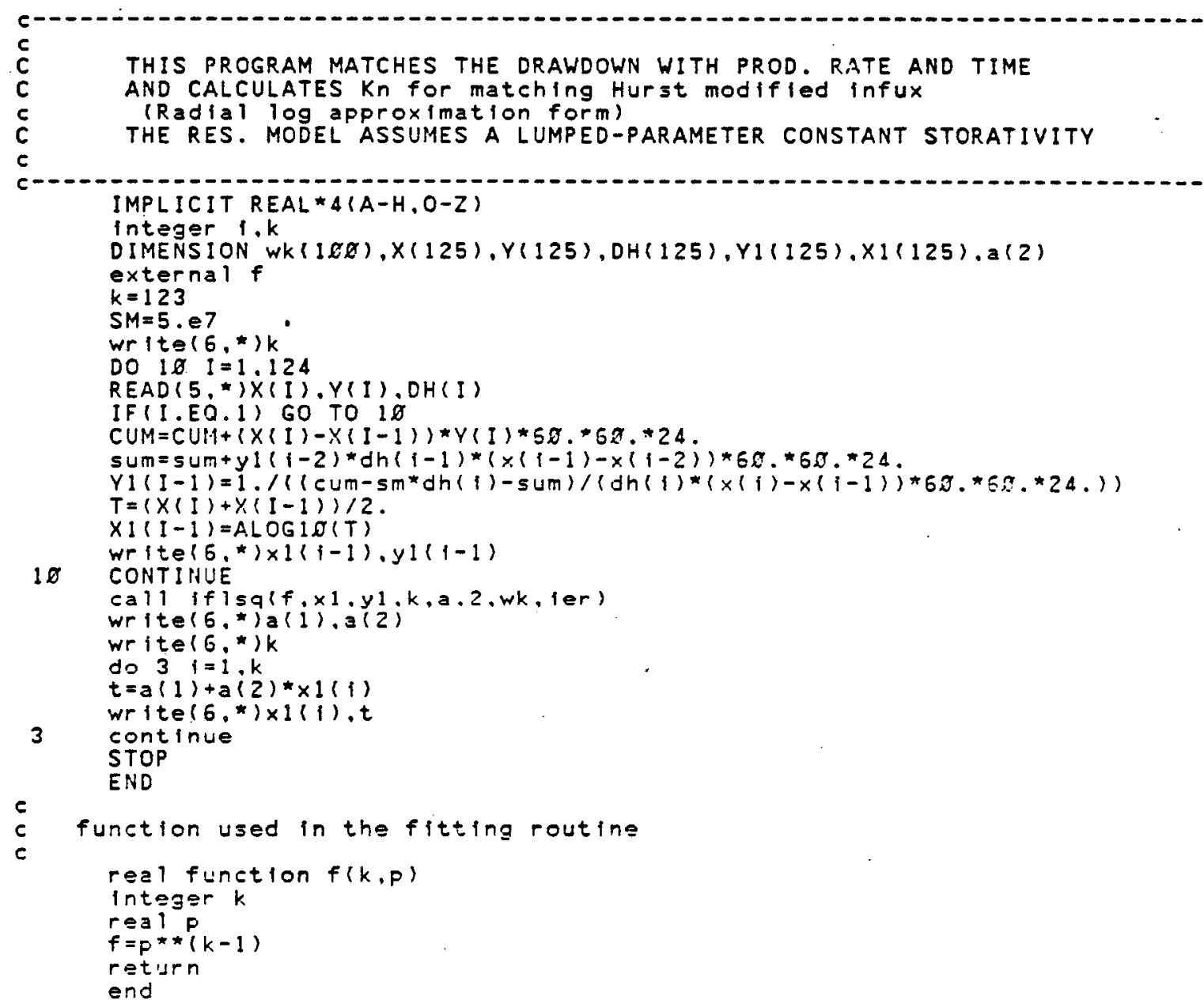




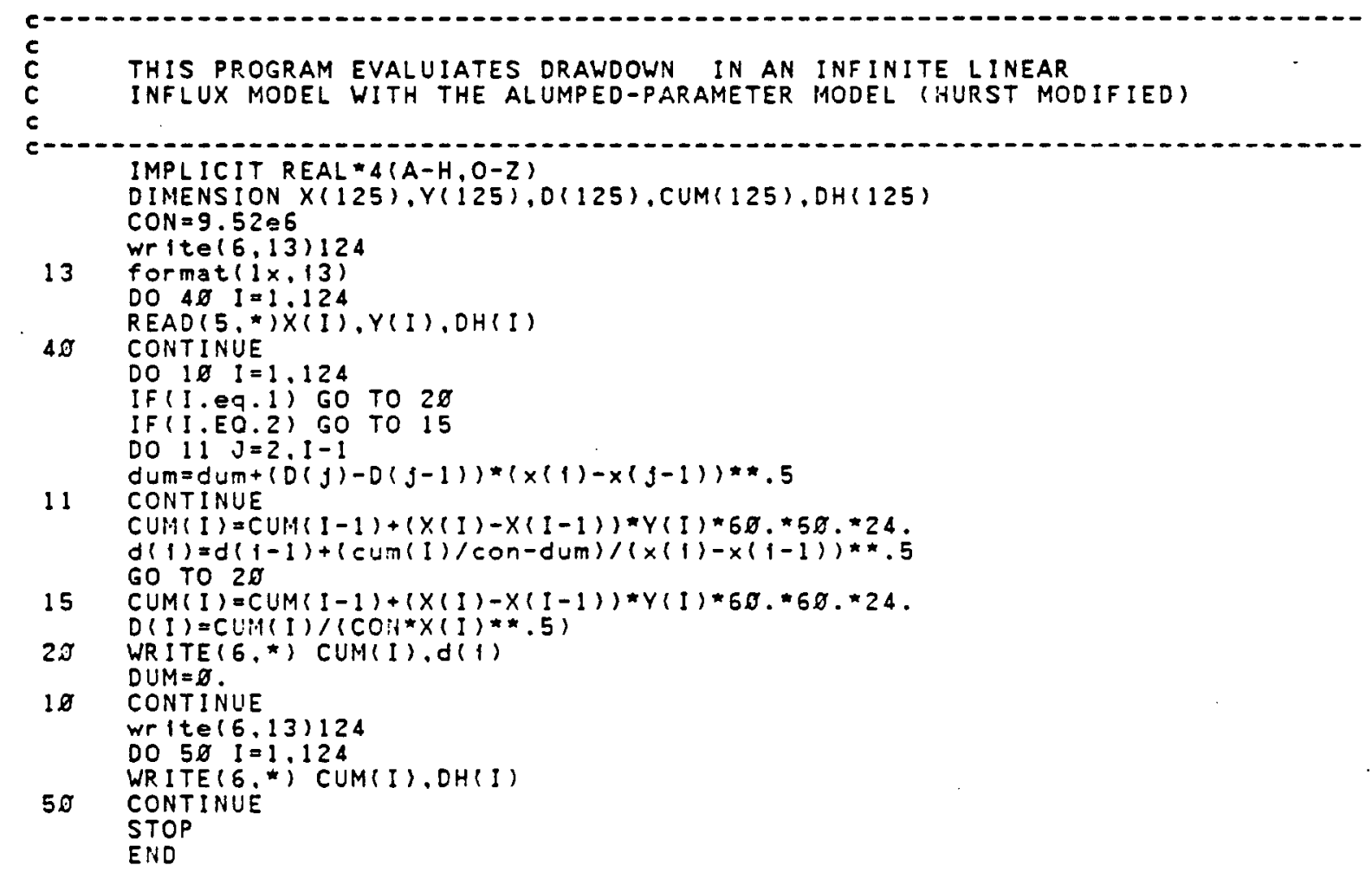




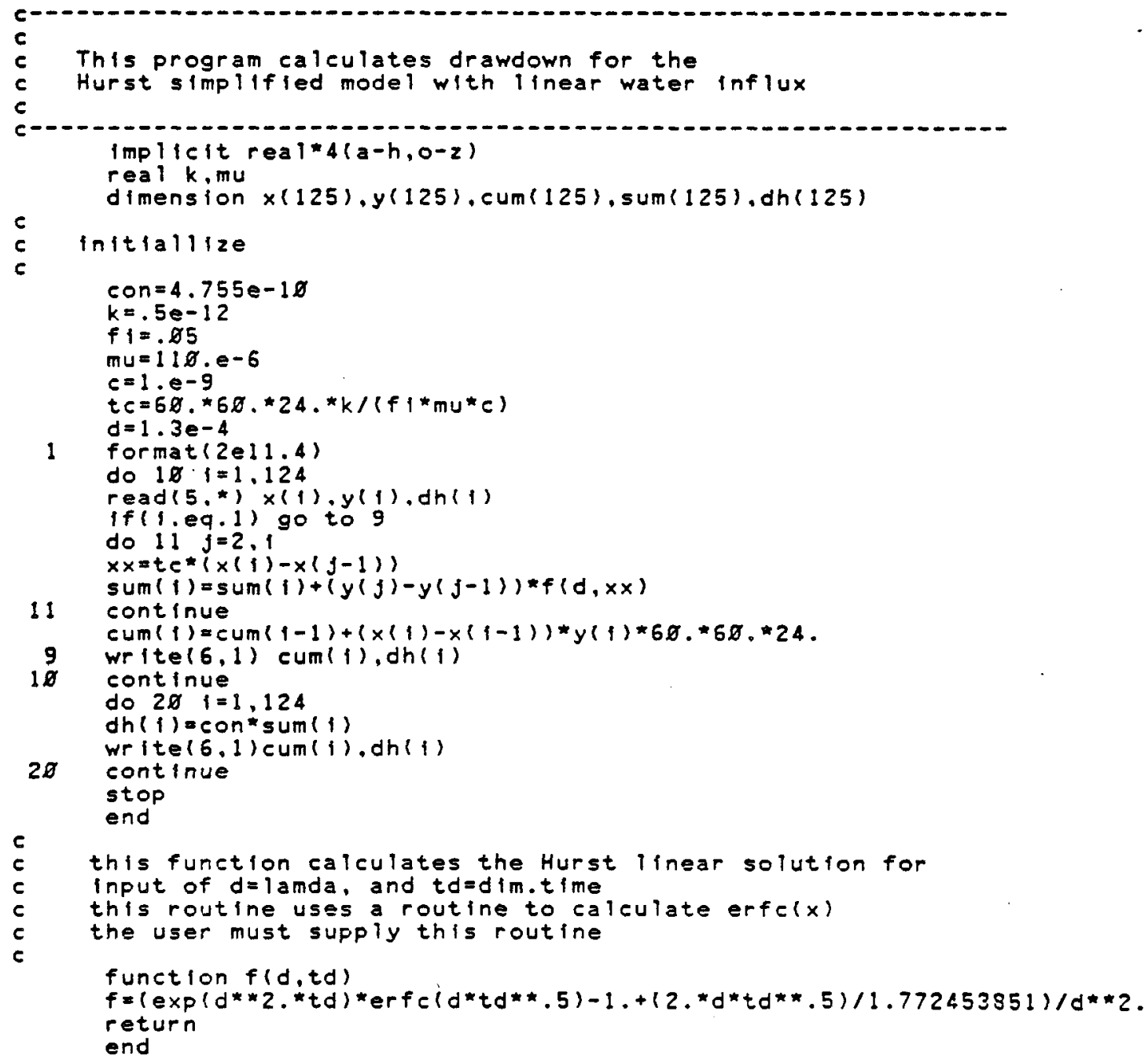




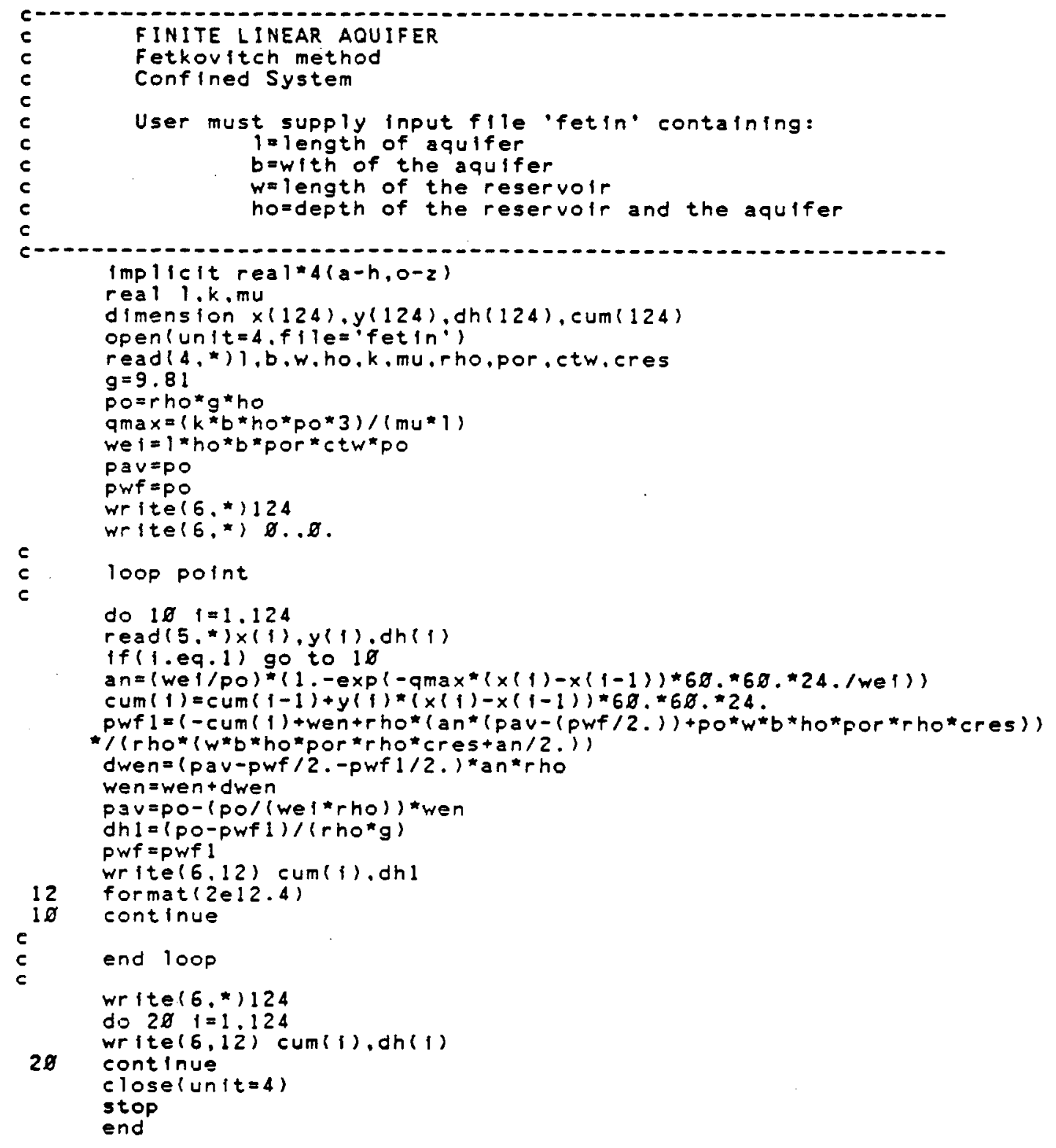




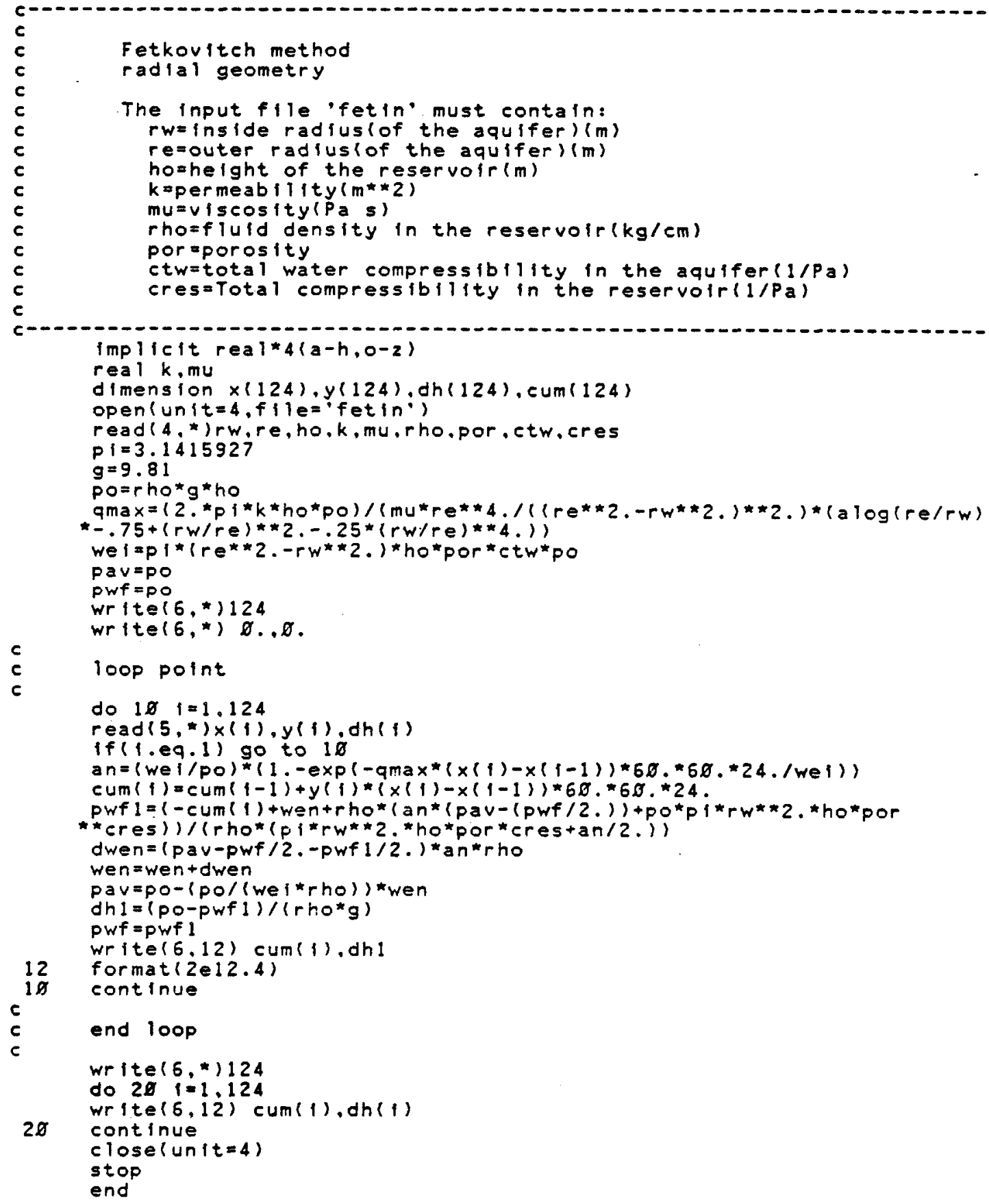




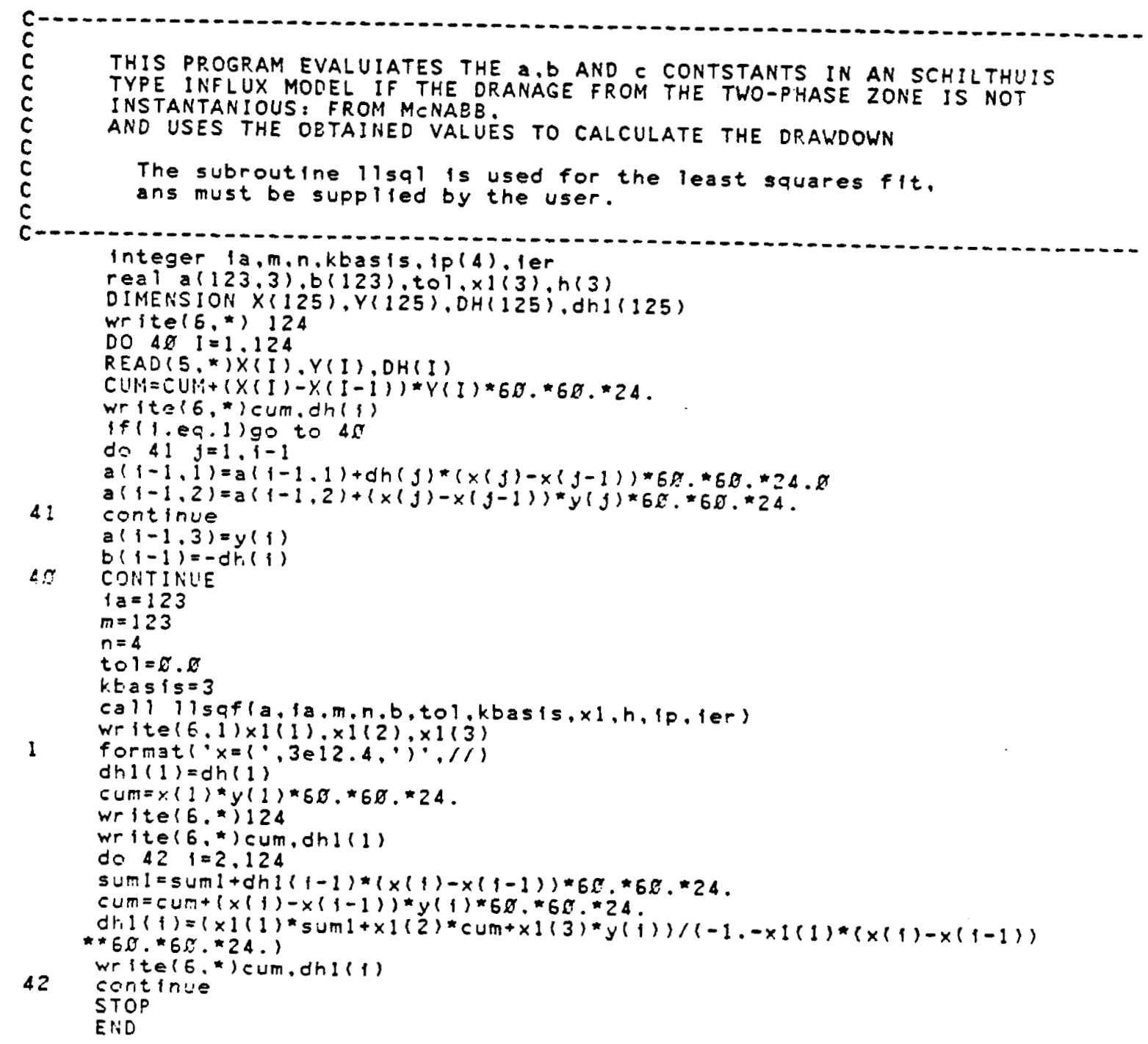

